\title{
Toric morphisms and fibrations of
}

\section{toric Calabi-Yau hypersurfaces}

\author{
Yi Hu${ }^{1}$, Chien-Hao Liu ${ }^{2}$, and Shing-Tung $\mathbf{Y a u}^{3}$ \\ ${ }^{1}$ Department of Mathematics, University of Arizona, Tucson, Arizona 85721 \\ yhu@math.arizona.edu \\ ${ }^{2}$ Department of Mathematics, Harvard University, Cambridge, MA 02138 \\ chienliu@math.harvard.edu \\ ${ }^{3}$ Department of Mathematics, Harvard University, Cambridge, MA 02138 \\ yau@math.harvard.edu
}

\begin{abstract}
Special fibrations of toric varieties have been used by physicists, e.g. the school of Candelas, to construct dual pairs in the study of Het/F-theory duality. Motivated by this, we investigate in this paper the details of toric morphisms between toric varieties. In particular, a complete toric description of fibers - both generic and non-generic -, image, and the flattening stratification of a toric morphism are given. Two examples are provided to illustrate the discussions. We then turn to the study of the restriction of a toric morphism to a toric hypersurface. The details of this can be understood by the various restrictions of a line bundle with a section that defines the hypersurface. These general toric geometry discussions give rise to a computational scheme for the details of a toric morphism and the induced fibration of toric hypersurfaces therein. We apply this scheme to study the family of complex 4-dimensional elliptic Calabi-Yau toric hypersurfaces that appear in a recent work of Braun-Candelas-dlOssa-Grassi. Some directions for future work are listed in the end.
\end{abstract}

Key words: heterotic-string/F-theory duality, toric morphism, fibration, primitive cone, relative star, toric Calabi-Yau hypersurface, fibred Calabi-Yau manifold, elliptic Calabi-Yau manifold.

MSC number 2000: 14M25, 81T30, 14J32, 14D06, 14J35.

e-print archive: http://xxx.lanl.gov/math.AG/0010082 
Acknowledgements. This work is a further study of some original ideas from Philip Candelas and his school. We would like to thank him for educations and the source of inspirations. We would like to thank also Alex Avram, Volker Braun, Hung-Wen Chang, Jiun-Cheng Chen, Ti-Ming Chiang, Xenia de la Ossa, Jacques Distler, Daniel Freed, Robert Gompf, Antonella Grassi, Brian Greene, Joe Harris, Mark Haskins, Shinobu Hosono, Sheldon Katz, Sean Keel, Albrecht Klemm, Bong H. Lian, Chiu-Chu Liu, David Morrison, Eugene Perevalov, Govindan Rajesh, Lorenzo Sadun, David Saltman, Herald Skarke, Jason Starr, Margaret Symington, Richard Thomas, Karen Uhlenbeck, Cumrun Vafa, Xiao-Wei Wang, and Eric Zaslow for valuable inspirations, educations, conversations, and correspondences at various stages of the work. C.H.L. would like to thank in addition Orlando Alvarez and William Thurston for indispensable educations, P.C. for the two years' group meetings in his school, the Geometry and String Theory Group at University of Texas at Austin for crucial influences at the brewing stage of the work, Nathan Dunfield for making the Adobe figures latex compatible, and Ling-Miao Chou for tremendous moral support. The work is supported by DOE grant DE-FG02-88ER25065 andiNSF grant DMS-9803347.

\section{Introduction and outline.}

\section{Introduction.}

Special fibrations of toric varieties have been used by physicists, e.g. the school of Candelas (cf. [A-K-M-S], [B-C-dlO-G], [B-M], [C-F], [C-P-R], [K-S1], [K-S2], [P-S], and $[\mathrm{Ra}]$ ), to construct dual pairs in the study of Heterotic-string/F-theory duality. This motivates us to investigate the details of toric morphisms between toric varieties and the induced morphisms from the toric hypersurfaces in the domain toric variety of a toric morphism to the target toric variety. The goal of this paper is to describe these details in terms of toric geometry and then to provide a computational scheme for actual toric computations.

The toric description of the generic fiber of a toric morphism has been discussed extensively e.g. in the works of the school of Candelas. Thus, the first theme of this article is to extend their results to a more general setting that takes care of all the fibers - generic and non-generic alike - in terms of toric geometry. The concept of primitive cones and relative stars with respect to a toric morphism are introduced along the way.

Once the details of a toric morphism are described in terms of toric geometry, our second theme is to understand the details of the induced morphism from a toric hypersurface in the domain toric variety to the target toric variety. This is an important step in understanding fibred toric Calabi-Yau hypersurfaces. It turns out that, by turning the problem to a study of the various restrictions of the line bundle with a section that defines the hypersurface, one can extract certain details of this induced morphism in terms of toric geometry as well.

As long as applications to Heterotic-string/F-theory duality is concerned, the discussions in this article will be of no use if no methods of concrete toric computa- 
tions for the various details outlined above can be provided. Thus our third theme in this article is to give a computational scheme to match with the toric geometry discussion. This is demonstrated by working out more details of an example: the toric morphism from a toric 5 -variety to a toric 3 -variety and the induced elliptic fibration of a 4-dimensional toric Calabi-Yau hypersurfaces from a recent collaborative work of Braun, Candelas, de la Ossa, and Grassi ([B-C-dlO-G]). We expect that the computational scheme presented here together with other works from the school of Candelas, notably the classification by Kreuzer and Skarke of higher dimensional reflexive polytopes that admit fibrations (e.g. [K-S1] and [K-S2]), will provide us with some more concrete understanding of fibred Calabi-Yau manifolds beyond current theory. This will await another work in future.

This article is organized as follows. After giving the references of the necessary backgrounds for physicists in Sec. 1, we study in Sec. 2 the various details of a toric morphism. In particular, a complete toric description of fibers - both generic and non-generic -, image, and the flattening stratification of a toric morphism are worked out in Sec. 2.1. Two examples are provided in Sec. 2.2 to illustrate the discussions. We then turn to the study of the restriction of a toric morphism to a toric hypersurface. After providing some necessary facts about line bundles on a toric variety in Sec. 3.1, we relate in Sec. 3.2 the problem of induced morphism to the equivalent problem of the various restrictions of a line bundle and the section in it that defines the hypersurface and study the latter. These general toric geometry discussions give rise to a computational scheme for the details of a toric morphism and the induced morphism of toric hypersurfaces therein. After recalling some necessary facts from the work of Batyrev in Sec. 4.1, we lay down this scheme in Sec. 4.2 and apply it to study the family of 4-dimensional elliptic Calabi-Yau toric hypersurfaces that was discussed in a recent work of Braun-Candelas-dlOssaGrassi. The Maple codes that are employed for the computation can be found in the preprint version of this article: math.AG/0010082. Some directions for future work are listed in Sec. 5.

Convention 0.1 [real vs. complex manifolds]. A real $n$-dimensional manifold will be called an $n$-manifold while a complex $n$-dimensional manifold an $n$-fold. Also, a real $n$-dimensional orbifold will be called an $n$-orbifold and a complex $n$ dimensioanl variety will be called an $n$-variety. All the varieties in this article are over $\mathbb{C}$ and by a point in a variety we mean a closed point of that variety only.

Convention 0.2 [morphism vs. fibrations]. In this article, a morphism $f$ : $X \rightarrow Y$ means a general map between varieties $X$ and $Y$ as in algebraic geometry; a fiber of $f$ means the point-set pre-image $f^{-1}(y)$ of $f$ at a certain (closed) point $y \in Y$. A morphism $f: X \rightarrow Y$ is called a fibration if $f$ is surjective and all its fibers have the same dimension $\operatorname{dim} X-\operatorname{dim} Y$.

\section{Outline.}

1. Essential mathematical background for physicists.

2. The toric geometry of toric morphisms. 
2.1 The toric geometry of toric morphisms.

2.2 Examples of toric morphisms and their fibers.

3. Induced morphism and fibers for hypersurfaces.

3.1 Preparations.

3.2 The induced morphism: $Y \subset X_{\Sigma^{\prime}} \rightarrow X_{\Sigma}$.

4. Fibration of Calabi-Yau hypersurfaces via toric morphisms.

4.1 General remarks.

4.2 The computational scheme and a detailed study of an example.

5. Remarks on further study.

\section{Essential mathematical background for physi- cists.}

Some references of the necessary mathematical backgrounds are provided here for the convenience of physicists. The notations of the basic ingredients of toric geometry are also introduced in this section. Essential necessary facts will be recalled along the way of discussions.

- Toric geometry. Excellent expositories are given in [C-K], [Fu], and [Gr]. See also [Da], [Do], [Ew], [G-K-Z3], [Od2], and [Zi]. In this article, we fix the following notations :

Notation:

$N \cong \mathbb{Z}^{n}:$ a lattice;

$M=\operatorname{Hom}(N, \mathbb{Z}):$ the dual lattice of $N$;

$T_{N}=\operatorname{Hom}\left(M, \mathbb{C}^{*}\right):$ the (complex) torus associated to $N$;

$\Sigma$ : a fan in $N_{\mathbb{R}}$

$\tau \prec \sigma$ : the relation between cones $\tau$ and $\sigma$ that $\tau$ is in the face of $\sigma$;

$X_{\Sigma}$ : the toric variety associated to $\Sigma$;

$\Sigma(i)$ : the $i$-skeleton of $\Sigma$;

$U_{\sigma}$ : the local affine chart of $X_{\Sigma}$ associated to $\sigma$ in $\Sigma$;

$x_{\sigma} \in U_{\sigma}$ : the distinguished point associated to $\sigma$;

$O_{\sigma}$ : the $T_{N}$-orbit of $x_{\sigma}$ under the $T_{N}$-action on $X_{\Sigma}$;

$V(\sigma)$ : the orbit closure of $O_{\sigma}$;

$N_{\sigma}$ : the sublattice of $N$ generated as a subgroup by $\sigma \cap N$;

$M(\sigma)=\sigma^{\perp} \cap M$; 
$\operatorname{Span}_{\mathbb{R}}(\sigma)$ : the real vector subspace spanned by $\sigma ;$

interior of $\sigma$ : the topological interior of $\sigma$ in $\operatorname{Span}_{\mathbb{R}}(\sigma)$;

$\lambda_{v}$ : the one-parameter subgroup of $T_{N}$ associated to $v \in N$;

$\chi^{m}$ : the character of $T_{N}$ associated to $m \in M$.

- Cox homogeneous coordinate ring. See [C-K] and [Cox].

- Toric Calabi-Yau hypersurfaces. See [Ba2]. Also [Bo], [B-B], [C-K], and [Hü].

- Fibred Calabi-Yau spaces. See [Og1], [Og2], and [Og3]. Also [A-M], [H-L$\mathrm{Y}],[\mathrm{K}-\mathrm{L}-\mathrm{M}],[\mathrm{K}-\mathrm{L}-\mathrm{R}-\mathrm{Y}]$ and the various works from the school of Candelas, e.g. [A-K-M-S], [B-C-dlO-G], [B-M], [C-F], [C-P-R], [K-S1], [K-S2], [P-S], and [Ra].

\section{The toric geometry of toric morphisms.}

The toric description of details of a toric morphism is given in Sec. 2.1. Two examples are provided in Sec. 2.2 to illustrate the ideas.

\subsection{The toric geometry of toric morphisms.}

Recall first the definition:

Definition 2.1.1 [toric morphism]. ([Ew] and [Fu].) Let $\Sigma^{\prime}, \Sigma$ be fans in $N^{\prime}$, $N$ respectively. A map between fans, in notation $\varphi: \Sigma^{\prime} \rightarrow \Sigma$, is a homomorphism $\varphi: N^{\prime} \rightarrow N$ of lattices that satisfies the condition: For each $\sigma^{\prime} \in \Sigma^{\prime}$, there exists a $\sigma \in \Sigma$ such that $\varphi\left(\sigma^{\prime}\right) \subset \sigma$. Such $\varphi$ determines a morphism $\tilde{\varphi}: X_{\Sigma^{\prime}} \rightarrow X_{\Sigma}$. A morphism between toric varieties that arises in this way is called a toric morphism. (Cf. Figure 2-1-1.)

The following two facts, adapted from [Ew] and [Ei], are fundamental to understanding toric morphisms.

Fact 2.1.2 [equivariance]. (Cf. Theorem 6.4 in [Ew].) The toric morphism $\tilde{\varphi}$ : $X_{\Sigma^{\prime}} \rightarrow X_{\Sigma}$ is equivariant with respect to the homomorphism $T_{N^{\prime}} \rightarrow T_{N}$ induced by $\varphi: N^{\prime} \rightarrow N$.

Fact 2.1.3 [semicontinuity of fiber dimension]. (Cf. Theorem 14.8 in [Ei].) Let $\varphi: X \rightarrow Y$ be a morphism between projective varieties and $s=\max \{\operatorname{dim} X-$ 


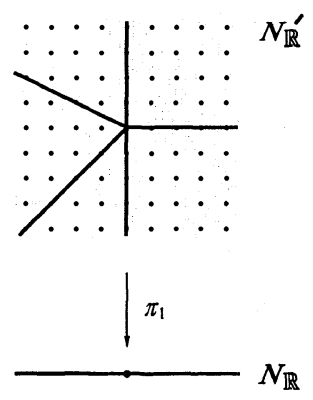

(a)

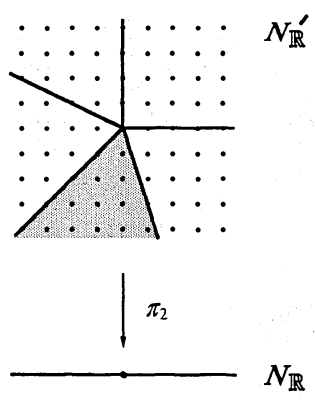

(b)

Figure 1: Figure 2-1-1. Both $\pi_{1}$ and $\pi_{2}$ are the natural projection map. In (a) $\pi_{1}$ induces a map between fans while in (b) $\pi_{2}$ does not. The cone of the fan in (b) that is not compatible with $\pi_{2}$ is indicated by a darker shading.

$\operatorname{dim} Y, 0\}$. Then there is a finite filtration of $Y$ by subvarieties (which need not be all distinct),

$$
Y \supset \varphi(X)=Y_{s} \supset Y_{s+1} \supset \cdots \supset Y_{\text {dim } X},
$$

such that $\operatorname{dim} \varphi^{-1}(y) \geq i$ if and only if $y \in Y_{i}$.

The main goal of this subsection is to prove Proposition 2.1.4 below, which extends both a result in [A-K-M-S] related to reflexive polytopes and the usual description of toric bundles as in e.g. [Ew] and [Fu] to full generality. It contains the toric analogue of Fact 2.1.3.

Toric morphism : Image, fibers, and a flattening stratification.

Proposition 2.1.4 [toric morphism]. Let $\tilde{\varphi}: X_{\Sigma^{\prime}} \rightarrow X_{\Sigma}$ be a toric morphism induced by a map of fans $\varphi: \Sigma^{\prime} \rightarrow \Sigma$. Then:

- The image $\widetilde{\varphi}\left(X_{\Sigma^{\prime}}\right)$ of $\tilde{\varphi}$ is a subvariety of $X_{\Sigma}$. It is realized as the toric variety associated to the fan $\Sigma_{\varphi} \doteq \Sigma \cap \varphi\left(N_{\mathbb{R}}^{\prime}\right)$.

- The fiber of $\widetilde{\varphi}$ over a point $y \in X_{\Sigma_{\varphi}}$ depends only on the orbit $O_{\sigma}, \sigma \in \Sigma_{\varphi}$, that contains $y$. Denote this fiber by $F_{\sigma}$, then it can be described as follows.

Define $\Sigma_{\sigma}^{\prime}$ to be the set of cones $\sigma^{\prime}$ in $\Sigma^{\prime}$, whose interior is mapped to the interior of $\sigma$. Let Ind $(\sigma)$ be the index of $\tilde{\varphi}$ over $O_{\sigma}$ (cf. Definition/Lemma 2.1.7). Then $\varphi^{-1}(y)=F_{\sigma}$ is a disjoint union of Ind $(\sigma)$ identical copies of connected reducible toric variety $F_{\sigma}^{c}$, whose irreducible components $F_{\sigma}^{\tau^{\prime}}$ are the toric variety associated to the relative star $\operatorname{Star}_{\sigma}\left(\tau^{\prime}\right)$ of the primitive elements $\tau^{\prime}$ in $\Sigma_{\sigma}^{\prime}$ (cf. Definition 2.1.9, Definition 2.1.10, and Lemma 2.1.11). 
(FIGURE 2-1-2.)

- For $\sigma \in \Sigma_{\varphi}, \widetilde{\varphi}^{-1}\left(O_{\sigma}\right)=\widetilde{O_{\sigma}} \times F_{\sigma}^{c}$, where $\widetilde{O_{\sigma}}$ is a connected covering space of $O_{\sigma}$ of order Ind $(\sigma)$. Thus, the stratification of $X_{\Sigma}$ by $X_{\Sigma}-\widetilde{\varphi}\left(X_{\Sigma^{\prime}}\right)$ and the toric orbits $O_{\sigma}, \sigma \in \Sigma_{\varphi}$, of $X_{\Sigma_{\varphi}}$ in $\widetilde{\varphi}\left(X_{\Sigma^{\prime}}\right)$ gives a flattening stratification of $\tilde{\varphi}$.

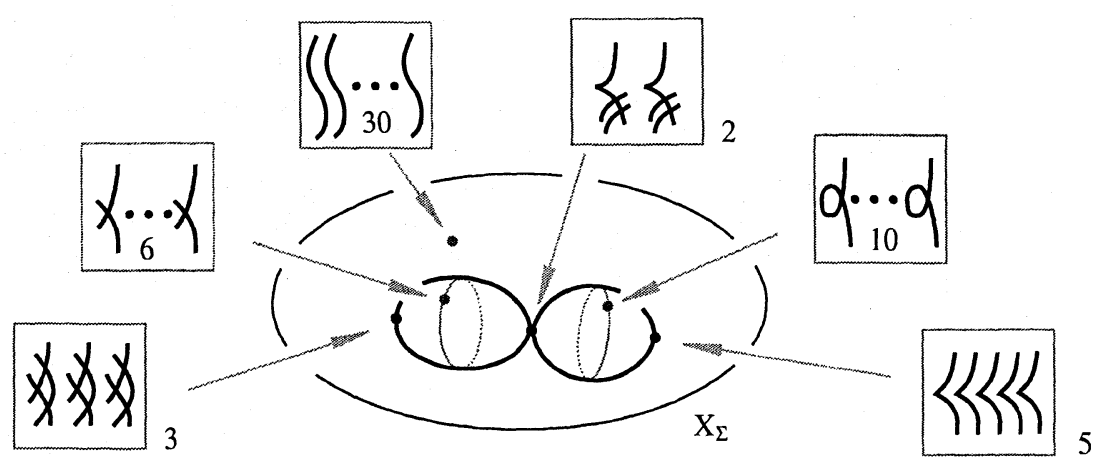

Figure 2: Figure 2-1-2. How the various fibers and their index of a toric morphism may look like is indicated. Though they could be wild in general, all the information is coded in the map between fans.

Remark 2.1.5. Here the term reducible toric variety means a reducible variety obtained by gluing a collection of toric varieties along some isomorphic toric orbits.

Proof of Proposition 2.1.4. Let $N_{\varphi}=N \cap \varphi\left(N_{\mathbb{R}}^{\prime}\right)$. Then the toric morphism $\tilde{\varphi}: X_{\Sigma^{\prime}} \rightarrow X_{\Sigma}$ is the composition of toric morphisms $X_{\Sigma^{\prime}} \stackrel{\widetilde{\varphi}}{\rightarrow} X_{\Sigma_{\varphi}} \stackrel{\tau}{\rightarrow} X_{\Sigma}$ induced from the maps of fans $\Sigma^{\prime} \stackrel{\varphi}{\rightarrow} \Sigma_{\varphi} \stackrel{\iota}{\rightarrow} \Sigma$ from linear maps $N^{\prime} \stackrel{\varphi}{\rightarrow} N_{\varphi} \stackrel{\iota}{\rightarrow} N$. Note that $\varphi\left(N^{\prime}\right)$ is a subgroup of $N_{\varphi}$ of finite index; hence, $\tilde{\varphi}: X_{\Sigma^{\prime}} \rightarrow X_{\Sigma_{\varphi}}$ is surjective. On the other hand, since $\Sigma_{\varphi}=\Sigma \cap N_{\varphi \mathbb{R}}, \widetilde{\iota}\left(X_{\Sigma_{\varphi}}\right)$ is the closure of the $T_{N_{\varphi}}$-orbit $T_{N_{\varphi}} \cdot x_{\{0\}}$ in $X_{\Sigma}$. Since it contains $T_{N_{\varphi}}$ as an $T_{N_{\varphi}}$-equivariant open dense subset, from the relation between torus actions and fans (e.g. [Fu]) one concludes that the closure is simply $X_{\Sigma_{\varphi}}$. This shows that $\tau: X_{\Sigma_{\varphi}} \rightarrow X_{\Sigma}$ is an inclusion. Together, one concludes that $\widetilde{\varphi}\left(X_{\Sigma^{\prime}}\right)$ is a subvariety of $X_{\Sigma}$ and it is realized as $X_{\Sigma_{\varphi}}$.

For the remaining part of the proof, we shall regard $\widetilde{\varphi}$ as a toric morphism $X_{\Sigma^{\prime}} \rightarrow X_{\Sigma_{\varphi}}$ induced by the map of fans $\varphi: \Sigma^{\prime} \rightarrow \Sigma_{\varphi}$. After relabelling to simplify the notation, without loss of generality we shall assume in the following discussion that $\varphi: N_{\mathbb{R}}^{\prime} \rightarrow N_{\mathbb{R}}$ is surjective and, hence $\Sigma_{\varphi}=\Sigma$. Note that in this case $\varphi\left(N^{\prime}\right)$ is a subgroup of finite index in $N$ and the induced group homomorphism $T_{N^{\prime}} \rightarrow T_{N}$ is surjective. 
Since $\widetilde{\varphi}$ is toric equivariant, it sends a toric orbit $O_{\sigma^{\prime}}$ in $X_{\Sigma^{\prime}}$ into a toric orbit $O_{\sigma}$ in $X_{\Sigma}$. The following lemma characterizes such pairs $\left(\sigma^{\prime}, \sigma\right)$.

Lemma 2.1.6 [orbit pair]. $\widetilde{\varphi}$ maps $O_{\sigma^{\prime}}$ to $O_{\sigma}$ if and only if $\varphi$ maps the interior of $\sigma^{\prime}$ to the interior of $\sigma$. When this happens, $\widetilde{\varphi}$ maps $O_{\sigma^{\prime}}$ surjectively to $O_{\sigma}$.

Proof. The if-part follows from the natural presentaion of orbits as affine subvarieties in a toric variety $([\mathrm{Fu}]): O_{\sigma^{\prime}}=\operatorname{Spec}\left(\mathbb{C}\left[{\sigma^{\prime}}^{\perp} \cap M^{\prime}\right]\right)$ and $O_{\sigma}=\operatorname{Spec}\left(\mathbb{C}\left[\sigma^{\perp} \cap M\right]\right)$. Note that, for each $\sigma^{\prime} \in \Sigma^{\prime}$, there is a unique $\sigma \in \Sigma$ such that the interior of $\sigma^{\prime}$ is mapped to the interior of $\sigma$. Thus, $\widetilde{\varphi}$ maps $O_{\sigma^{\prime}}$ to a particular $O_{\sigma}$ determined by $\varphi$. Since $X_{\Sigma}$ is stratified by toric orbits and the image of an orbit in $X_{\Sigma^{\prime}}$ can be contained only in a unique orbit in $X_{\Sigma}$, the only-if-part also follows. For such $\left(\sigma^{\prime}, \sigma\right)$, the surjectivity of $\tilde{\varphi}: O_{\sigma^{\prime}} \rightarrow O_{\sigma}$ follows from the surjectivity of $T_{N^{\prime}} \rightarrow T_{N}$. This concludes the proof.

To understand the fiber over each orbit, consider a homomorphism between lattices $\psi: N_{1} \rightarrow N_{2}$ with $\psi\left(N_{1}\right)$ of finite index in $N_{2}$. Let $\left[N_{2}: \psi\left(N_{1}\right)\right]$ be the index of $\psi\left(N_{1}\right)$ in $N_{2}$ and let $\widetilde{\psi}: T_{N_{1}} \rightarrow T_{N_{2}}$ be the induces morphism, where $T_{N_{1}}$, $T_{N_{2}}$ are regarded as the toric variety associated to the fans that consist only of the origin. One has then the following exact sequences

$$
\begin{aligned}
& 0 \longrightarrow \psi^{-1}(0) \longrightarrow N_{1} \longrightarrow \psi\left(N_{1}\right) \longrightarrow 0 \\
& 0 \longrightarrow \psi\left(N_{1}\right) \longrightarrow N_{2} \longrightarrow N_{2} / \psi\left(N_{1}\right) \rightarrow 0 \text {. }
\end{aligned}
$$

Note that $\psi^{-1}(0)$ is a lattice of rank $\operatorname{rank} N_{1}-\operatorname{rank} N_{2}$ and $N_{2} / \psi\left(N_{1}\right)$ is a finite abelian group. From the first exact sequence, the homomorphism $T_{N_{1}} \rightarrow T_{\psi\left(N_{1}\right)}$ is a bundle map with fiber $T_{\psi^{-1}(0)}$. From the second exact sequence, the homomorphism $T_{\psi\left(N_{1}\right)} \rightarrow T_{N_{2}}$ is a finite covering map with fiber a finite set of $\left[N_{2}: \psi\left(N_{1}\right)\right]$ many elements. Since the composition of these two morphisms gives the morphism $\widetilde{\psi}: T_{N_{1}} \rightarrow T_{N_{2}}$, one concludes that the fiber of $\widetilde{\psi}$ is a disjoint union of $\left[N_{2}: \psi\left(N_{1}\right)\right]$ copies of $T_{\psi^{-1}(0)}$. Notice that the same conclusion can also be phrased in terms of lattices $M_{1}, M_{2}$ dual to $N_{1}, N_{2}$ respectively.

Apply this to our problem. Let $\Sigma_{\sigma}^{\prime}$ be the set of cones in $\Sigma^{\prime}$ whose interior is mapped to the interior of $\sigma \in \Sigma$. Let $\sigma^{\prime} \in \Sigma_{\sigma}^{\prime}$. Since $\widetilde{\varphi}: O_{\sigma^{\prime}} \rightarrow O_{\sigma}$ is surjective, the image of the homomorphism behind, $\bar{\varphi}_{\sigma}^{\sigma^{\prime}}: N^{\prime} / N_{\sigma^{\prime}}^{\prime} \rightarrow N / N_{\sigma}$, must be of finite index in $N / N_{\sigma}$. (Equivalently, the induced homomorphism $\varphi_{\sigma^{\prime}}^{\dagger}: \sigma^{\perp} \cap M \rightarrow \sigma^{\perp} \cap M^{\prime}$ must be injective.) Since

$$
\bar{\varphi}_{\sigma}^{\sigma^{\prime-1}}(0)=\varphi^{-1}\left(N_{\sigma}\right) / N_{\sigma^{\prime}}^{\prime}
$$

the fiber $F_{\sigma}^{\sigma^{\prime}}$ of $\tilde{\varphi}$ over a point in $O_{\sigma}$ is thus a disjoint union of $\left[N / N_{\sigma}: \bar{\varphi}_{\sigma}^{\sigma^{\prime}}\left(N^{\prime} / N_{\sigma^{\prime}}^{\prime}\right)\right]$ copies of $T_{\varphi^{-1}\left(N_{\sigma}\right) / N_{\sigma^{\prime}}^{\prime}}$. 
The following two lemmas play an essential role in understanding fibers of $\tilde{\varphi}$ and their relations.

Definition/Lemma 2.1.7 [index of $\tilde{\varphi}$ over $O_{\sigma}$ ]. The image $\bar{\varphi}_{\sigma}^{\sigma^{\prime}}\left(N^{\prime} / N_{\sigma^{\prime}}^{\prime}\right)$ in $N / N_{\sigma}$ is independent of the choice of $\sigma^{\prime}$ in $\Sigma_{\sigma}^{\prime}$. Thus, the index $\left[N / N_{\sigma}: \bar{\varphi}_{\sigma}^{\sigma^{\prime}}\left(N^{\prime} / N_{\sigma^{\prime}}^{\prime}\right)\right]$ depends only on $\sigma$ and will be denoted by $\operatorname{Ind}(\sigma)$ and called the index of $\tilde{\varphi}$ over $O_{\sigma}$.

Proof. Let $\sigma_{1}^{\prime}, \sigma_{2}^{\prime} \in \Sigma_{\sigma}^{\prime}$. Assume first that $\sigma_{1}^{\prime}$ is in the face of $\sigma_{2}^{\prime}$. Then $N_{\sigma_{1}^{\prime}}^{\prime}$ is a subspace of $N_{\sigma_{2}^{\prime}}^{\prime}$ and one has the exact sequence:

$$
0 \longrightarrow N_{\sigma_{2}^{\prime}}^{\prime} / N_{\sigma_{1}^{\prime}}^{\prime} \longrightarrow N^{\prime} / N_{\sigma_{1}^{\prime}}^{\prime} \longrightarrow N^{\prime} / N_{\sigma_{2}^{\prime}}^{\prime} \longrightarrow 0
$$

Note that since $N_{\sigma_{2}^{\prime}}^{\prime}$ is mapped by $\bar{\varphi}_{\sigma}^{\sigma_{2}^{\prime}}$ to the 0 in $N / N_{\sigma}, N_{\sigma_{2}^{\prime}}^{\prime} / N_{\sigma_{1}^{\prime}}^{\prime}$ as a subspace in $N^{\prime} / N_{\sigma_{1}^{\prime}}^{\prime}$ is mapped by $\bar{\varphi}_{\sigma}^{\sigma_{1}^{\prime}}$ also to the 0 in $N / N_{\sigma}$. Since $N^{\prime} / N_{\sigma_{2}^{\prime}}^{\prime}=$ $\left(N^{\prime} / N_{\sigma_{1}^{\prime}}^{\prime}\right) /\left(N_{\sigma_{2}^{\prime}}^{\prime} / N_{\sigma_{1}^{\prime}}^{\prime}\right)$ canonically, $\bar{\varphi}_{\sigma}^{\sigma_{1}^{\prime}}$ descends to $\bar{\varphi}_{\sigma}^{\sigma_{2}^{\prime}}$ also canonically after taking the quotient by $N_{\sigma_{2}^{\prime}}^{\prime} / N_{\sigma_{1}^{\prime}}^{\prime}$. This shows that $\bar{\varphi}_{\sigma}^{\sigma_{1}^{\prime}}\left(N^{\prime} / N_{\sigma_{1}^{\prime}}^{\prime}\right)=\bar{\varphi}_{\sigma_{2}^{\prime}}^{\sigma_{2}^{\prime}}\left(N^{\prime} / N_{\sigma_{2}^{\prime}}^{\prime}\right)$ if $\sigma_{1}^{\prime}$ is in the face of $\sigma_{2}^{\prime}$.

For general $\sigma_{1}^{\prime}, \sigma_{2}^{\prime} \in \Sigma_{\sigma}^{\prime}$, let $\stackrel{\circ}{\sigma}$ be the interior of $\sigma$ and regard $\varphi$ as a map $N_{\mathbb{R}}^{\prime} \rightarrow N_{\mathbb{R}}$. Then, since $\varphi^{-1}(\stackrel{\circ}{\sigma})$ is connected, one can find a finite sequence of cones $\sigma_{1}^{\prime}, \tau_{1}^{\prime}, \cdots, \tau_{s}^{\prime}, \sigma_{2}^{\prime}$ in $\Sigma_{\sigma}^{\prime}$ such that each element in the sequence either contains as a face or is in the face of the next element. By transitivity, this shows that $\bar{\varphi}_{\sigma}^{\sigma_{1}^{\prime}}\left(N^{\prime} / N_{\sigma_{1}^{\prime}}^{\prime}\right)=\bar{\varphi}_{\sigma}^{\sigma_{2}^{\prime}}\left(N^{\prime} / N_{\sigma_{2}^{\prime}}^{\prime}\right)$ and we conclude the proof.

Lemma 2.1.8. If $\tau$ is in the face of $\sigma$, then $\operatorname{Ind}(\tau)$ is an integral multiple of $\operatorname{Ind}(\sigma)$.

Proof. Since $\tau$ is in the face of $\sigma, \varphi^{-1}(\tau)$ must lie in the boundary of $\varphi^{-1}(\sigma)$. Since both $\varphi^{-1}(\tau)$ and $\varphi^{-1}(\sigma)$ are unions of cones, each cone in $\varphi^{-1}(\tau)$, in particular each $\tau^{\prime} \in \Sigma_{\tau}^{\prime}$, must lie in the face of some cone $\sigma^{\prime}$ in $\Sigma_{\sigma}^{\prime}$. Consider now the following diagram of abelian groups with exact rows:

$$
\begin{aligned}
& 0 \rightarrow N_{\sigma^{\prime}}^{\prime} / N_{\tau^{\prime}}^{\prime} \longrightarrow N^{\prime} / N_{\tau^{\prime}}^{\prime} \longrightarrow N^{\prime} / N_{\sigma^{\prime}}^{\prime} \longrightarrow 0 \\
& \alpha \downarrow \quad \bar{\varphi}_{\tau}^{\tau^{\prime}} \downarrow \quad \bar{\varphi}_{\sigma}^{\sigma^{\prime}} \downarrow \\
& 0 \rightarrow N_{\sigma} / N_{\tau} \longrightarrow N / N_{\tau} \longrightarrow N / N_{\sigma} \longrightarrow 0 .
\end{aligned}
$$

Note that the diagram commutes since all the homomorphisms involved are induced from the morphism $\varphi: N^{\prime} \rightarrow N$. By the Snake Lemma in homological algebra ([Ei]), one has the exact sequence

$$
0 \rightarrow \operatorname{ker} \alpha \rightarrow \operatorname{ker} \bar{\varphi}_{\sigma}^{\sigma^{\prime}} \rightarrow \operatorname{ker} \bar{\varphi}_{\sigma}^{\sigma^{\prime}} \rightarrow \operatorname{coker} \alpha \rightarrow \operatorname{coker} \bar{\varphi}_{\tau}^{\tau^{\prime}} \rightarrow \operatorname{coker} \bar{\varphi}_{\sigma}^{\sigma^{\prime}} \rightarrow 0
$$


which implies that $\operatorname{Ind}(\sigma)$ divides $\operatorname{Ind}(\tau)$ by considering the cardinality of the last three terms. This concludes the proof.

We are now ready to understand how the toroidal pieces from the orbit-to-orbit fibrations are joined together to form a complete fiber.

Let $\sigma \in \Sigma$. By assumption, $\Sigma_{\sigma}^{\prime}$ is non-empty. We shall regard $\Sigma_{\sigma}^{\prime}$ as a subfan in $\Sigma^{\prime}$. Recall the construction of the star of a cone in toric geometry. Here we need a relative version defined as follows.

Let $\tau^{\prime} \in \Sigma_{\sigma}^{\prime}$ and $\left\{\sigma_{1}^{\prime}, \sigma_{2}^{\prime}, \cdots\right\}$ be the set of cones in $\Sigma_{\sigma}^{\prime}$ that contains $\tau^{\prime}$ as a face. Then each $\sigma_{i}^{\prime}$ determines a cone $\overline{\sigma_{i}^{\prime}}$ in $\varphi^{-1}\left(\left(N_{\sigma}\right)_{\mathbb{R}}\right) /\left(N_{\tau^{\prime}}^{\prime}\right)_{\mathbb{R}}$, defined by

$$
\overline{\sigma_{i}^{\prime}}=\left(\sigma_{i}^{\prime}+\left(N_{\tau^{\prime}}^{\prime}\right)_{\mathbb{R}}\right) /\left(N_{\tau^{\prime}}^{\prime}\right)_{\mathbb{R}} \cdot
$$

Note that $\sigma_{i}^{\prime}+\left(N_{\tau^{\prime}}^{\prime}\right)_{\mathbb{R}}$ is contained in $\varphi^{-1}\left(\left(N_{\sigma}\right)_{\mathbb{R}}\right)$ since $\tau^{\prime}, \sigma_{i}^{\prime} \in \Sigma_{\sigma}^{\prime}$. Thus, $\left\{\overline{\sigma_{1}^{\prime}}, \overline{\sigma_{2}^{\prime}}, \cdots\right\}$ defines a fan in $\varphi^{-1}\left(\left(N_{\sigma}\right)_{\mathbb{R}}\right) /\left(N_{\tau^{\prime}}^{\prime}\right)_{\mathbb{R}}$.

Definition 2.1.9 [relative star]. The fan in $\varphi^{-1}\left(\left(N_{\sigma}\right)_{\mathbb{R}}\right) /\left(N_{\tau^{\prime}}^{\prime}\right)_{\mathbb{R}}$ constructed above will be called the relative star of $\tau^{\prime}$ over $\sigma$ and will be denoted by $\operatorname{Star}_{\sigma}\left(\tau^{\prime}\right)$.

Definition 2.1.10 [primitive cone]. A cone $\tau^{\prime} \in \Sigma_{\sigma}^{\prime}$ is called primitive with respect to $\varphi$ if none of the faces of $\tau^{\prime}$ are in $\Sigma_{\sigma}^{\prime}$. The set of primitive cones in $\Sigma_{\sigma}^{\prime}$ will be denoted by $\Sigma_{\sigma}^{\prime \circ}$.

(Cf. Figure 2-1-3.)

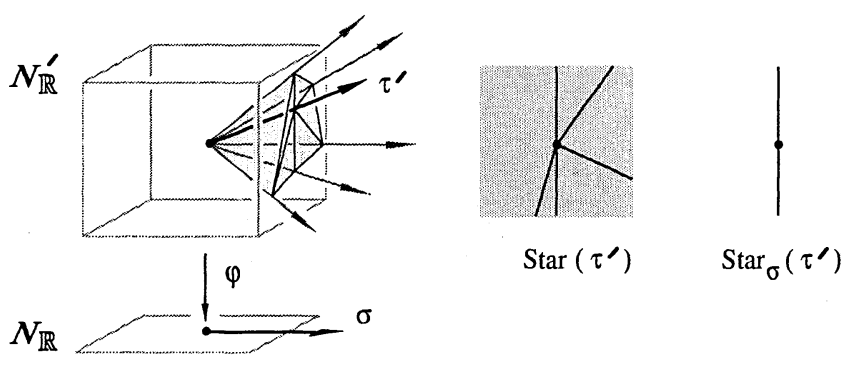

Figure 3: FIgURE 2-1-3. A comparison of the relative star $\operatorname{Star}_{\sigma}\left(\tau^{\prime}\right)$ with respect to a map of fans $\varphi$ and the usual star $\operatorname{Star}\left(\tau^{\prime}\right)$.

The following lemma justifies the two definitions above.

Lemma 2.1.11 [connected/irreducible components of fiber]. Given $y \in O_{\sigma}$, let $F_{\sigma}^{c}$ be a connected component of $\widetilde{\varphi}^{-1}(y)$. Then 
(a) There is a 1-1 correspondence between the irreducible components of the fiber $F_{\sigma}^{c}$ over $O_{\sigma}$ and the elements in $\Sigma_{\sigma}^{\prime \circ}$.

(b) Let $\tau^{\prime} \in \Sigma_{\sigma}^{\prime \circ}$, then the component $F_{\sigma}^{\tau^{\prime}}$ of $F_{\sigma}^{c}$ associated to $\tau^{\prime}$ is the toric variety associated to the fan $\operatorname{Star}_{\sigma}\left(\tau^{\prime}\right)$ in $\varphi^{-1}\left(\left(N_{\sigma}\right)_{\mathbb{R}}\right) /\left(N_{\tau^{\prime}}^{\prime}\right)_{\mathbb{R}}$. It has (complex) dimension: $\operatorname{codim} \tau^{\prime}-\operatorname{codim} \sigma$.

(c) If $\tau_{1}^{\prime}, \cdots, \tau_{s}^{\prime} \in \Sigma_{\sigma}^{\prime \circ}$ generate a cone $\sigma^{\prime}$ in $\Sigma^{\prime}$, then $\sigma^{\prime} \in \Sigma_{\sigma}^{\prime}$ and $\bigcap_{i=1}^{s} F_{\sigma}^{\tau_{i}^{\prime}}=\operatorname{Star}_{\sigma}\left(\sigma^{\prime}\right)$.

(d) The whole fiber $F_{\sigma}$ over a point in $O_{\sigma}$ is the disjoint union of Ind $(\sigma)$ many copies of $F_{\sigma}^{c}$.

Proof. (a) The $1-1$ correspondence: By definition, any non-primitive element $\sigma^{\prime}$ in $\Sigma_{\sigma}^{\prime}$ must contain some primitive element $\tau^{\prime}$ in its face. This implies that $F_{\sigma}^{\sigma^{\prime}}$ is in the closure of $F_{\sigma}^{\tau^{\prime}}$. On the other hand, for a primitive $\tau^{\prime}$ in $\Sigma_{\sigma}^{\prime}$ the fiber $F_{\sigma}^{\tau^{\prime}}$ cannot be contained in the closure of $F_{\sigma}^{\sigma^{\prime}}$ for any $\sigma^{\prime} \neq \tau^{\prime}$. This shows that $F_{\sigma}^{\tau^{\prime}}$ is a maximal stratum in $F_{\sigma}^{c}$ and, hence, its closure must be a component of $F_{\sigma}^{c}$. Together, these prove the $1-1$ corrrespondence.

(b) The toric geometry of a component: Let $\tau^{\prime}$ be a primitive element in $\Sigma_{\sigma}^{\prime}$. Then the closure $\overline{F_{\sigma}^{\tau^{\prime}}}$ consists of $F_{\sigma}^{\tau^{\prime}}$ and all $F_{\sigma}^{\sigma^{\prime}}$ with $\sigma^{\prime}$ containing $\tau^{\prime}$ in its face. By construction, this is exactly the fan $\operatorname{Star}_{\sigma}\left(\tau^{\prime}\right)$ in $\varphi^{-1}\left(\left(N_{\sigma}\right)_{\mathbb{R}}\right) /\left(N_{\tau^{\prime}}^{\prime}\right)_{\mathbb{R}}$, which has dimension $\operatorname{codim} \tau^{\prime}-\operatorname{codim} \sigma$. This proves Part (b).

(c) The intersection of irreducible components: This follows from the definition of relative stars and the order-reversing correspondence between cones $\sigma^{\prime}$ in $\Sigma_{\sigma}^{\prime}$ and toric varieties associated to $\operatorname{Star}_{\sigma}\left(\sigma^{\prime}\right)$.

(d) The number of connected components: Note that the fiber $F_{\sigma}$ over a point in $O_{\sigma}$ is naturally stratified by complex tori of the form $\left(\mathbb{C}^{*}\right)^{r}$. Lemma 2.1.6 implies that each $\sigma^{\prime}$ contributes to $F_{\sigma}$ exactly $\operatorname{Ind}(\sigma)$-many strata $\left(\mathbb{C}^{*}\right)^{r}$, where $r=\operatorname{codim} \sigma^{\prime}-$ $\operatorname{codim} \sigma_{\sigma}$. Each connected components of $F_{\sigma}$ will contain at least one of them. On the other hand, the restriction of the map $\tilde{\varphi}: \widetilde{\varphi}^{-1}\left(O_{\sigma}\right) \rightarrow O_{\sigma}$ factors through a covering morphism $\left(\mathbb{C}^{*}\right) \rightarrow O_{\sigma}$ of degree $\operatorname{Ind}(\sigma)$. Together this shows that $F_{\sigma}$ must have exactly Ind $(\sigma)$-many connected components.

This concludes the proof.

This justifies the description of fibers of a toric morphism in Proposition 2.1.4.

Finally, let us consider the induced stratification of $\widetilde{\varphi}$ by toric orbits $O_{\sigma}$ of $X_{\Sigma}$. Observe first the following commutative diagram of maps of fans

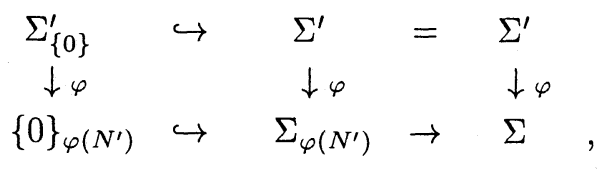


where $\{0\}_{\varphi\left(N^{\prime}\right)}$ is the fan in $\varphi\left(N^{\prime}\right)_{\mathbb{R}}$ that consists only of $\{0\}, \Sigma_{\{0\}}^{\prime}$ is regarded as a fan in $N_{\mathbb{R}}^{\prime}$, and $\Sigma_{\varphi\left(N^{\prime}\right)}$ is the naturally induced fan in $\varphi\left(N^{\prime}\right)_{\mathbb{R}}$ from $\Sigma$ in $N_{\mathbb{R}}$. By taking the toric variety associated to the fans in the diagram, one obtains the torically equivariant commutative diagram

$\begin{array}{cccc}\widetilde{O_{\{0\}}} \times F_{\{0\}}^{c} & \hookrightarrow & X_{\Sigma^{\prime}} \\ \downarrow \widetilde{\varphi} & & \downarrow \widetilde{\varphi} \\ O_{\{0\}} & \hookrightarrow & X_{\Sigma},\end{array}$

where $O_{\{0\}}$ is the open dence $T_{N}$-orbit in $X_{\Sigma}$ and $\widetilde{O_{\{0\}}} \stackrel{\widetilde{\varphi}}{\rightarrow} O_{\{0\}}$ is a covering map of order $\left[N: \varphi\left(N^{\prime}\right)\right]=\operatorname{Ind}(\{0\})$ This shows that $\tilde{\varphi}^{-1}\left(O_{\{0\}}\right)=\widetilde{O_{\{0\}}} \times F_{\{0\}}^{c}$, as claimed. For the stratum over general orbit $O_{\sigma}, \sigma \in \Sigma$, let $\tau^{\prime}$ be a primitive element in $\Sigma_{\sigma}^{\prime}$. Then the discussion above applied to the following commutative diagram of maps of fans

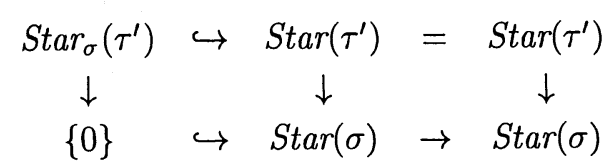

from the maps of lattices induced by $\varphi$ (with the fans in the above diagram lying in the realization of the lattices in the diagram below at the same relative positions)

$$
\begin{array}{ccccc}
N^{\prime} / N_{\tau^{\prime}}^{\prime} & = & N^{\prime} / N_{\tau^{\prime}}^{\prime} & = & N^{\prime} / N_{\tau^{\prime}}^{\prime} \\
\downarrow \bar{\varphi}_{\sigma}^{\tau^{\prime}} & & \downarrow \bar{\varphi}_{\sigma}^{\tau^{\prime}} & & \downarrow \bar{\varphi}_{\sigma}^{\tau^{\prime}} \\
\bar{\varphi}_{\sigma}^{\tau^{\prime}}\left(N^{\prime} / N_{\tau^{\prime}}^{\prime}\right) & = & \bar{\varphi}_{\sigma}^{\tau^{\prime}}\left(N^{\prime} / N_{\tau^{\prime}}^{\prime}\right) & \hookrightarrow & N / N_{\sigma}
\end{array}
$$

shows that $\widetilde{O_{\sigma}} \times F_{\sigma}^{\tau^{\prime}}$ is contained in $\widetilde{\varphi}^{-1}\left(O_{\sigma}\right)$, where $\widetilde{O_{\sigma}} \rightarrow O_{\sigma}$ is a covering map of order $\operatorname{Ind}(\sigma)$. From the pasting of the irreducible components $F_{\sigma}^{\tau^{\prime}}$ to form $F_{\sigma}^{c}$ discussed earlier, one concludes that $\widetilde{\varphi}^{-1}\left(O_{\sigma}\right)=\widetilde{O_{\sigma}} \times F_{\sigma}^{c}$.

This concludes the proof of Proposition 2.1.4.

Remark 2.1.12. If $\left[N: \varphi\left(N^{\prime}\right)\right]<\infty$, then

$$
\operatorname{Ind}(\{0\})=\left[N: \varphi\left(N^{\prime}\right)\right]=\operatorname{Ind}(\sigma) \cdot\left[N_{\sigma}: N_{\sigma} \cap \varphi\left(N^{\prime}\right)\right] .
$$

In particular, if $\varphi$ is surjective, then $\operatorname{Ind}(\sigma)=1$ for all $\sigma \in \Sigma$.

\section{Characterization of toric fibrations.}

To characterize a fibration, let us assume that $\tilde{\varphi}: X_{\Sigma^{\prime}} \rightarrow X_{\Sigma}$ is surjective. Let $F_{\sigma}^{\tau^{\prime}}$ be an irreducible component of the connected fiber $F_{\sigma}^{c}$ over $O_{\sigma} \subset X_{\Sigma}$. Then, from Lemma 2.1.11,

$$
\begin{gathered}
\operatorname{dim} F_{\sigma}^{\tau^{\prime}}=\operatorname{dim}\left(\varphi^{-1}\left(\left(N_{\sigma}\right)_{\mathbb{R}}\right) /\left(N_{\tau^{\prime}}^{\prime}\right)_{\mathbb{R}}\right)=\operatorname{dim} \varphi^{-1}(0)+\operatorname{dim}\left(N_{\sigma}\right)_{\mathbb{R}}-\operatorname{dim}\left(N_{\tau^{\prime}}^{\prime}\right)_{\mathbb{R}} \\
\geq \operatorname{dim} \varphi^{-1}(0)=\operatorname{dim} F_{0}=\operatorname{dim} X_{\Sigma^{\prime}}-\operatorname{dim} X_{\Sigma}
\end{gathered}
$$


where $F_{0}^{c}$ is a connected component of a generic fiber and we use the fact that, for $\tau^{\prime}$ primitive, the map $\varphi_{\mathbb{R}}:\left(N_{\tau^{\prime}}^{\prime}\right)_{\mathbb{R}} \rightarrow\left(N_{\sigma}\right)_{\mathbb{R}}$ is injective. From this, one concludes :

Corollary 2.1.13 [fibration]. Let $\tilde{\varphi}: X_{\Sigma^{\prime}} \rightarrow X_{\Sigma}$ is a surjective toric morphism. Then $\tilde{\varphi}$ is a fibration, in the sense that all the irreducible components of fibers of $\tilde{\varphi}$ have the same dimension, $\operatorname{dim} X_{\Sigma^{\prime}}-\operatorname{dim} X_{\Sigma}$, if and only if the map of cones $\varphi_{\mathbb{R}}: \tau^{\prime} \rightarrow \sigma$ is a bijection for every $\tau^{\prime} \in \Sigma_{\sigma}^{\prime \circ}$.

In particular, since a ray in $\Sigma^{\prime}(1)-\varphi^{-1}(0)$ must be a primitive cone, if $\widetilde{\varphi}$ is a fibration, then $\varphi$ maps $\Sigma^{\prime}(1)$ to $\Sigma(1)$ surjectively.

\section{Dual picture via polytopes in $M$ lattice.}

As the above proposition indicates, the study of toric morphisms via fans in the $N$ lattice is very natural. Nevertheless, for projective toric varieties, it is convenient to have the dual polyhedral picture in the $M$ lattice in mind. We summarize some essential features here. They follow directly from the proof of Proposition 2.1.4 by taking dual and tracing definitions.

Let $\tilde{\varphi}: X_{\Sigma^{\prime}} \rightarrow X_{\Sigma}$ be a toric morphism between projective toric varieties. Without loss of generality, we may assume that $\varphi_{\mathbb{R}}: N_{\mathbb{R}}^{\prime} \rightarrow N_{\mathbb{R}}$ is surjective. Note that this implies that $\varphi^{\dagger}: M \rightarrow M^{\prime}$ is injective. Let $\Delta^{\prime}$ (resp. $\Delta$ ) be a convex polytope in $M_{\mathbb{R}}^{\prime}$ whose normal fan is $\Sigma^{\prime}$ (resp. $\Sigma$ ). The face of $\Delta$ whose normal cone is $\sigma$ will be denoted by $\Theta_{\sigma}$. Note that, by definition, the face associated to $\{0\}$ in $N_{\mathbb{R}}$ is $\Delta$. Similarly for the face $\Theta_{\sigma^{\prime}}$ of $\Delta^{\prime}$.

The following equivalences play a key role in translating the fan picture to the dual polytope picture:

$$
\begin{aligned}
& \varphi\left(\text { interior of } \sigma^{\prime}\right) \subset \text { interior of } \sigma . \\
\Longleftrightarrow & \left\langle u, \varphi\left(v^{\prime}\right)\right\rangle>0 \text { for all } u \in \sigma^{\vee}-\sigma^{\perp} \text { and } v^{\prime} \text { in the interior of } \sigma^{\prime} . \\
\Longleftrightarrow & \left\langle\varphi^{\dagger}(u), v^{\prime}\right\rangle>0 \text { for all } u \in \sigma^{\vee}-\sigma^{\perp} \text { and } v^{\prime} \text { in the interior of } \sigma^{\prime} . \\
\Longleftrightarrow & \left.\varphi^{\dagger}\left(\sigma^{\vee}-\sigma^{\perp}\right) \subset \sigma^{\vee}-\sigma^{\perp} . \quad \text { (Note that } \varphi^{\dagger}\left(\sigma^{\perp}\right) \subset \sigma^{\perp} .\right)
\end{aligned}
$$

For $\sigma \in \Sigma$, define the $\sigma$-lighted part $\Delta_{\sigma}^{\prime} \subset \Delta^{\prime}$ associated to a face $\Theta_{\sigma} \subset \Delta$ by

$$
\Delta_{\sigma}^{\prime}=\left\{\Theta_{\sigma^{\prime}} \in \Delta^{\prime} \mid \varphi^{\dagger}\left(\sigma^{\vee}-\sigma^{\perp}\right) \subset \sigma^{\prime \vee}-\sigma^{\perp}\right\} .
$$

(The terminology is inspired by FiguRE 2-1-4.) Note that, for an interior point $p \in \Theta_{\sigma^{\prime}}$, the unridged tangent cone $T_{p} \Delta^{\prime}-T_{p} \Theta_{\sigma^{\prime}}$ at $p$ is isomorphic to $\sigma^{\prime \vee}-\sigma^{\prime \perp}$. Thus, $\Delta_{\sigma}^{\prime}$ is the polyhedral sub-complex of $\Delta^{\prime}$ that consists of faces, the unridged tangent cone at an interior point of which contains $\varphi^{\dagger}\left(\sigma^{\vee}-\sigma^{\perp}\right)$. By definition, $\Theta_{\sigma^{\prime}} \in \Delta_{\sigma}^{\prime}$ if and only if the interior of $\sigma^{\prime}$ is mapped by $\varphi$ to the interior of $\sigma$. If one regards $\Delta^{\prime}$ as a collection of combinatorial polytopes related by inclusion relations, then $\Delta^{\prime}$ is decomposed into a disjoint union $\sqcup_{\sigma \in \Sigma} \Delta_{\sigma}^{\prime}$. (FIGURE 2-1-4.) 


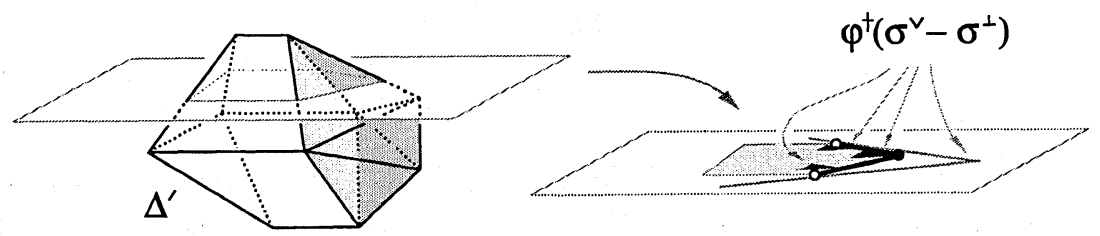

Figure 4: Figure 2-1-4. The $\sigma$-lighted part $\Delta_{\sigma}^{\prime} \subset \Delta^{\prime}$ associated to a face $\Theta_{\sigma} \subset \Delta$ is indicated by the shaded polyhedral subcomplex. A cross-section to indicate why these faces are $\sigma$-lighted is also indicated. (Cf. Forward light cones, domain of influence, ..., in general relativity.)

Lemma 2.1.14 [polytope for relative star]. Let $\Theta_{\tau^{\prime}} \in \Delta_{\sigma}^{\prime}$, regarded as a polytope in $\tau^{\perp}$. Let $\overline{\varphi_{\sigma}^{\dagger}} \tau^{\prime}: \tau^{\prime \perp} \rightarrow \tau^{\prime \perp} / \varphi^{\dagger}\left(\sigma^{\perp}\right)$ be the quotient map. Then the normal fan of the polytope $\overline{\Theta_{\tau^{\prime}}}=\overline{\varphi^{\dagger}}{ }_{\sigma}^{\tau^{\prime}}\left(\Theta_{\tau^{\prime}}\right)$ in $\tau^{{ }^{\perp}} / \varphi^{\dagger}\left(\sigma^{\perp}\right)$ is the relative star $\operatorname{Star}_{\sigma}\left(\tau^{\prime}\right)$. (FIGURE 2-1-5.)

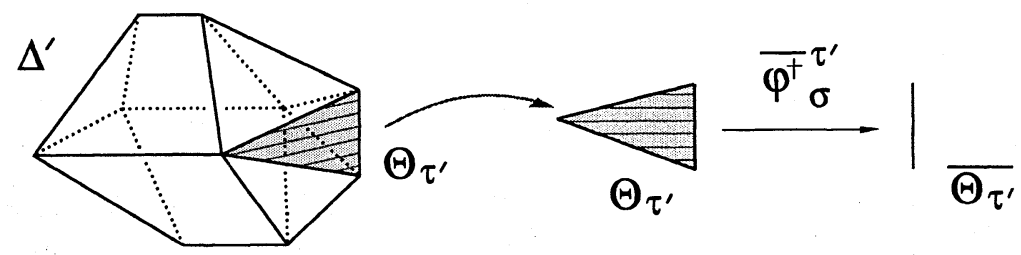

Figure 5: Figure 2-1-5. The polytope $\overline{\Theta_{\tau^{\prime}}}$ in $\tau^{\prime \perp} / \varphi^{\dagger}\left(\sigma^{\perp}\right)$ whose normal fan is the relative star $\operatorname{Star}_{\sigma}\left(\tau^{\prime}\right)$ is indicated. (In the picture, $\varphi^{\dagger}\left(\sigma^{\perp}\right)$ is realized as a foliation of $\tau^{\prime \perp}$.)

Proof. Recall Definition 2.1.9 of relative stars. Let $\tau^{\prime} \in \Sigma_{\sigma}^{\prime}$; then there is a nondegenerate pairing $\varphi^{-1}\left(N_{\sigma}\right) / N_{\tau^{\prime}}^{\prime} \times \tau^{\prime \perp} / \varphi^{\dagger}\left(\sigma^{\perp}\right) \longrightarrow \mathbb{Z}$ induced by the restriction of the canonical pairing $N^{\prime} \times M^{\prime} \longrightarrow \mathbb{Z}$ to $\varphi^{-1}\left(N_{\sigma}\right) \times \tau^{\prime \perp} \longrightarrow \mathbb{Z}$. Consequently, if $\sigma^{\prime} \in \Sigma_{\sigma}^{\prime}$ contains $\tau^{\prime}$ in its face, then the dual cone of $\overline{\sigma^{\prime}}=\left(\sigma^{\prime}+\left(N_{\tau^{\prime}}^{\prime}\right)_{\mathbb{R}}\right) /\left(N_{\tau^{\prime}}^{\prime}\right)_{\mathbb{R}} \subset$ $\varphi^{-1}\left(\left(N_{\sigma}\right)_{\mathbb{R}}\right) /\left(N_{\tau^{\prime}}^{\prime}\right)_{\mathbb{R}}$ is simply $\left(\left(\sigma^{\prime \vee} \cap{\tau^{\prime}}^{\perp}\right)+\varphi^{\dagger}\left(\sigma^{\perp}\right)\right) / \varphi^{\dagger}\left(\sigma^{\perp}\right) \subset \tau^{\perp} / \varphi^{\dagger}\left(\sigma^{\perp}\right)$, which is the tangent cone of $\overline{\Theta_{\tau^{\prime}}}$ at an interior point of the face $\overline{\varphi^{\dagger}}{ }_{\sigma}^{\prime}\left(\Theta_{\sigma^{\prime}}\right) \subset \overline{\Theta_{\tau^{\prime}}}$. This gives a correspondence from $\operatorname{Star}_{\sigma}\left(\tau^{\prime}\right)$ to the set of faces of $\overline{\Theta_{\tau^{\prime}}}$. This also implies that the normal fan of $\overline{\Theta_{\tau^{\prime}}}$ contains $\operatorname{Star}_{\sigma}\left(\tau^{\prime}\right)$. Since both fans are complete, they must be identical. This concludes the proof.

Similarly, one has the dual characterization for primitive elements $\sigma^{\prime}$ in $\Sigma_{\sigma}^{\prime}$ : 
Definition 2.1.15 [primitive face]. A face $\Theta_{\sigma^{\prime}}$ in $\Delta_{\sigma}^{\prime}$ is called primitive if it is not in the boundary of any other face in $\Delta_{\sigma}^{\prime}$.

One can check that $\sigma^{\prime}$ is a primitive elememt in $\Sigma_{\sigma}^{\prime}$ if and only if $\Theta_{\sigma^{\prime}}$ is a primitive face of $\Delta_{\sigma}^{\prime}$. The following corollary follows immediately from Lemma 2.1.11 and Lemma 2.1.14 (cf. the notations in Lemma 2.1.11).

Corollary 2.1.16 [polytope for irreducible component of fiber]. (a) There is a $1-1$ correspondence between the primitive faces in $\Delta_{\sigma}^{\prime}$ and the irreducible components of the fiber $F_{\sigma}^{c}$ of $\widetilde{\varphi}$ over a point in $O_{\sigma}$. (b) Let $\Theta_{\tau^{\prime}}$ be a primitive face of $\Delta_{\sigma}^{\prime}$. Then the corresponding irreducible component $F_{\sigma}^{\tau^{\prime}}$ in $F_{\sigma}^{c}$ is the toric variety associated to the normal fan of the polytope $\overline{\Theta_{\tau^{\prime}}}$ in $\tau^{\prime \perp} / \varphi^{\dagger}\left(\sigma^{\perp}\right)$.

In particular, for the generic fiber of the morphism $\widetilde{\varphi},\{0\}^{\vee}=M$ and $\Delta_{\{0\}}^{\prime}$ consists of $\Delta^{\prime}$ and all the faces $\Theta_{\sigma^{\prime}}$ of $\Delta^{\prime}$ with $\sigma^{\prime \perp} \supset \varphi^{\dagger}(M)$. The normal fan of the projection of $\Delta_{\{0\}}^{\prime}$ in $\left(M^{\prime} / \varphi^{\dagger}(M)\right)_{\mathbb{R}}$ is exactly $\Sigma_{\{0\}}^{\prime}$ as discussed earlier. (Cf. [A-K-M-S].)

\subsection{Examples of toric morphisms and fibers.}

In this subsection, we give two examples of toric morphisms and their fibers to illustrate the previous discussions. The second example is a toric fibration from [B-C-dlO-G] and will be the major example for later discussions of this article.

Example 2.2.1 $\left[\tilde{\varphi}: X_{\Sigma^{\prime}} \rightarrow \mathbb{C P}^{1}\right]$. Let $\Sigma=\left\{\sigma_{+},\{0\}, \sigma_{-}\right\}$be the fan for $\mathbb{C P}^{1}$. Then one has the corresponding decomposition $\Sigma^{\prime}=\Sigma_{\sigma_{+}}^{\prime} \cup \Sigma_{\{0\}}^{\prime} \cup \Sigma_{\sigma_{-}}^{\prime}$. Let $\Sigma^{\prime}(1)=$ $\Sigma_{+}^{\prime}(1) \cup \Sigma_{0}^{\prime}(1) \cup \Sigma_{-}(1)$ be the corresponding decomposition. Since $\sigma_{+}$and $\sigma_{-}$are 1-dimensional and cones in $\Sigma^{\prime}$ are strongly convex, the set of primitive elements in $\Sigma_{\sigma_{+}}^{\prime}\left(\right.$ resp. $\left.\Sigma_{\sigma_{-}}^{\prime}\right)$ is exactly $\Sigma_{+}^{\prime}(1)$ (resp. $\Sigma_{-}^{\prime}(1)$ ). Also, since $\sigma_{ \pm}$is a maximal cone, for $\sigma^{\prime} \in \Sigma_{ \pm}^{\prime}(1)$ the relative star $\operatorname{Star}_{\sigma_{ \pm}}\left(\sigma^{\prime}\right)$ coincides with the usual star $\operatorname{Star}\left(\sigma^{\prime}\right)$. Consequently, all the irreducible components of fibers of $\widetilde{\varphi}$ are of codimension 1 in $X_{\Sigma^{\prime}}$. Note that in this case $\mathbb{C P}^{1}$ has only three distinct orbits: $\mathbb{C}^{*}, x_{+}$, and $x_{-}$. Thus, there are at most three different kinds of fibers for $\widetilde{\varphi}$ :

(1) generic fiber: a connected component is given by the toric variety $\Sigma^{\prime} \cap$ $\varphi^{-1}(0)$; there are $\left[N: \varphi\left(N^{\prime}\right)\right]$-many connected components.

(2) the fiber over $x_{+}$: Since $N / N_{\sigma_{+}}=\{0\}$, by Lemma 2.1 .11 the fiber can have only one connected component. The set of irreducible components of the fiber is given then by $\left\{\operatorname{Star}\left(\sigma^{\prime}\right) \mid \sigma^{\prime} \in \Sigma_{\sigma_{+}}^{\prime}\right\}$.

(3) the fiber over $x_{-}$: similar to (2) with the label + replaced by - . 
For the dual picture, assume that $X_{\Sigma^{\prime}}$ is projective and $\Sigma^{\prime}$ is the normal fan of a convex polytope $\Delta^{\prime}$ in $M_{\mathbb{R}}^{\prime}$. Then $\Delta^{\prime}$ is decomposed into $\Delta_{\sigma_{+}}^{\prime}, \Delta_{\{0\}}^{\prime}$, and $\Delta_{\sigma_{-}}^{\prime}$. The set of primitive faces in $\Delta_{\sigma_{+}}^{\prime}$ is exactly the set of maximal faces of $\Delta_{\sigma_{+}}^{\prime}$. Similarly for $\Delta_{\sigma_{-}}^{\prime}$. The generic fiber of $\tilde{\varphi}$ corresponds to the projection of $\Delta^{\prime}$ in $\left(M^{\prime} / \varphi^{\dagger}(M)\right)_{\mathbb{R}}$ For the fiber $F_{x^{+}}$over $x_{+}$, since $\sigma_{+}^{\perp}=\{0\}$, each maximal face $\Theta_{\sigma^{\prime}}$ in $\Delta_{\sigma_{+}}^{\prime}$ as a polytope in $\sigma^{\prime \perp}$ corresponds to an irreducible component of $F_{x_{+}}$. Similarly for the fiber $F_{x_{-}}$over $x_{-}$. (Cf. Figure 2-2-1.)
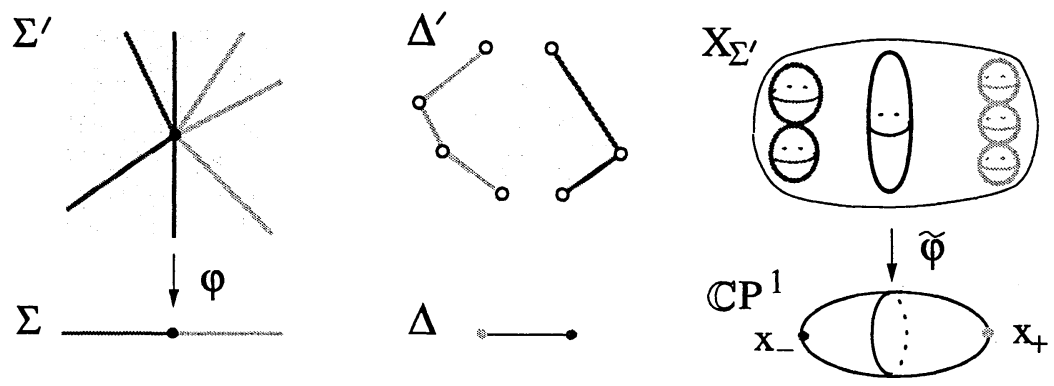

Figure 6: Figure 2-2-1. A toric morphism $\widetilde{\varphi}: X_{\Sigma^{\prime}} \rightarrow \mathbb{C} P^{1}$ and its generic and the two special fibers are indicated. The primitive faces of $\Delta^{\prime}$ are darkened/shaded.

It is worth emphasizing that in this case the fiber over $x_{ \pm}$is a union of codimension1 orbit closures in $X_{\Sigma^{\prime}}$; in other words, it is a T-Weil divisor in $X_{\Sigma^{\prime}}$.

Example 2.2.2 [B-C-dlO-G]. In [B-C-dlO-G], Braun, Candelas, de la Ossa, and Grassi consider a 5-dimensional toric variety associated to a reflexive polytope, in which the Calabi-Yau hypersurfaces admit an elliptic fibration. In their work, they characterize the existence of the fibration and the generic fiber at the level of toric varieties and study also the gauge group of the effective 4-dimensional supersymmetric field theory and the divisors of the Calabi-Yau hypersurfaces that contribute to the superpotential of that effective field theory. Here let us study their example of toric fibration in more details. (The restriction of this fibration to Calabi-Yau hypersurfaces will be studied in detail in Example 4.2.1 in Sec. 4.2.) To make the comparison with their work easier, we shall follow their notations in describing the fan $\Sigma^{\prime}$ with modifications to fit into our earlier notations. The notations for the fan $\Sigma$ are modified from [Ra].

(a) The toric data of $X_{\Sigma^{\prime}}$ and $X_{\Sigma}$. The 1-skeleton of the fans and the labels of the 1 -rays are given in the following table: 


\begin{tabular}{|r|c||c|c|}
\hline \multicolumn{2}{|c||}{$\Sigma^{\prime}(1)$} & \multicolumn{2}{|c|}{$\Sigma(1)$} \\
\hline$v_{1}^{\prime}$ & $(-1,0,0,2,3)$ & $d_{4}$ & $(-1,0,0)$ \\
$v_{2}^{\prime}$ & $(0,-1,0,2,3)$ & $d_{3}$ & $(0,-1,0)$ \\
$c_{1}^{\prime}$ & $(0,0,-1,2,3)$ & $r_{2}$ & $(0,0,-1)$ \\
$c_{2}^{\prime}$ & $(0,0,-1,1,2)$ & $r_{1}$ & $(0,0,1)$ \\
$v_{4}^{\prime}$ & $(0,0,0,-1,0)$ & $d_{2}$ & $(0,1,2)$ \\
$v_{5}^{\prime}$ & $(0,0,0,0,-1)$ & $u$ & $(0,1,3)$ \\
$b^{\prime}$ & $(0,0,0,2,3)$ & $d_{1}$ & $(1,0,4)$ \\
$e_{1}^{\prime}$ & $(0,0,1,2,3)$ & & \\
$e_{2}^{\prime}$ & $(0,0,2,2,3)$ & & \\
$e_{3}^{\prime}$ & $(0,0,1,1,1)$ & & \\
$f^{\prime}$ & $(0,1,2,2,3)$ & & \\
$g^{\prime}$ & $(0,1,3,2,3)$ & & \\
$v_{6}^{\prime}$ & $(1,0,4,2,3)$ & & \\
\hline
\end{tabular}

Denote a cone e.g. $\left[v_{1}^{\prime}, b^{\prime}, e_{1}^{\prime}, v_{2}^{\prime}, v_{4}^{\prime}\right]$, generated $v_{1}^{\prime}, b^{\prime}, e_{1}^{\prime}, v_{2}^{\prime}$, and $v_{4}^{\prime}$, by $v_{1}^{\prime} b^{\prime} e_{1}^{\prime} v_{2}^{\prime} v_{4}^{\prime}$ for brevity of notation. Then the 5 -dimensional fan $\Sigma^{\prime}$ consists of 54 maximal (simplicial) cones :

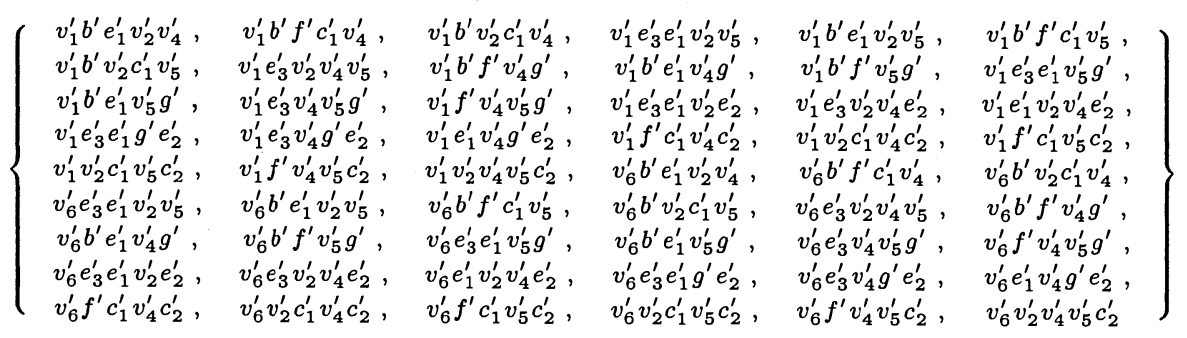

while the 3-dimensional fan $\Sigma$ consists of 10 maximal (simplicial) cones :

$\left\{d_{4} d_{2} r_{2}, d_{4} d_{2} u, d_{4} u r_{1}, d_{4} d_{3} r_{1}, d_{4} d_{3} r_{2}, d_{1} d_{2} r_{2}, d_{1} d_{2} u, d_{1} u r_{1}, d_{1} d_{3} r_{1}, d_{1} d_{3} r_{2}\right\}$.

Following [B-C-dlO-G] and denoting a fan as the summation of its maximal cones, $\Sigma^{\prime}$ can be decomposed into a combination of suspensions or extensions by extra rays :

$\Sigma^{\prime}=\left(v_{1}^{\prime}+v_{6}^{\prime}\right) \Sigma^{\prime \prime} \quad$ and $\quad \Sigma^{\prime \prime}=\left(f^{\prime}+v_{2}^{\prime}\right) \Sigma^{\prime \prime(c)}+\left(g^{\prime}+v_{2}^{\prime}\right) \Sigma^{\prime \prime(e)}+f^{\prime} g^{\prime} \Sigma^{\prime \prime}(0)$,

where

$$
\begin{aligned}
& \Sigma^{\prime \prime(c)}=b^{\prime} c_{1}^{\prime} v_{4}^{\prime}+c_{1}^{\prime} c_{2}^{\prime} v_{4}^{\prime}+b^{\prime} c_{1}^{\prime} v_{5}^{\prime}+c_{1}^{\prime} c_{2}^{\prime} v_{5}^{\prime}+c_{2}^{\prime} v_{4}^{\prime} v_{5}^{\prime}, \\
& \Sigma^{\prime \prime(e)}=e_{1}^{\prime} e_{2}^{\prime} e_{3}^{\prime}+b^{\prime} e_{1}^{\prime} v_{4}^{\prime}+e_{1}^{\prime} e_{2}^{\prime} v_{4}^{\prime}+e_{2}^{\prime} e_{3}^{\prime} v_{4}^{\prime}+b^{\prime} e_{1}^{\prime} v_{5}^{\prime}+e_{1}^{\prime} e_{3}^{\prime} v_{5}^{\prime}+e_{3}^{\prime} v_{4}^{\prime} v_{5}^{\prime}, \\
& \Sigma^{\prime \prime(0)}=b^{\prime} v_{4}^{\prime}+b^{\prime} v_{5}^{\prime}+v_{4}^{\prime} v_{5}^{\prime} .
\end{aligned}
$$

Observe that $\Sigma^{\prime \prime(0)}=\Sigma^{\prime \prime(c)} \cap \Sigma^{\prime \prime(e)}$.

Similarly, $\Sigma$ is the suspension by $\left\{d_{4}, d_{1}\right\}$ of the 2-dimensional subfan generated by $\Sigma(1)-\left\{d_{4}, d_{1}\right\}$ and can be written as

$$
\Sigma=\left(d_{4}+d_{1}\right) \Sigma^{\sim}, \text { where } \Sigma^{\sim}=\left(d_{2}+d_{3}\right) r_{2}+\left(u+d_{3}\right) r_{1}+d_{2} u .
$$


Note that all the cones in $\Sigma$ have multiplicity 1 and hence $X_{\Sigma}$ is a smooth toric 3 -fold. It is the blow-up of the Hirzebruch 3 -fold $\mathbb{F}_{024}$ at a point, cf. Table 4.1 in [Ra].

(b) The toric morphism $\tilde{\varphi}: X_{\Sigma^{\prime}} \rightarrow X_{\Sigma}$. The map of fans $\varphi: \Sigma^{\prime} \rightarrow \Sigma$ is induced by the projection map $\varphi:\left(y_{1}, y_{2}, y_{3}, y_{4}, y_{5}\right) \mapsto\left(y_{1}, y_{2}, y_{3}\right)$. Under this map,

$$
\Sigma^{\prime \prime(0)} \rightarrow 0, \quad \Sigma^{\prime \prime(c)}-\Sigma^{\prime \prime(0)} \rightarrow r_{2}, \quad \Sigma^{\prime \prime(e)}-\Sigma^{\prime \prime(0)} \rightarrow r_{1} .
$$

Together with the correspondence

$$
v_{1}^{\prime} \rightarrow d_{4}, \quad v_{6}^{\prime} \rightarrow d_{1}, \quad f^{\prime} \rightarrow d_{2}, \quad g^{\prime} \rightarrow u, \quad v_{2}^{\prime} \rightarrow d_{3},
$$

the set $\Sigma_{\sigma}^{\prime}$ of cones in $\Sigma^{\prime}$ associated to $\sigma \in \Sigma$ under $\varphi$ and the set $\Sigma_{\sigma}^{\prime \circ}$ of primitive cones therein can be directly worked out. Furthermore, from Remark 2.1.12, $\operatorname{Ind}(\sigma)=1$ for all $\sigma \in \Sigma$ since $\varphi$ is surjective. All these are listed in Columns 2 - 4 of Table 2-2-2 below. 


\begin{tabular}{|c|c|c|c|c|}
\hline$\sigma \in \Sigma$ & $\Sigma_{\sigma}^{\prime}$ & $\tau^{\prime} \in \Sigma_{\sigma}^{\prime \circ}$ & $\operatorname{Ind}(\sigma)$ & the irreducible fiber component $F_{\sigma}^{\tau^{\prime}}$ \\
\hline 0 & $\varphi^{-1}(0)=\Sigma^{\prime \prime(0)}$ & 0 & 1 & $\mathrm{~W} \mathbb{C P}^{2}(1,2,3)$ \\
\hline$d_{4}$ & $v_{1}^{\prime} \Sigma^{\prime \prime(0)}$ & $v_{1}^{\prime}$ & 1 & $\mathrm{~W} \mathbb{C P}^{2}(1,2,3)$ \\
\hline$d_{3}$ & $v_{2}^{\prime} \Sigma^{\prime \prime(0)}$ & $v_{2}^{\prime}$ & 1 & $\mathrm{~W} \overline{\mathbb{C P}^{2}(1,2,3)}$ \\
\hline$r_{2}$ & $\Sigma^{\prime \prime(c)}-\Sigma^{\prime \prime(0)}$ & $c_{1}^{\prime}, c_{2}^{\prime}$ & 1 & $X(4), \quad \mathbb{C P}^{2}$ \\
\hline$r_{1}$ & $\Sigma^{\prime \prime(e)}-\Sigma^{\prime \prime(0)}$ & $e_{1}^{\prime}, e_{2}^{\prime}, e_{3}^{\prime}$ & 1 & $X(5), \quad \mathrm{W} \mathbb{C P}^{2}(1,1,3), \quad \mathbb{F}_{2}$ \\
\hline$d_{2}$ & $f^{\prime} \Sigma^{\prime \prime(0)}$ & & 1 & $\mathrm{~W} \mathbb{C P} P^{2}(1,2,3)$ \\
\hline$u$ & $g^{\prime} \Sigma^{\prime \prime(0)}$ & $g^{\prime}$ & 1 & $\mathrm{~W} \mathbb{C P}^{2}(1,2,3)$ \\
\hline$d_{1}$ & $v_{6}^{\prime} \Sigma^{\prime \prime}(0)$ & $v_{6}^{\prime}$ & 1 & $\mathrm{~W} \mathbb{C P}^{2}(1,2,3)$ \\
\hline $\begin{array}{l}d_{2} r_{2} \\
d_{2} u \\
u r_{1} \\
d_{3} r_{1} \\
d_{3} r_{2} \\
\end{array}$ & $\begin{array}{l}f^{\prime}\left(\Sigma^{\prime \prime(c)}-\Sigma^{\prime \prime(0)}\right) \\
f^{\prime} g^{\prime} \Sigma^{\prime \prime(0)} \\
g^{\prime}\left(\Sigma^{\prime \prime(e)}-\Sigma^{\prime \prime(0)}\right) \\
v_{2}^{\prime}\left(\Sigma^{\prime \prime(e)}-\Sigma^{\prime \prime(0)}\right) \\
v_{2}^{\prime}\left(\Sigma^{\prime \prime(c)}-\Sigma^{\prime \prime(0)}\right)\end{array}$ & $\begin{array}{ll}f^{\prime} c_{1}^{\prime}, & f^{\prime} c_{2}^{\prime} \\
f^{\prime} g^{\prime} & \\
g^{\prime} e_{1}^{\prime}, & g^{\prime} e_{2}^{\prime}, \quad g^{\prime} e_{3}^{\prime} \\
v_{2}^{\prime} e_{1}^{\prime}, & v_{2}^{\prime} e_{2}^{\prime}, \quad v_{2}^{\prime} e_{3}^{\prime} \\
v_{2}^{\prime} c_{1}^{\prime}, & v_{2}^{\prime} c_{2}^{\prime}\end{array}$ & $\begin{array}{l}1 \\
1 \\
1 \\
1 \\
1\end{array}$ & $\begin{array}{lll}X(4), & \mathbb{C P}^{2} & \\
\mathrm{~W} \mathbb{C P}^{2}(1,2,3) & \\
X(5), \quad \mathbb{W C P}^{2}(1,1,3), & \mathbb{F}_{2} \\
X(5), & \mathrm{WCP}^{2}(1,1,3), & \mathbb{F}_{2} \\
X(4), & \mathbb{C P}^{2} & \end{array}$ \\
\hline $\begin{array}{r}d_{4} d_{3} \\
d_{4} r_{2} \\
d_{4} r_{1} \\
d_{4} d_{2} \\
d_{4} u\end{array}$ & $\begin{array}{l}v_{1}^{\prime} v_{2}^{\prime} \Sigma^{\prime \prime(0)} \\
v_{1}^{\prime}\left(\Sigma^{\prime \prime(c)}-\Sigma^{\prime \prime(0)}\right) \\
v_{1}^{\prime}\left(\Sigma^{\prime \prime(e)}-\Sigma^{\prime \prime(0)}\right) \\
v_{1}^{\prime} f^{\prime} \Sigma^{\prime \prime(0)} \\
v_{1}^{\prime} g^{\prime} \Sigma^{\prime \prime(0)}\end{array}$ & $\begin{array}{ll}v_{1}^{\prime} v_{2}^{\prime} & \\
v_{1}^{\prime} c_{1}^{\prime}, & v_{1}^{\prime} c_{2}^{\prime} \\
v_{1}^{\prime} e_{1}^{\prime}, & v_{1}^{\prime} e_{2}^{\prime}, \quad v_{1}^{\prime} e_{3}^{\prime} \\
v_{1}^{\prime} f^{\prime} & \end{array}$ & $\begin{array}{l}1 \\
1 \\
1 \\
1 \\
1\end{array}$ & $\begin{array}{l}\mathrm{W} \mathbb{C P}^{2}(1,2,3) \\
X(4), \quad \mathbb{C P}^{2} \\
X(5), \quad \mathbb{W C P}^{2}(1,1,3), \quad \mathbb{F}_{2} \\
\mathrm{~W} \mathbb{C P}^{2}(1,2,3) \\
\mathrm{W} C P^{2}(1,2,3)\end{array}$ \\
\hline $\begin{array}{r}d_{1} d_{3} \\
d_{1} r_{2} \\
d_{1} r_{1} \\
d_{1} d_{2} \\
d_{1} u \\
\end{array}$ & $\begin{array}{l}v_{6}^{\prime} v_{2}^{\prime} \Sigma^{\prime \prime}(0) \\
v_{6}^{\prime}\left(\Sigma^{\prime \prime}(c)-\Sigma^{\prime \prime}(0)\right. \\
v_{6}^{\prime}\left(\Sigma^{\prime \prime(e)}-\Sigma^{\prime \prime(0)}\right) \\
v_{6}^{\prime} f^{\prime} \Sigma^{\prime \prime(0)} \\
v_{6}^{\prime} g^{\prime} \Sigma^{\prime \prime(0)}\end{array}$ & $\begin{array}{l}v_{6}^{\prime} v_{2}^{\prime} \\
v_{6}^{\prime} c_{1}^{\prime}, v_{6}^{\prime} c_{2}^{\prime} \\
v_{6}^{\prime} e_{1}^{\prime}, \quad v_{6}^{\prime} e_{2}^{\prime}, \quad v_{6}^{\prime} e_{3}^{\prime} \\
v_{6}^{\prime} f^{\prime} \\
v_{6}^{\prime} g^{\prime}\end{array}$ & $\begin{array}{l}1 \\
1 \\
1 \\
1 \\
1\end{array}$ & $\begin{array}{l}\mathrm{W} \mathbb{C P}^{2}(1,2,3) \\
X(4), \quad \mathbb{C P}^{2} \\
X(5), \quad \mathbb{W C P}^{2}(1,1,3), \quad \mathbb{F}_{2} \\
\mathrm{~W} \mathbb{C P}^{2}(1,2,3) \\
\mathrm{WCP}^{2}(1,2,3)\end{array}$ \\
\hline $\begin{array}{l}d_{4} d_{2} r_{2} \\
d_{4} d_{2} u \\
d_{4} u r_{1} \\
d_{4} d_{3} r_{1} \\
d_{4} d_{3} r_{2}\end{array}$ & $\begin{array}{l}v_{1}^{\prime} f^{\prime}\left(\Sigma^{\prime \prime(c)}-\Sigma^{\prime \prime(0)}\right) \\
v_{1}^{\prime} f^{\prime} g^{\prime} \Sigma^{\prime \prime(0)} \\
v_{1}^{\prime} g^{\prime}\left(\Sigma^{\prime \prime(e)}-\Sigma^{\prime \prime(0)}\right) \\
v_{1}^{\prime} v_{2}^{\prime}\left(\Sigma^{\prime \prime(e)}-\Sigma^{\prime \prime(0)}\right) \\
v_{1}^{\prime} v_{2}^{\prime}\left(\Sigma^{\prime \prime(c)}-\Sigma^{\prime \prime(0)}\right)\end{array}$ & $\begin{array}{l}v_{1}^{\prime} f^{\prime} c_{1}^{\prime}, \quad v_{1}^{\prime} f^{\prime} c_{2}^{\prime} \\
v_{1}^{\prime} f^{\prime} g^{\prime} \\
v_{1}^{\prime} g^{\prime} e_{1}^{\prime}, \quad v_{1}^{\prime} g^{\prime} e_{2}^{\prime}, \quad v_{1}^{\prime} g^{\prime} e_{3}^{\prime} \\
v_{1}^{\prime} v_{2}^{\prime} e_{1}^{\prime}, \quad v_{1}^{\prime} v_{2}^{\prime} e_{2}^{\prime}, \quad v_{1}^{\prime} v_{2}^{\prime} e_{3}^{\prime} \\
v_{1}^{\prime} v_{2}^{\prime} c_{1}^{\prime}, \quad v_{1}^{\prime} v_{2}^{\prime} c_{2}^{\prime}\end{array}$ & $\begin{array}{l}1 \\
1 \\
1 \\
1 \\
1\end{array}$ & $\begin{array}{lll}X(4), & \mathbb{C P}^{2} & \\
\mathrm{~W} \mathbb{C P}^{2}(1,2,3) & \\
X(5), \quad \mathrm{W} \mathbb{C P}^{2}(1,1,3), & \mathbb{F}_{2} \\
X(5), \quad \mathbb{C P}^{2}(1,1,3), & \mathbb{F}_{2} \\
X(4), & \mathbb{C P}^{2}\end{array}$ \\
\hline $\begin{array}{l}d_{1} d_{2} r_{2} \\
d_{1} d_{2} u \\
d_{1} u r_{1} \\
d_{1} d_{3} r_{1} \\
d_{1} d_{3} r_{2}\end{array}$ & $\begin{array}{l}v_{6}^{\prime} f^{\prime}\left(\Sigma^{\prime \prime(c)}-\Sigma^{\prime \prime(0)}\right) \\
v_{6}^{\prime} f^{\prime} g^{\prime} \Sigma^{\prime \prime(0)} \\
v_{6}^{\prime} g^{\prime}\left(\Sigma^{\prime \prime(e)}-\Sigma^{\prime \prime(0)}\right) \\
v_{6}^{\prime} v_{2}^{\prime}\left(\Sigma^{\prime \prime(e)}-\Sigma^{\prime \prime(0)}\right) \\
v_{6}^{\prime} v_{2}^{\prime}\left(\Sigma^{\prime \prime(c)}-\Sigma^{\prime \prime(0)}\right)\end{array}$ & $\begin{array}{l}v_{6}^{\prime} f^{\prime} c_{1}^{\prime}, \quad v_{6}^{\prime} f^{\prime} c_{2}^{\prime} \\
v_{6}^{\prime} f^{\prime} g^{\prime} \\
v_{6}^{\prime} g^{\prime} e_{1}^{\prime}, \quad v_{6}^{\prime} g^{\prime} e_{2}^{\prime}, \quad v_{6}^{\prime} g^{\prime} e_{3}^{\prime} \\
v_{6}^{\prime} v_{2}^{\prime} e_{1}^{\prime}, \quad v_{6}^{\prime} v_{2}^{\prime} e_{2}^{\prime}, \quad v_{6}^{\prime} v_{2}^{\prime} e_{3}^{\prime} \\
v_{6}^{\prime} v_{2}^{\prime} c_{1}^{\prime}, \quad v_{6}^{\prime} v_{2}^{\prime} c_{2}^{\prime}\end{array}$ & $\begin{array}{l}1 \\
1 \\
1 \\
1 \\
1\end{array}$ & $\begin{array}{lll}X(4), & \mathbb{C P} & \\
\mathrm{W} C P^{2}(1,2,3) & \\
X(5), & \mathrm{W} \mathbb{C P}^{2}(1,1,3), & \mathbb{F}_{2} \\
X(5), & \mathbb{W C P}^{2}(1,1,3), & \mathbb{F}_{2} \\
X(4), & \mathbb{C P}^{2}\end{array}$ \\
\hline
\end{tabular}

TABLE 2-2-2. A toric description of the toric morphism $\widetilde{\varphi}$. The irreducible components $F_{\sigma}^{\tau^{\prime}}$ over the toric orbit $O_{\sigma}$ is listed in the same order as $\tau^{\prime}$. The first four columns are explained in Part (a) and Part (b); the last column is explained in Part (c), in which the notations $X(4)=X_{\{(2,3),(-1,0),(-1,-1),(0,-1)\}}$ and $X(5)=$ $X_{\{(2,3),(-1,0),(-2,-3),(-1,-2),(0,-1)\}}$ are used.

(c) The fibers of $\tilde{\varphi}$. From the table above, all the fibers of $\tilde{\varphi}$ are connected. Among the 33 torus orbits of $X_{\Sigma}$, the fiber is irreducible only over 15 of them. There are 59 primitive cones $\tau^{\prime}$ in $\Sigma^{\prime}$ with respect to the map of fans $\varphi$. To see their relative 
star $\operatorname{Star}_{\sigma}\left(\tau^{\prime}\right)$, observe that

$$
\begin{aligned}
\Sigma^{\prime \prime(c)} & =c_{1}^{\prime}\left(b^{\prime} v_{4}^{\prime}+c_{2}^{\prime} v_{4}^{\prime}+b^{\prime} v_{5}^{\prime}+c_{2}^{\prime} v_{5}^{\prime}\right)+\left(\text { cones without } c_{1}^{\prime}\right) \\
& =c_{2}^{\prime}\left(c_{1}^{\prime} v_{4}^{\prime}+c_{1}^{\prime} v_{5}^{\prime}+v_{4}^{\prime} v_{5}^{\prime}\right)+\left(\text { cones without } c_{2}^{\prime}\right) \\
& =c_{1}^{\prime} c_{2}^{\prime}\left(v_{4}^{\prime}+v_{5}^{\prime}\right)+\left(\text { cones without } c_{1}^{\prime} c_{2}^{\prime}\right) .
\end{aligned}
$$

These identities together with that fact that all the cones explicitly shown are in $\Sigma_{r_{2}}^{\prime}$ give the combinatorial type of the fans for fibers $F_{r_{2}}, F_{d_{2} r_{2}}, F_{d_{3} r_{2}}, F_{d_{4} r_{2}}, F_{d_{1} r_{2}}$, $F_{d_{4} d_{2} r_{2}}, F_{d_{4} d_{3} r_{2}}, F_{d_{1} d_{2} r_{2}}, F_{d_{1} d_{3} r_{2}}$. They imply that each of these fibers has two irreducible components, which intersect at a toric-invariant $\mathbb{C P}^{1}$. Observe also that

$$
\begin{aligned}
\Sigma^{\prime \prime(e)} & =e_{1}^{\prime}\left(e_{2}^{\prime} e_{3}^{\prime}+b^{\prime} v_{4}^{\prime}+e_{2}^{\prime} v_{4}^{\prime}+b^{\prime} v_{5}^{\prime}+e_{3}^{\prime} v_{5}^{\prime}\right)+\left(\text { cones without } e_{1}^{\prime}\right) \\
& =e_{2}^{\prime}\left(e_{1}^{\prime} e_{3}^{\prime}+e_{1}^{\prime} v_{4}^{\prime}+e_{3}^{\prime} v_{4}^{\prime}\right)+\left(\text { cones without } e_{2}^{\prime}\right) \\
& =e_{3}^{\prime}\left(e_{1}^{\prime} e_{2}^{\prime}+e_{2}^{\prime} v_{4}^{\prime}+e_{1}^{\prime} v_{5}^{\prime}+v_{4}^{\prime} v_{5}^{\prime}\right)+\left(\text { cones without } e_{3}^{\prime}\right) \\
& =e_{1}^{\prime} e_{2}^{\prime}\left(e_{3}^{\prime}+v_{4}^{\prime}\right)+\left(\text { cones without } e_{1}^{\prime} e_{2}^{\prime}\right) \\
& =e_{2}^{\prime} e_{3}^{\prime}\left(e_{1}^{\prime}+v_{4}^{\prime}\right)+\left(\text { cones without } e_{2}^{\prime} e_{3}^{\prime}\right) \\
& =e_{1}^{\prime} e_{3}^{\prime}\left(e_{2}^{\prime}+v_{5}^{\prime}\right)+\left(\text { cones without } e_{1}^{\prime} e_{3}^{\prime}\right) \\
& =e_{1}^{\prime} e_{2}^{\prime} e_{3}^{\prime}+\left(\text { cones without } e_{1}^{\prime} e_{2}^{\prime} e_{3}^{\prime}\right)
\end{aligned}
$$

These identities together with the fact that all the cones explicitly shown are in $\Sigma_{r_{1}}^{\prime}$ give the combinatorial type of the fans for fibers $F_{r_{1}}, F_{u r_{1}}, F_{d_{3} r_{1}}, F_{d_{4} r_{1}}, F_{d_{1} r_{1}}$, $F_{d_{4} u r_{1}}, F_{d_{4} d_{3} r_{1}}, F_{d_{1} u r_{1}}, F_{d_{1} d_{3} r_{1}}$. They imply also that each of these fibers has three irreducible components, any pair of which intersect at a toric-invariant $\mathbb{C P}^{1}$ and the three components intersect at a single point.

To determine $F_{\sigma}^{\tau^{\prime}}$, one needs to know the lattice structure in $\varphi^{-1}\left(\left(N_{\sigma}\right)_{\mathbb{R}}\right) /\left(N_{\tau^{\prime}}^{\prime}\right)_{\mathbb{R}}$ as well. It turns out that, in the current example, the lattice in $\varphi^{-1}\left(\left(N_{\sigma}\right)_{\mathbb{R}}\right) /\left(N_{\tau^{\prime}}^{\prime}\right)_{\mathbb{R}}$ can be canonically identified with the lattice in $\left(\varphi^{-1}(0)\right)_{\mathbb{R}}$ except for $\tau^{\prime}$ that contain $e_{2}^{\prime}$. In the latter case, the lattice structure involved can be identified with the lattice $\mathbb{Z} \oplus \frac{1}{2} \mathbb{Z}$ in $\left(\varphi^{-1}(0)\right)_{\mathbb{R}}$ when an appropriate basis for the lattice $\varphi^{-1}(0)$ is chosen. One can also check that $F_{\sigma}^{\tau^{\prime}}$ depends only on which or none of $c_{1}^{\prime}, c_{2}^{\prime}, e_{1}^{\prime}, e_{2}^{\prime}, e_{3}^{\prime}$ is contained in $\tau^{\prime}$.

All these observations together with some explicit calculations give us a complete description of all the irreducible components $F_{\sigma}^{\tau^{\prime}}$ of $F_{\sigma}$ and their intersections in $F_{\sigma}$, as listed in the last column of the Table 2-2-2 and indicated in FIGURE 2-2-3 via fans. In particular, there are 6 different kinds of toric varieties that an irreducible fiber components $F_{\sigma}^{\tau^{\prime}}$ can assume.

\begin{tabular}{|l|cccccc|}
\hline ray involved & $\bullet$ & $c_{1}^{\prime}$ & $c_{2}^{\prime}$ & $e_{1}^{\prime}$ & $e_{2}^{\prime}$ & $e_{3}^{\prime}$ \\
\hline toric variety & $\mathrm{WCP}^{2}(1,2,3)$ & $X(4)$ & $\mathbb{C P}^{2}$ & $X(5)$ & $\mathrm{WCCP}^{2}(1,1,3)$ & $\mathbb{F}_{2}$ \\
\hline reflexivity & yes & yes & yes & no & no & yes \\
\hline
\end{tabular}

where $\mathrm{W} \mathbb{C P}^{2}(1,2,3)$ and $\mathrm{W} \mathbb{C P}^{2}(1,1,3)$ are weighted projective spaces, $X(4)$ (resp. 
$\left.X(5), \mathbb{F}_{2}\right)$ is the toric variety associated to the 2-dimensional fan determined by the set of 1-cones $\{(2,3),(-1,0),(-1,-1),(0,-1)\}$

(resp. $\{(2,3),(-1,0),(-2,-3),(-1,-2),(0,-1)\},\{(1,0),(0,1),(-1,2),(0,-1)\})$.

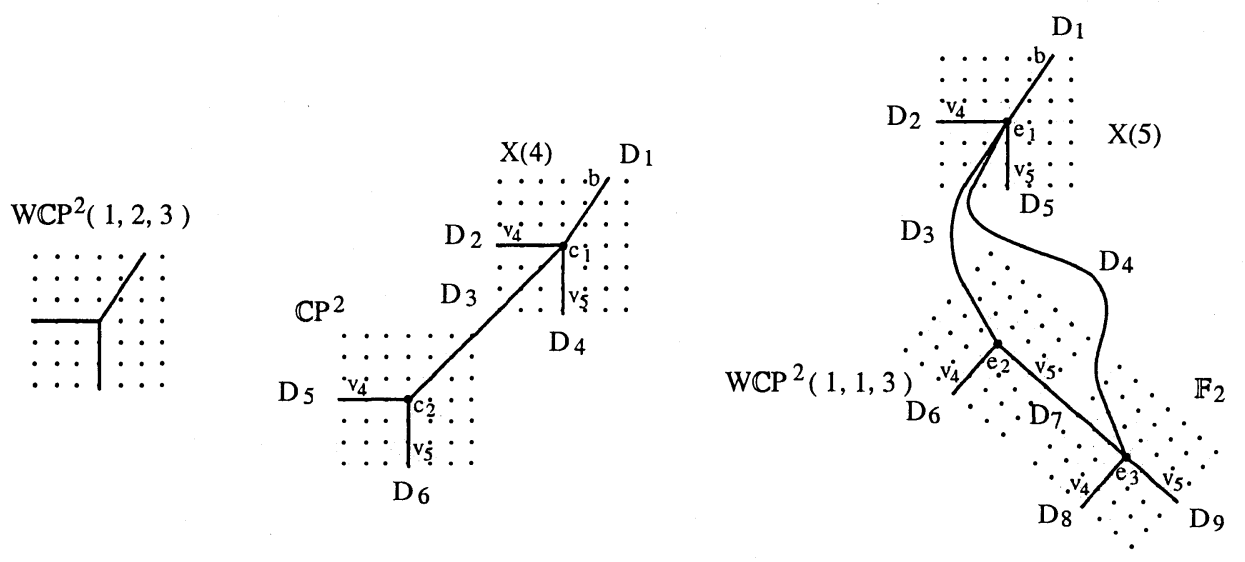

Figure 2-2-3. The fans for the generic fiber and the reducible nongeneric fibers are indicated. An reducible toric variety can be coded in a multi-fan; the intersection relations of irreducible components can also be read off from it. The 1-cones in $\Sigma^{\prime}(1)$ that give rise to the corresponding cones for the fiber are used as labels. The T-Weil diviser $D_{i}$ associated to each ray is also indicated for later use. (For clarity of picture, all the "'"'s are omitted.)

Remark 2.2.3. Note that in Example 2.2.2, each non-degenerate fiber contains an irreducible component that is associated to a reflexive polytope (while it may have other components that are not). It is interesting to know if this is always so for toric morphisms from fans related to reflexive polytopes. If not, then how to characterize those fibrations that have this property.

\section{Induced morphism and fibers for hypersurfaces.}

Having understood the fibers of a toric morphism $\tilde{\varphi}: X_{\Sigma^{\prime}} \rightarrow X_{\Sigma}$, our next theme is the study of the induced morphism $\left.\tilde{\varphi}\right|_{Y^{\prime}}: Y^{\prime} \rightarrow X_{\Sigma}$ for a hypersurface $Y^{\prime}$ in $X_{\Sigma^{\prime}}$. Since hypersurfaces in $X_{\Sigma^{\prime}}$ that we will be interested in are realized as the zero locus of sections of some line bundle over $X_{\Sigma^{\prime}}$, the problem can be converted to the study of the various restrictions of a line bundle over $X_{\Sigma^{\prime}}$ with a section. The general study of this is given in this section. The results presented here will then be applied to the case of Calabi-Yau hypersurfaces in Sec. 4. 


\subsection{Preparations.}

We give first some basic lemmas relating $\widetilde{\varphi}$ and sections of a line bundle $\mathcal{L}$ over $X_{\Sigma^{\prime}}$ and then use the flattening stratification of $\tilde{\varphi}$ described in Proposition 2.1.4 in Sec. 2.1 to study $\left.\tilde{\varphi}\right|_{Y^{\prime}}$ for $Y^{\prime}$ realized as the zero-locus of a section of $\mathcal{L}$.

Recall first the following facts: (Proposition 2.4 and Corollary 2.5 in [Od2])

Fact 3.1.1 [equivariant line bundle]. Let $X^{\prime}=X_{\Sigma^{\prime}}, S F\left(\Sigma^{\prime}\right)$ be the group of continuous functions from $N_{\mathbb{R}}^{\prime}$ to $\mathbb{R}$ that are linear on each $\sigma^{\prime} \in \Sigma^{\prime}$, and $C D i v_{T_{N^{\prime}}}\left(X^{\prime}\right)$ be the group of $T_{N^{\prime}}$-invariant Cartier divisors on $X$. For $h^{\prime} \in S F\left(\Sigma^{\prime}\right)$, let $D_{h^{\prime}}$ be the Cartier divisor on $X^{\prime}$ associated to $h^{\prime}$. Then

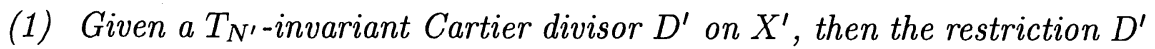
to each affine open set $U_{\sigma^{\prime}}, \sigma^{\prime} \in \Sigma^{\prime}$, coincides with a principal divisor on $U_{\sigma^{\prime}}$ so that $D^{\prime}=D_{h^{\prime}}$ for some $h^{\prime} \in S F\left(\Sigma^{\prime}\right)$. The map $h^{\prime} \mapsto D_{h^{\prime}}$ gives an isomorphism $S F\left(\Sigma^{\prime}\right) \stackrel{\sim}{\rightarrow} C D_{i v} v_{T_{N^{\prime}}}\left(X^{\prime}\right)$.

(2) For any Cartier divisor $D^{\prime}$ on $X^{\prime}$, one has an $\mathcal{O}_{X^{\prime}}$-module isomorphism $\mathcal{O}_{X^{\prime}}\left(D^{\prime}\right) \simeq \mathcal{O}_{X^{\prime}}\left(D_{h^{\prime}}\right)$ for some $h^{\prime} \in S F\left(\Sigma^{\prime}\right)$. Thus, the composition of natural homomorphisms $S F\left(\Sigma^{\prime}\right) \rightarrow C D i v\left(X^{\prime}\right) \rightarrow \operatorname{Pic}\left(X^{\prime}\right)$ is surjective.

(3) The following are equivalent for $h^{\prime} \in S F\left(\Sigma^{\prime}\right)$ :

(a) $h^{\prime} \in M^{\prime}$.

(b) $D_{h^{\prime}}$ is a principal divisor.

(c) $\mathcal{O}_{X^{\prime}}\left(D_{h^{\prime}}\right) \simeq \mathcal{O}_{X^{\prime}}$ as $\mathcal{O}_{X^{\prime}}$-modules.

(4) As a corollary of (1), (2), and (3), one has the canonical isomorphism

$$
\begin{aligned}
S F\left(\Sigma^{\prime}\right) / M^{\prime} & \stackrel{\sim}{\rightarrow} \operatorname{CDiv}_{T_{N^{\prime}}}\left(X^{\prime}\right) / C \operatorname{Civ}_{T_{N^{\prime}}}\left(X^{\prime}\right) \cap P \operatorname{Div}\left(X^{\prime}\right) \\
& \stackrel{\sim}{ } \operatorname{ELB}\left(X^{\prime}\right) /\left\{\mathcal{O}_{X^{\prime}}\left(m^{\prime}\right) \mid m^{\prime} \in M^{\prime}\right\} \stackrel{\sim}{\rightarrow} \operatorname{Pic}\left(X^{\prime}\right),
\end{aligned}
$$

where PDiv $\left(X^{\prime}\right)$ is the group of principal divisors on $X^{\prime}, E L B\left(X^{\prime}\right)$ is the group of equivariant line bundles over $X^{\prime}$, and $\mathcal{O}_{X^{\prime}}\left(m^{\prime}\right)$ is the equivariant trivial line bundle $\mathcal{O}_{X^{\prime}}$ with the linearization given by the character $\chi^{m^{\prime}}$.

Remark 3.1.2 ([Fu] and [Od2], also [Ka]). An element $h^{\prime} \in S F\left(\Sigma^{\prime}\right)$ determines (non-uniquely) a system of weights $W_{h^{\prime}}=\left\{m_{\sigma^{\prime}}^{\prime} \in M^{\prime} \mid \sigma^{\prime} \in \Sigma^{\prime}\right\}$, from which the following data are specified:

(1) The linearization for the trivialization of $\mathcal{L}_{h^{\prime}}$ when restricted to the affine charts $U_{\sigma^{\prime}}$ for $X_{\Sigma^{\prime}}$. Explicitly, this is given by $t^{\prime} \cdot\left(x^{\prime}, a\right)=\left(t^{\prime}\right.$. $\left.x^{\prime}, \chi^{m_{\sigma^{\prime}}}\left(t^{\prime}\right) a\right)$, where $t^{\prime} \in T_{N^{\prime}}$ and $\left(x^{\prime}, a\right) \in U_{\sigma^{\prime}} \times \mathbb{C}$.

(2) The transition function between local trivializations: $f_{\sigma_{1}^{\prime} \sigma_{2}^{\prime}}: U_{\sigma_{1}^{\prime}} \cap U_{\sigma_{2}^{\prime}} \rightarrow$

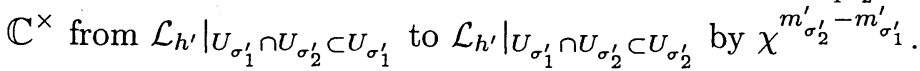

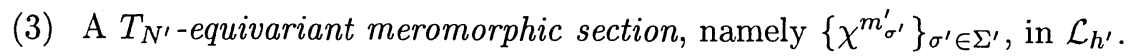


The weights $\left\{m_{\sigma^{\prime}}^{\prime} \in M^{\prime} \mid \sigma^{\prime} \in \Sigma^{\prime}\left(n^{\prime}\right)\right\}$ determines an integral polytope $\Delta_{h^{\prime}}$ in $M_{\mathbb{R}}^{\prime}$ by taking the convex hull and one has

$$
\begin{array}{ccc}
\oplus_{m^{\prime} \in \Delta_{h^{\prime}} \cap M^{\prime}} \mathbb{C} \cdot \chi^{m^{\prime}} & \simeq & H^{0}\left(X_{\Sigma^{\prime}}, \mathcal{L}_{h^{\prime}}\right) \\
m^{\prime} & \mapsto & s_{m^{\prime}}
\end{array},
$$

where $s_{m^{\prime}}=\chi^{m^{\prime}-m_{\sigma^{\prime}}^{\prime}}$ over each affine chart $U_{\sigma^{\prime}}, \sigma^{\prime} \in \Sigma^{\prime}$. The compatibility of elements in $W_{h^{\prime}}$ implies that $\Sigma^{\prime}$ is a refinement of the normal fan of $\Delta_{h^{\prime}}$.


with the linearization of $\mathcal{O}_{X^{\prime}}\left(D_{h^{\prime}}\right)$ determined by the weight system $W_{h^{\prime}}$ in Remark

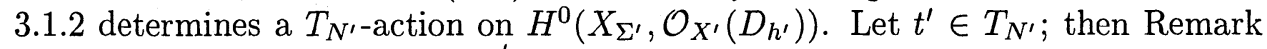
3.1.2 implies that $t^{\prime} \cdot s_{m^{\prime}}=\chi^{-m^{\prime}}\left(t^{\prime}\right) \cdot s_{m^{\prime}}$ over $U_{\sigma^{\prime}}$ for $m^{\prime} \in \Delta^{\prime} \cap M^{\prime}$.

Fact 3.1.4 [restriction of line bundle to orbit closure]. (Cf. [Fu].) Given a line bundle $\mathcal{L}_{\Delta^{\prime}}$ over $X_{\Sigma^{\prime}}$. Let $h^{\prime} \in S F\left(\Sigma^{\prime}\right)$ be the associated continuous piecewise linear function on $N_{\mathbb{R}}^{\prime}$ with respect to $\Sigma^{\prime}$. Then the restriction of $\mathcal{L}_{\Delta^{\prime}}$ to an orbit closure $V\left(\tau^{\prime}\right)$ can be described as follows:

(1) Let $m_{\tau^{\prime}}^{\prime} \in M^{\prime}$ be a linear function on $N_{\mathbb{R}}^{\prime}$ such that $\left.m_{\tau^{\prime}}^{\prime}\right|_{\tau^{\prime}}=\left.h^{\prime}\right|_{\tau^{\prime}}$. Then $h^{\prime \prime}=h^{\prime}-m_{\tau^{\prime}}^{\prime} \in S F\left(\Sigma^{\prime}\right)$ defines a line bundle $\mathcal{L}_{h^{\prime \prime}}$ isomorphic to $\mathcal{L}_{h^{\prime}}$ (with different linearization). $\mathcal{L}_{h^{\prime \prime}}$ corresponds to the polytope $\Delta^{\prime}-m_{\tau^{\prime}}^{\prime}$.

(2) After this shift, the restriction of $h^{\prime \prime}$ to the set of cones $\sigma^{\prime}$ with $\tau^{\prime} \prec \sigma^{\prime}$ descends to a continuous piecewise linear function on $\left(N^{\prime} / N_{\sigma^{\prime}}^{\prime}\right)_{\mathbb{R}}$ with respect to Star $\left(\tau^{\prime}\right)$. This defines a line bundle over $V\left(\tau^{\prime}\right)$ isomorphic to $\left.\mathcal{L}_{\Delta^{\prime}}\right|_{V\left(\tau^{\prime}\right)}$ (with perhaps different linearization).

(3) In terms of polytopes, let $\sigma_{i}^{\prime}, i=1, \ldots, k$, be the maximal cones in $\Sigma^{\prime}$ with $\tau^{\prime} \prec \sigma_{i}^{\prime}$. Let $m_{i}^{\prime}$ be the vertices of $\Delta^{\prime}$ corresponding to the restriction $\left.h^{\prime}\right|_{\sigma_{i}^{\prime}}$. Since all $m_{i}^{\prime}$ give the same restriction to $\tau^{\prime}$, all the differences $m_{i}^{\prime}-m_{j}^{\prime}$ lie in sublattice $\tau^{\perp \perp} \cap M^{\prime}$. The convex hull $\Delta_{\tau^{\prime}}^{\prime}$ of $\left\{m_{1}^{\prime}, \ldots, m_{k}^{\prime}\right\}$ in $M_{\mathbb{R}}^{\prime}$ is then contained in, say, $m_{1}^{\prime}+\tau^{\prime \perp}$ and hence determines an integral polytope in $\tau^{\perp}$, unique up to a translation by a lattice point. This is the polytope in $\tau^{\prime \perp}$ that gives $\left.\mathcal{L}_{\Delta^{\prime}}\right|_{V\left(\tau^{\prime}\right)}$, up to a linearization.

The following lemma characterizes $\Delta_{\tau^{\prime}}^{\prime}$ up to a translation.

Lemma 3.1.5. $\Delta_{\tau^{\prime}}^{\prime}=\left(\Delta^{\prime}-m_{\tau^{\prime}}^{\prime}\right) \cap \tau^{\prime \perp}$ up to an integral translation.

Proof. After the translation, $\Delta_{\tau^{\prime}}^{\prime}$ becomes the convex hull of $\left\{m_{1}^{\prime}-m_{\tau^{\prime}}^{\prime}, \ldots, m_{k}^{\prime}-\right.$ $\left.m_{\tau^{\prime}}^{\prime}\right\}$ contained in $\left(\Delta^{\prime}-m_{\tau^{\prime}}^{\prime}\right) \cap \tau^{\prime \perp}$. If there is an extra integral point $m_{0}^{\prime}$ in $\left(\Delta^{\prime}-m_{\tau^{\prime}}^{\prime}\right) \cap \tau^{\prime \perp}$ besides those in $\Delta_{\tau^{\prime}}^{\prime}$, then, since $m_{0}^{\prime} \in\left(\Delta^{\prime}-m_{\tau^{\prime}}^{\prime}\right)$, it gives rise to a holomorphic section in $\mathcal{L}=\mathcal{L}_{\Delta^{\prime}-m_{\tau^{\prime}}^{\prime}}$ and hence on $\left.\mathcal{L}\right|_{V\left(\tau^{\prime}\right)}$. On the other hand, since 
it is outside $\Delta_{\tau^{\prime}}^{\prime}$, it cannot be holomorphic on $V\left(\tau^{\prime}\right)$. This leads to a contradiction and one concludes the lemma.

Consider now the restriction homomorphism

$$
\text { res : } H^{0}\left(X_{\Sigma^{\prime}}, \mathcal{L}_{\Delta^{\prime}}\right) \longrightarrow H^{0}\left(V\left(\tau^{\prime}\right), \mathcal{L}_{\Delta_{\tau^{\prime}}^{\prime}}\right)
$$

that sends a holomorphic section of $\mathcal{L}_{\Delta^{\prime}}$ to its restriction over $V\left(\sigma^{\prime}\right)$. Note that for $m^{\prime} \in\left(\Delta^{\prime} \cap M^{\prime}\right)-\Delta_{\tau^{\prime}}^{\prime}$, the associated holomorphic section in $\mathcal{L}_{\Delta^{\prime}}$ restricted to $V\left(\tau^{\prime}\right)$ gives the 0 -section in $\mathcal{L}_{\Delta_{\tau^{\prime}}^{\prime}}$. The lemma below follows immediately from Remark 3.1.2:

Lemma 3.1.6 [sections restricted to orbit closure]. The restriction homomorphism res : $H^{0}\left(X_{\Sigma^{\prime}}, \mathcal{L}_{\Delta^{\prime}}\right) \rightarrow H^{0}\left(V\left(\sigma^{\prime}\right), \mathcal{L}_{\Delta_{\tau^{\prime}}^{\prime}}\right)$ is surjective with kernel generated by the sections in $\mathcal{L}_{\Delta^{\prime}}$ associated to $\Delta^{\prime} \cap M^{\prime}{ }^{\tau^{\prime}} \Delta_{\tau^{\prime}}^{\prime}$. The restriction to $V\left(\tau^{\prime}\right)$ of the section $s_{m^{\prime}}$ in $\mathcal{L}_{\Delta^{\prime}}$ associated to $m^{\prime} \in \Delta_{\tau^{\prime}}^{\prime} \cap M^{\prime} \subset \Delta^{\prime}$ is the section in $\mathcal{L}_{\Delta_{\tau^{\prime}}^{\prime}}$ given by the same $m^{\prime} \in \Delta_{\tau^{\prime}}^{\prime}$.

Finally, let us turn to the restriction of a line bundle to a generic fiber of a toric morphism. The following lemma characterizes pullback line bundles and sections from a toric morphism in terms of toric data:

Lemma 3.1.7 [pullback of line bundle and section]. Given lattices $N_{1}, N_{2}$, and fans $\Sigma_{i}$ in $N_{i \mathbb{R}}, i=1,2$. Let $\tilde{f}: X_{\Sigma_{1}} \rightarrow X_{\Sigma_{2}}$ be a toric morphism associated to a map of fans $f: \Sigma_{1} \rightarrow \Sigma_{2}$. Let $M_{i}$ be the dual lattice of $N_{i}$ and $f^{\dagger}: M_{2} \rightarrow M_{1}$ be the dual of $f$. Suppose that $\Delta_{2}$ is an integral polytope in $M_{2 \mathbb{R}}$. Then $\widetilde{f}^{*} \mathcal{L}_{\Delta_{2}}=\mathcal{L}_{f^{\dagger}\left(\Delta_{2}\right)}$. Furthermore, if $s$ is a section of $\mathcal{L}_{\Delta_{2}}$ that corresponds to a lattice point $m \in \Delta_{2}$, then $f^{*} s$ is the section of $f^{*} \mathcal{L}_{\Delta_{2}}$ that corresponds to $f^{\dagger}(m) \in f^{\dagger}\left(\Delta_{2}\right)$.

Proof. Let $h_{2} \in S F\left(\Sigma_{2}\right)$ be the piecewise linear function on $N_{2 \mathbb{R}}$ such that $\mathcal{L}_{h_{2}}=$ $\mathcal{L}_{\Delta_{2}}$. Then the first assertion follows from the isomorphisms $\widetilde{f}^{*} \mathcal{L}_{\Delta_{2}}=\widetilde{f}^{*} \mathcal{L}_{h_{2}}=$ $\mathcal{L}_{h_{2} \circ f}=\mathcal{L}_{f^{\dagger}\left(\Delta_{2}\right)}$ as equivariant line bundles.

For the second assertion, recall from [Od2] that the piecewise linear function $h_{2}$ determines a local linearized trivialization of $\mathcal{L}_{\Delta_{2}}$ over the affine chart $U_{\sigma_{2}}$ for each $\sigma_{2} \in \Sigma_{2}$ together with a meromorphic section up to a constant multiple. This meromorphic section over $U_{\sigma_{2}}$ is determined by the restriction $\left.h_{2}\right|_{\sigma_{2}}$, which can be represented by an $m_{\sigma_{2}} \in \Delta_{2} \cap M_{2}$. The section $s$ associated to $m_{2}$ is then realized by the character $\chi^{m_{2}-m_{\sigma_{2}}}$ over $U_{\sigma_{2}}$. Note also that, for $\sigma_{1}^{\prime} \in \Sigma_{1}$, let $m_{\sigma_{1}^{\prime}}=f^{\dagger}\left(m_{\sigma_{2}^{\prime}}\right)$, where $\sigma_{2}^{\prime}$ is the unique cone in $\Sigma_{2}$ such that the interior of $\sigma_{1}^{\prime}$ is mapped by $f$ to the interior of $\sigma_{2}^{\prime}$, then $f^{\dagger}\left(\Delta_{2}\right)$ is the convex hull of $\left\{m_{\sigma_{1}^{\prime}} \mid \sigma_{1}^{\prime} \in \Sigma_{1}\right\}$.

Now suppose that the interior of $\sigma_{1} \in \Sigma_{1}$ is mapped by $f$ to the interior of $\sigma_{2}$. Then $\widetilde{f}\left(U_{\sigma_{1}}\right) \subset U_{\sigma_{2}}$ and $\widetilde{f}^{*}\left(\left.s\right|_{U_{\sigma_{2}}}\right)$ is a holomorphic section in $\widetilde{f}^{*}\left(\left.\mathcal{L}_{\Delta_{2}}\right|_{U_{\sigma_{2}}}\right)$ 
represented by the character $\chi^{f^{\dagger}\left(m_{2}\right)-f^{\dagger}\left(m_{\sigma_{2}}\right)}$. Since the local meromorphic section of $\left.\tilde{f}^{*}\left(\mathcal{L}_{\Delta_{2}}\right)\right|_{U_{\sigma_{1}}}$ determined by $f^{\dagger}\left(\Delta_{2}\right)$ is given by $\chi^{f^{\dagger}\left(m_{\sigma_{2}}\right)}$ and this discussion holds for every $\sigma_{2} \in \Sigma_{2}$ that contains some cone in $f\left(\Sigma_{1}\right), f^{\dagger}\left(m_{2}\right)$ must correspond to $\tilde{f}^{*} s$. This concludes the proof.

Recall the toric morphism $\widetilde{\varphi}: X_{\Sigma^{\prime}} \rightarrow X_{\Sigma}$ and the notations from Sec. 2 .

Corollary 3.1.8 [line bundle and sections restricted to fiber]. Let $\mathcal{L}_{\Delta^{\prime}}$ be $a$ line bundle over $X_{\Sigma^{\prime}}$.

(a) The restriction of $\mathcal{L}_{\Delta^{\prime}}$ to the irreducible component $F_{\sigma}^{\tau^{\prime}}$ of a fiber is given by the polytope $\overline{\Delta_{\tau^{\prime}}^{\prime}}$ in $\tau^{\prime \perp} / \varphi^{\dagger}\left(\sigma^{\perp}\right)$. In particular, the (non-linearized) isomorphism class of the restriction of $\mathcal{L}_{\Delta^{\prime}}$ to each fiber over the orbit $O_{\sigma}$ in $X_{\Sigma}$ depends only on $\sigma$.

(b) The restriction to $\left.\mathcal{L}_{\Delta^{\prime}}\right|_{F_{\sigma}^{\tau^{\prime}}}$ of a section in $\mathcal{L}_{\Delta^{\prime}}$ that corresponds to $m^{\prime} \in$ $\Delta_{\tau^{\prime}} \cap M^{\prime}$ is a constant multiple of the section in $\left.\mathcal{L}_{\Delta^{\prime}}\right|_{F_{\sigma}^{\tau^{\prime}}}$ that corresponds to the lattice point $\overline{\varphi^{\dagger}}{ }_{\sigma}^{\tau^{\prime}}\left(m^{\prime}\right) \in \overline{\Delta_{\tau^{\prime}}}$.

FIGURE 3-1-1.

Proof. Since $F_{\sigma}^{\tau^{\prime}}$ is the generic fiber of the restriction of $\widetilde{\varphi}$ to the toric variety $V\left(\tau^{\prime}\right)$, without loss of generality, we only need to prove both assertions for the generic fiber of $\widetilde{\varphi}$. In this case, consider the map of fans $\varphi^{-1}(0) \rightarrow \Sigma^{\prime}$ induced by the inclusion $\operatorname{map} \tilde{\varphi}^{-1}(0) \hookrightarrow N_{\mathbb{R}}^{\prime}$. Then both assertions follow from Lemma 3.1.7.

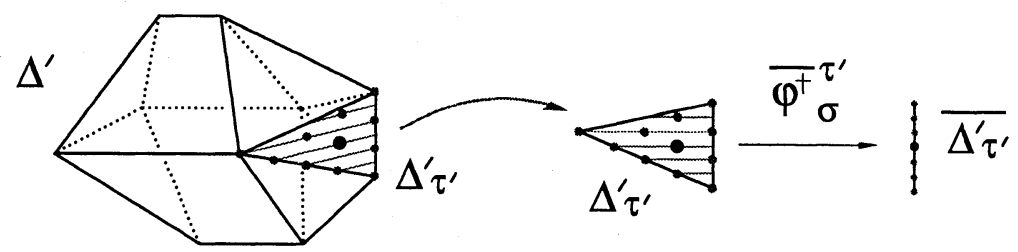

Figure 8: FiguRE 3-1-1. Restriction of a line bundle $\mathcal{L}_{\Delta^{\prime}}$ with a section $s_{m^{\prime}}$ to an irreducible fiber $F_{\sigma}^{\tau^{\prime}}$ can be understood in two steps : (1) restriction to $V\left(\tau^{\prime}\right)$, which is described by $\Delta_{\tau^{\prime}}^{\prime}$; and (2) further restriction to the generic fiber $F_{\sigma}^{\tau^{\prime}}$ of the toric morphism $\widetilde{\varphi}: V\left(\tau^{\prime}\right) \rightarrow V(\sigma)$ from the restriction of $\widetilde{\varphi}: X_{\Sigma^{\prime}} \rightarrow X_{\Sigma}$. The latter is described by the quotient $\overline{\varphi^{\dagger}} \tau_{\sigma}^{\prime}\left(m^{\prime}\right) \in \overline{\Delta_{\tau^{\prime}}^{\prime}} .\left(m^{\prime}\right.$ and its image are indicated by the thickened darkest lattice points.) 
Remark 3.1.9. When $\Sigma^{\prime}$ is the normal fan of $\Delta^{\prime}$, in which case $h^{\prime}$ is strictly convex, $\Delta_{\tau^{\prime}}^{\prime}=\Theta_{\tau^{\prime}}$, the facet of $\Delta^{\prime}$ dual to $\tau^{\prime}$.

\subsection{The induced morphism: $Y^{\prime} \subset X_{\Sigma^{\prime}} \rightarrow X_{\Sigma}$.}

Let $Y^{\prime}$ be a hypersurface of $X_{\Sigma^{\prime}}$ realized as the zero-locus of a section of $\mathcal{L}_{\Delta^{\prime}}$ and recall the flattening stratification for the toric morphism $\widetilde{\varphi}: X_{\Sigma^{\prime}} \rightarrow X_{\Sigma}$, discussed in Sec. 2.1, Proposition 2.1.4. Our strategy of understanding the induced morphism $\left.\varphi\right|_{Y^{\prime}}: Y^{\prime} \rightarrow X_{\Sigma}$ is to understand the restriction of the flattening stratification of $\tilde{\varphi}$ to $\left.\tilde{\varphi}\right|_{Y^{\prime}}$. A flattening stratification of $\left.\tilde{\varphi}\right|_{Y^{\prime}}$ with each stratum a topological bundle is then obtained by a refinement of this restriction. We discuss first a fundamental case and then all other cases, which can be reduced to the fundamental case. The notations here follow those in Sec. 2.

\section{Case (1) [fundamental] : Restriction to generic fibers for $\varphi$ surjective.}

Let $\xi: N \rightarrow N^{\prime}$ be a homomorphism such that $\varphi \circ \xi=I d_{N}$. Then $\xi$ determines a splitting of $N^{\prime}$ by

$$
\begin{array}{rlccc}
N^{\prime} & = & \operatorname{ker} \varphi+\operatorname{Im} \xi & \simeq & \operatorname{ker} \varphi \oplus N \\
v & =(v-\xi \circ \varphi(v))+\xi \circ \varphi(v) & \mapsto & (v-\xi \circ \varphi(v), \varphi(v)) .
\end{array}
$$

On the dual side, recall the quotient map $\overline{\varphi^{\dagger}}: M^{\prime} \rightarrow M^{\prime} / \varphi^{\dagger}(M)$, then $M^{\prime} / \varphi^{\dagger}(M)$ is canonically isomorphic to ker $\xi^{\dagger}$. Thus, there is a map $i_{\xi}: M^{\prime} / \varphi^{\dagger}(M) \rightarrow M^{\prime}$ such that $\xi^{\dagger} \circ i_{\xi}=0$ and that $\overline{\varphi^{\dagger}} \circ i_{\xi}=I d_{M^{\prime} / \varphi^{\dagger}(M)}$. From this, one obtains a decomposition:

$$
\begin{aligned}
& M^{\prime}=\operatorname{ker} \xi^{\dagger}+\operatorname{Im} \varphi^{\dagger} \simeq M^{\prime} / \varphi^{\dagger}(M) \oplus M \\
& \left.m^{\prime}=i_{\xi} \circ \widetilde{\varphi^{\dagger}}\left(m^{\prime}\right)+\left(m^{\prime}-i_{\xi} \circ \widetilde{\varphi^{\dagger}}\left(m^{\prime}\right)\right) \mapsto \overline{\left(\varphi^{\dagger}\right.}\left(m^{\prime}\right), \xi^{\dagger}\left(m^{\prime}\right)\right) \text {. }
\end{aligned}
$$

Note that different choices of $\xi$ give different projections to the second compoment of the decomposition $\left.\overline{\left(\varphi^{\dagger}\right.}, \xi^{\dagger}\right): M^{\prime} \stackrel{\sim}{\rightarrow} M^{\prime} / \varphi^{\dagger}(M) \oplus M$.

Let $\Delta^{\prime}$ be a rational convex polytope in $M_{\mathbb{R}}^{\prime}$ and $\mathcal{L}_{\Delta^{\prime}}$ be the associated line bundle over $X_{\Sigma^{\prime}}$. Note that, since $\xi$ in general does not induce a map of fans from $\Sigma$ to $\Sigma^{\prime}$, the polytope $\xi^{\dagger}\left(\Delta^{\prime}\right)$ in $M_{\mathbb{R}}$ may not give a line bundle over $X_{\Sigma}$. However, $\Sigma_{\xi} \doteq \tilde{\varphi}\left(\xi\left(N_{\mathbb{R}}\right) \cap \Sigma^{\prime}\right)$ is a fan in $N_{\mathbb{R}}$ that refines $\Sigma$. Thus $\xi^{\dagger}\left(\Delta^{\prime}\right)$ corresponds to the line bundle $\widetilde{\xi}^{*} \mathcal{L}_{\Delta^{\prime}}$ over $X_{\Sigma_{\xi}}$. After pushing forward by the morphism $X_{\Sigma_{\xi}} \rightarrow X_{\Sigma}$, we shall regard $\widetilde{\xi}^{*} \mathcal{L}_{\Delta^{\prime}}$ as a rank-one torsion-free sheaf over $X_{\Sigma}$.

Lemma 3.2.1 [factorization over orbit]. Let $O_{0}=T_{N}$ be the $T_{N}$-orbit in $X_{\Sigma}$ corresponding to $\{0\} \in \Sigma$ and $F_{0}$ be the fiber of $\tilde{\varphi}$ over $O_{0}$. Then: 
(a) $\xi$ determines a product decomposition $\tilde{\varphi}^{-1}\left(O_{0}\right)=O_{0} \times F_{0}$. Let $p r_{2}$ be the corresponding projection map to the second factor, then $\left.\mathcal{L}_{\Delta^{\prime}}\right|_{\widetilde{\varphi}^{-1}}\left(O_{0}\right) \simeq$ $p r_{2}^{*} \mathcal{L} \overline{\Delta^{\prime}}$.

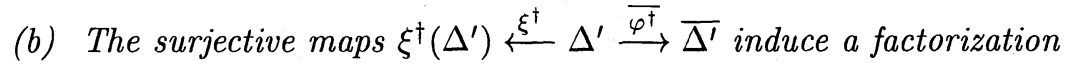

$$
\begin{array}{ccc}
H^{0}\left(\tilde{\varphi}^{-1}\left(O_{0}\right),\left.\mathcal{L}_{\Delta^{\prime}}\right|_{\tilde{\varphi}^{-1}\left(O_{0}\right)}\right) & \hookrightarrow & H^{0}\left(O_{0}, \mathcal{O}_{O_{0}}\right) \otimes H^{0}\left(F_{0}, \mathcal{L}_{\overline{\Delta^{\prime}}}\right) \\
s_{m^{\prime}} & \mapsto & s_{\xi^{\dagger}\left(m^{\prime}\right)} \otimes s \overline{\varphi^{\dagger}}\left(m^{\prime}\right)
\end{array}
$$

where $m^{\prime} \in \Delta^{\prime}$. (Here we are identifying $\mathcal{O}_{O_{0}}$ with $\left.\mathcal{L}_{\xi^{\dagger}\left(\Delta^{\prime}\right)}\right|_{O_{0}}$ implicitly.)

(c) Let $\xi_{1}, \xi_{2}: N \rightarrow N^{\prime}$ be two homomorphisms such that $\varphi \circ \xi_{i}=I d_{N}, i=$ 1, 2. Then, the $H^{0}\left(O_{0}, \mathcal{O}_{O_{0}}\right)$-factor of the factorizations in Part (b) with respect to $\xi_{1}$ and $\xi_{2}$ respectively are related by $s_{\xi_{2}^{\dagger}\left(m^{\prime}\right)}=\chi^{\left(\xi_{2}^{\dagger}-\xi_{1}^{\dagger}\right)\left(m^{\prime}\right)} s_{\xi_{1}^{\dagger}\left(m^{\prime}\right)}$ while the $H^{0}\left(F_{0}, \mathcal{L}_{\overline{\Delta^{\prime}}}\right)$-factors are the same.

Proof. As a toric variety, $\tilde{\varphi}^{-1}\left(O_{0}\right)$ is described by the fan $\Sigma_{\{0\}}^{\prime}$ in $N_{\mathbb{R}}^{\prime}$ while $F_{0}$ is described by the same fan but in the subspace $\varphi_{\mathbb{R}}^{-1}(0)$ in $N_{\mathbb{R}}^{\prime}$. The map $\xi$ induces a projection map $\pi_{\xi}: \widetilde{\varphi}^{-1}\left(O_{0}\right) \rightarrow F_{0}$ that projects the affine chart $U_{\sigma^{\prime}}$ of $\widetilde{\varphi}^{-1}\left(O_{0}\right)$

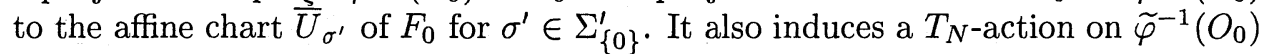
that descends under $\pi_{\xi}$ to the identity group action on $F_{0}$. Together, this specifies a decomposition $\tilde{\varphi}^{-1}\left(O_{0}\right)=O_{0} \times F_{0}$. Let $h^{\prime} \in S F\left(\Sigma^{\prime}\right)$ be a piecewise linear function on $N_{\mathbb{R}}^{\prime}$ that determines $\mathcal{L}_{\Delta^{\prime}}$. Then $\left.\mathcal{L}_{\Delta^{\prime}}\right|_{\tilde{\varphi}^{-1}\left(O_{0}\right)}$ is determined by $\left.h^{\prime}\right|_{\varphi_{\mathbb{R}}^{-1}(0)}$, whose corresponding polytope in $M^{\prime}$ can be chosen to be $i_{\xi}\left(\overline{\Delta^{\prime}}\right)$. This implies that $\left.\mathcal{L}_{\Delta^{\prime}}\right|_{\widetilde{\varphi}^{-1}\left(O_{0}\right)} \simeq p r_{2}^{*} \mathcal{L}_{\overline{\Delta^{\prime}}}$ and, hence, proves Part (a).

For Part (b), first observe that the set of affine charts $\mathcal{U}=\left\{U_{\sigma^{\prime}} \mid \sigma^{\prime} \in \Sigma_{\{0\}}^{\prime}\right\}$ forms a covering of $\tilde{\varphi}^{-1}\left(O_{0}\right)$. Over the affine chart $U_{\sigma^{\prime}}, \sigma^{\prime} \in \Sigma_{\{0\}}^{\prime}$, of $\tilde{\varphi}^{-1}\left(O_{0}\right)$, one can decompose the section $s_{m^{\prime}}$ in $\left.\mathcal{L}_{\Delta^{\prime}}\right|_{\tilde{\varphi}^{-1}\left(O_{0}\right)}$ associated to $m^{\prime} \in \Delta^{\prime} \cap M^{\prime}$ as

$\left.s_{m^{\prime}}\right|_{U_{\sigma^{\prime}}}=\chi^{m^{\prime}-m_{\sigma^{\prime}}^{\prime}}=\chi^{\xi^{\dagger}\left(m^{\prime}\right)-\xi^{\dagger}\left(m_{\sigma^{\prime}}^{\prime}\right)} \cdot \chi^{\overline{\varphi^{\dagger}}\left(m^{\prime}\right)-\overline{\varphi^{\dagger}}\left(m_{\sigma^{\prime}}^{\prime}\right)}=\chi^{\xi^{\dagger}\left(m^{\prime}\right)} \cdot \chi^{\overline{\varphi^{\dagger}}\left(m^{\prime}\right)-\overline{\varphi^{\dagger}}\left(m_{\sigma^{\prime}}^{\prime}\right)}$

since $\xi^{\dagger} \circ i_{\xi}=0$. One notes that $\chi^{\xi^{\dagger}\left(m^{\prime}\right)}$ is the section $s_{\xi^{\dagger}\left(m^{\prime}\right)}$ of $\mathcal{O}_{O_{0}}$ and $\chi^{\overline{\varphi^{\dagger}}\left(m^{\prime}\right)-\overline{\varphi^{\dagger}}\left(m_{\sigma^{\prime}}^{\prime}\right)}$ is the section $s \overline{\varphi^{\dagger}\left(m^{\prime}\right)}$ of $\mathcal{L}_{\overline{\Delta^{\prime}}}$ restricted to $\bar{U}_{\sigma^{\prime}}$. Since the transition map between the affine charts of $\widetilde{\varphi}^{-1}\left(O_{0}\right)$ in $\mathcal{U}$ is trivial along the $O_{0}$-component of the product decomposition of $\widetilde{\varphi}^{-1}\left(O_{0}\right)$, this proves Part (b).

Finally, Part (c) follows from the proof of Part (b). This concludes the proof. 
Let $\xi_{1}, \xi_{2}: N \rightarrow N^{\prime}$ be two homomorphisms such that $\varphi \circ \xi_{i}=I d_{N}, i=1,2$. Then the difference $\xi_{2}-\xi_{1}$ induces morphisms

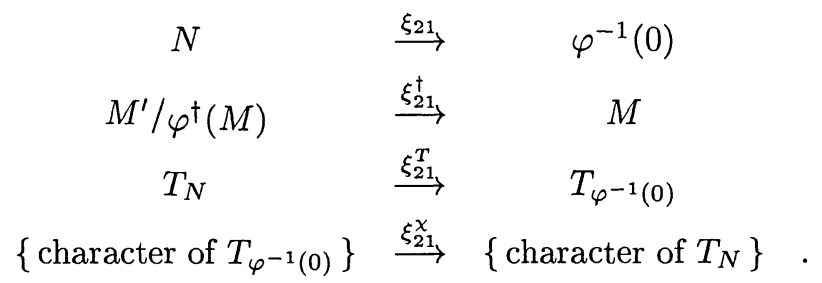

In terms of these, the transition factor $\chi^{\left(\xi_{2}^{\dagger}-\xi_{1}^{\dagger}\right)\left(m^{\prime}\right)}$ in Part (c) of Lemma 3.2.1, as a character of $T_{N}$, is simply $\xi_{21}^{\chi}\left(\chi^{\overline{\varphi^{\dagger}}\left(m^{\prime}\right)}\right)=\chi^{\overline{\varphi^{\dagger}}\left(m^{\prime}\right)} \circ \xi_{21}^{T}$. Thus, Lemma 3.2.1 and Remark 3.1.3 together imply :

Corollary 3.2.2 [torically equivalent restriction]. The description of the restriction of a section of $\mathcal{L}_{\Delta^{\prime}}$ to a fiber $F_{0}$ over $O_{0}$ by different choices of $\xi$ give rise to torically equivalent sections in $H^{0}\left(F_{0}, \mathcal{L}_{\overline{\Delta^{\prime}}}\right)$.

In particular, when one considers the hypersurfaces from the zero-locus of sections of $\mathcal{L}_{\Delta^{\prime}}$, though a $\xi$ has to be chosen to give an explicit description of its restriction to the generic fiber, the choice of $\xi$ does not change the toric equivalence class of the hypersurfaces in the fibers.

Remark 3.2.3. The situation here is pretty much like local trivialization of a bundle: There is no canonical one; however, different local trivializations are related by automorphisms of the fiber.

\section{Case (2): Restriction to exceptional fibers for $\varphi$ surjective.}

Recall from Remark 2.1.12 that, in this case, $\operatorname{Ind}(\sigma)=\left[N / N_{\sigma}: \bar{\varphi}_{\sigma}^{\sigma^{\prime}}\left(N^{\prime} / N_{\sigma^{\prime}}^{\prime}\right)\right]=1$ for any $\sigma \in \Sigma$ and $\sigma^{\prime} \in \Sigma_{\sigma}^{\prime}$. Hence, $\bar{\varphi}_{\sigma}^{\sigma^{\prime}}: N^{\prime} / N_{\sigma^{\prime}}^{\prime} \rightarrow N / N_{\sigma}$ is surjective and $\widetilde{\bar{\varphi}}_{\sigma}^{\sigma^{\prime-1}}\left(O_{\sigma}\right)=\widetilde{\varphi}^{-1}\left(O_{\sigma}\right)=O_{\sigma} \times F_{\sigma}^{c}$. Let $\tau^{\prime}$ be a primitive element in $\Sigma_{\sigma}^{\prime}$, then the orbit closure $V\left(\tau^{\prime}\right)$ of $\tau^{\prime}$ in $X_{\Sigma^{\prime}}$ is an irreducible component of the closure of $\widetilde{\varphi}^{-1}\left(O_{\sigma}\right)$ in $X_{\Sigma^{\prime}}$ and $F_{\sigma}^{\tau^{\prime}}$ is a generic fiber of the restriction $\left.\widetilde{\varphi}\right|_{V\left(\tau^{\prime}\right)}: V\left(\tau^{\prime}\right) \rightarrow V(\sigma)$. Consequently, for each pair $\left(\sigma, \tau^{\prime}\right)$, where $\sigma \in \Sigma$ and $\tau^{\prime} \in \Sigma_{\sigma}$ primitive, one can apply the discussion in Preparations to obtain the restriction of $\mathcal{L}_{\Delta^{\prime}}$ and the sections therein to $V\left(\tau^{\prime}\right)$ and then the discussions in Case (1) [fundamental] to this bundle over $V\left(\tau^{\prime}\right)$. In this way, one can write down how the generic fiber of the morphism $Y^{\prime} \cap V\left(\tau^{\prime}\right) \rightarrow V(\sigma)$ varies along $O(\sigma)$.

Since each irreducible component of a fiber of $\widetilde{\varphi}$ must appear as a generic fiber for some $\left(\sigma, \tau^{\prime}\right)$, the discussions in Case (1) and above together gives us a description of every fiber of $Y^{\prime} \rightarrow X_{\Sigma}$ orbit by orbit. 
Together this concludes the discussion for the case $\varphi: N^{\prime} \rightarrow N$ surjective.

Case (3) : Restriction to fibers when the index $\left[N: \varphi\left(N^{\prime}\right)\right]$ is finite.

The maps $N^{\prime} \rightarrow \varphi\left(N^{\prime}\right) \hookrightarrow N$ induce a decomposition of $\tilde{\varphi}: X_{\Sigma^{\prime}} \stackrel{\widetilde{\varphi}_{1}}{\rightarrow} X_{\Sigma} \stackrel{\tau_{1}}{\rightarrow} X_{\Sigma}$, where the first $\Sigma$ is a fan in $\varphi\left(N^{\prime}\right)_{\mathbb{R}}$ while the second $\Sigma$ is in $N_{\mathbb{R}}$. The induced fibration $\widetilde{\varphi}_{1}: Y^{\prime} \rightarrow X_{\Sigma}$ is already discussed in Cases (1) and (2) above. Thus, it remains to understand the map $\widetilde{\iota}_{1}$, which is a branched covering of order $\left[N: \varphi\left(N^{\prime}\right)\right]$ and is a special case of our discussion in Sec. 2.1. The number of sheets over $O_{\sigma}$, $\sigma \in \Sigma$, is the same as $\operatorname{Ind}(\sigma)$, which must satisfy $\operatorname{Ind}(\sigma) \leq\left[N: \varphi\left(N^{\prime}\right)\right]$. The branched locus $\operatorname{Br}(\widetilde{\varphi})$ of $\tilde{\varphi}$ is a union of $V(\sigma)$ with $\operatorname{Ind}(\sigma)<\left[N: \varphi\left(N^{\prime}\right)\right]$. The fiber of $\widetilde{\varphi}: Y^{\prime} \rightarrow X_{\Sigma}$ over a point $p$ is then the disjoint union $\left.\cup_{p_{i} \in \tau_{\iota_{1}}{ }^{1}(p)} \widetilde{\varphi}_{1}\right|_{Y^{\prime}} ^{-1}\left(p_{i}\right)$. This concludes the discussion.

Case (4): Restriction to fibers when the index $\left[N: \varphi\left(N^{\prime}\right)\right]$ is infinite.

This is the remaining case after Cases (1), (2), and (3); and it can be reduced to the discussions in Cases (1), (2), and (3) by considering the maps of fans $\Sigma^{\prime} \stackrel{\varphi_{2}}{\rightarrow} \Sigma_{\varphi} \stackrel{\text { ‘ }}{\rightarrow} \Sigma$ induced by $\varphi: N^{\prime} \rightarrow N \cap \varphi\left(N_{\mathbb{R}}^{\prime}\right) \hookrightarrow N$. This gives a decomposition $\widetilde{\varphi}=\widetilde{\varphi}_{2} \circ \widetilde{\iota}_{2}$. The surjective map $\left.\widetilde{\varphi}_{2}\right|_{Y^{\prime}}$ is already discussed in Case (3). Since our goal is to understand the induced fibration of $Y^{\prime}$, we may replace $\widetilde{\varphi}$ by $\widetilde{\varphi}_{2}$. This concludes our discussion.

In the string literatures, e.g. [K-S1] and [K-S2], Cox homogeneous coordinates are used to understand the fibrations of toric Calabi-Yau hypersurfaces. For completeness of discussion, we recall in the following remark their essential points and fit them into the setting above.

Remark 3.2.4 [Cox homogeneous coordinates]. Assume that $\tilde{\varphi}: X_{\Sigma^{\prime}} \rightarrow X_{\Sigma}$ is a fibration. Let $\Sigma^{\prime}(1)=\left\{v_{1}^{\prime}, \cdots, v_{I^{\prime}}^{\prime}\right\}, \Sigma(1)=\left\{v_{1}, \cdots, v_{I}\right\}$, and $\left(z_{1}^{\prime}, \cdots, z_{I^{\prime}}^{\prime}\right)$ and $\left(z_{1}, \cdots, z_{I}\right)$ be the corresponding homogeneous coordinates for $X_{\Sigma^{\prime}}$ and $X_{\Sigma}$ respectively. Since $\varphi: \Sigma^{\prime}(1) \rightarrow \Sigma(1)$ is now surjective, $\varphi\left(v_{i}^{\prime}\right)=r_{i j} z_{j}$ for some $r_{i j} \in \mathbb{Z}_{\geq 0}$ and $\varphi$ determines a polynomial map from $\mathbb{C}^{I^{\prime}}$ to $\mathbb{C}^{I}$ defined by

$$
\left(z_{1}^{\prime}, \cdots, z_{I^{\prime}}^{\prime}\right) \longrightarrow\left(z_{1}, \cdots, z_{I}\right)=\left(\prod_{i} z_{i}^{\prime r_{i 1}}, \cdots, \prod_{i} z_{i}^{\prime r_{i I}}\right)
$$

This map is equivariant with respect to the group actions involved and indeed descends to the toric morphism $\tilde{\varphi}: X_{\Sigma^{\prime}} \rightarrow X_{\Sigma}$. Let $Y^{\prime}$ be a $\Sigma^{\prime}$-regular (cf. Sec. 4.1) hypersurface in $X_{\Sigma^{\prime}}$ in the linear system $\mathcal{O}_{X_{\Sigma^{\prime}}}\left(D^{\prime}\right)$, where $D^{\prime}=\sum_{i} a_{i} D_{v_{i}^{\prime}}$. Then, 
in terms of the given homogeneous coordinates, $Y^{\prime}$ is described by the zero locus of the polynomial

$$
z^{\prime D^{\prime}} \sum_{m^{\prime} \in \Delta^{\prime} \cap M^{\prime}} c_{m^{\prime}} \prod_{i=1}^{\left|\Sigma^{\prime}(1)\right|} z_{i}^{\prime m^{\prime}\left(v_{i}^{\prime}\right)}=\sum_{m^{\prime} \in \Delta^{\prime} \cap M^{\prime}} c_{m^{\prime}} \prod_{i=1}^{\left|\Sigma^{\prime}(1)\right|} z_{i}^{\prime m^{\prime}\left(v_{i}^{\prime}\right)+a_{i}},
$$

where $\Delta^{\prime}$ is the polytope in $M^{\prime}$ determined by $D^{\prime}$. To manifest the fibration $\tilde{\varphi}$, rewrite this polynomial as (cf. Equation (18) in [K-S2])

$$
\sum_{\overline{\varphi^{\dagger}}\left(m^{\prime}\right) \in \overline{\Delta^{\prime}}} c_{\overline{\varphi^{\dagger}}\left(m^{\prime}\right)} \prod_{v_{k}^{\prime} \in \varphi^{-1}(0)} z_{k}^{\prime\left\langle\overline{\varphi^{\dagger}}\left(m^{\prime}\right), v_{k}^{\prime}\right\rangle}
$$

with

$$
c_{\overline{\varphi^{\dagger}}\left(m^{\prime}\right)}=\sum_{m^{\prime} \in \overline{\varphi^{\dagger}}\left(m^{\prime}\right)} c_{m^{\prime}} \prod_{v_{k}^{\prime} \notin \varphi^{-1}(0)} z_{k}^{\prime m^{\prime}\left(v_{k}^{\prime}\right)+a_{k}} .
$$

Formally, this is an equation for the fiber with coefficients $c_{\varphi^{\dagger}}\left(m^{\prime}\right)$ regarded as polynomial of coordinates of the base $X_{\Sigma}$.

To realize this explicitly, pick a $\xi: N \rightarrow N^{\prime}$ as in our discussion of induced morphism $Y^{\prime} \subset X_{\Sigma^{\prime}} \rightarrow X_{\Sigma}$. The map $\xi$ determines a decomposition into three factors :

$$
\begin{aligned}
& \prod_{v_{k}^{\prime} \notin \varphi^{-1}(0)} z_{k}^{\prime m^{\prime}\left(v_{k}^{\prime}\right)+a_{k}} \\
& \quad=\prod_{v_{j} \in \Sigma(1)} z_{j}^{\left\langle\xi^{\dagger} m, v_{j}\right\rangle} \cdot \prod_{v_{k}^{\prime} \notin \varphi^{-1}(0)} z_{k}^{\prime\left\langle\varphi^{\dagger}\left(m^{\prime}\right), v_{k}^{\prime}-\xi \circ \varphi\left(v_{k}\right)\right\rangle} \cdot \prod_{v_{k}^{\prime} \notin \varphi^{-1}(0)} z_{k}^{\prime a_{k}} .
\end{aligned}
$$

Observe that the first factor is the polynomial in the homogeneous coordinates of $X_{\Sigma}$ associated to $\xi^{\dagger}(m) \in M$ and is the function of the orbit $O_{\{0\}}$ of the base that appear in the proof of Lemma 3.2.1, the last factor is an overall factor that is independent of $\overline{\varphi^{\dagger}}\left(m^{\prime}\right)$, and the middle factor is projected out in this correspondence. This gives a correspondence of our discussion and the ones in homogeneous coordinates for the fiber over $O_{\{0\}}$ as seen in physics literature. This concludes the remark.

Remark. 3.2.5. When $\tilde{\varphi}: Y^{\prime} \rightarrow X_{\Sigma}$ is a fibration, though in principle one can write down the equation of the discriminant locus of $\tilde{\varphi}$ but practically it remains very difficult to understand its details (cf. Example 4.2.1).

\section{Fibration of Calabi-Yau hypersurfaces via toric morphisms.}

The theme of this section is a computational scheme given and demonstrated in Sec. 4.2. Before getting into it, we give a couple of related remarks in Sec. 4.1. 


\subsection{General remarks.}

Remark 4.1.1 [Batyrev]. We recall some definitions and facts about toric hypersurfaces from $[\mathrm{Ba} 2]$ that will be needed in Sec. 4.2.

- Let $\Sigma^{\prime \prime}$ be a fan in $N_{\mathbb{R}}^{\prime \prime}$. Then the toric orbit decomposition of the ambient toric variety $X_{\Sigma^{\prime \prime}}$ induces a stratification of a hypersurface $Y^{\prime \prime}$ in $X_{\Sigma^{\prime \prime}}$ : $Y^{\prime \prime}=\cup_{\sigma^{\prime \prime} \in \Sigma^{\prime \prime}} Y_{\sigma^{\prime \prime}}^{\prime \prime} . Y^{\prime \prime}$ is called $\Sigma^{\prime \prime}$-regular if each stratum $Y_{\sigma^{\prime \prime}}^{\prime \prime}$ is either empty or a smooth subvariety of codimension 1 on $O_{\sigma^{\prime \prime}}$. In this case, this decomposition is a refinement of the stratification of the hypersurface by the analytic isomorphism classes of singularities. When $\Sigma^{\prime \prime}$ is the normal fan of a polytope $\Delta^{\prime}$, a $\Sigma^{\prime \prime}$-regular hypersurface is also called a $\Delta^{\prime}$-regular hypersurface.

- Fact $\left[\Delta^{\prime}\right.$-regular dense]. ([Proposition 3.1.3 in [Ba2].) The set of $\Delta^{\prime}$-regular hypersurfaces is a Zariski open subset in $\mathbb{P}\left(L\left(\Delta^{\prime}\right)\right)$, where $L\left(\Delta^{\prime}\right)$ is the space of Laurent polynomials with Newton polytope $\Delta^{\prime}$. For such hypersurfaces, the singularities are induced from the ambient toric variety.

- The local model for the transverse singularity along the stratum $Y_{\sigma^{\prime \prime}}^{\prime \prime}$ of the induced stratification is given by the affine toric variety $U_{\sigma^{\prime \prime}}, N_{\sigma^{\prime \prime}}^{\prime \prime}$ associated to the cone $\sigma^{\prime \prime}$ regarded as a fan in $N_{\sigma^{\prime \prime}}^{\prime \prime} \mathbb{R}$.

Lemma 4.1.2 [regularity]. Let $\Sigma^{\prime}$ corresponds to a maximal projective triangulation of the dual polytope $\Delta^{\prime *}$ of $\Delta^{\prime}$, then a $\Delta^{\prime}$-regular hypersurface in $X_{\Delta^{\prime}}$ becomes a $\Sigma^{\prime}$-regular hypersurface in $X_{\Sigma^{\prime}}$ after the associated maximal projective crepant partial (MPCP-)desingularization.

Proof. Let $\Sigma_{\Delta^{\prime}}$ be the normal fan of $\Delta^{\prime}$, which consists of cones over the faces of $\Delta^{\prime *}$, and consider the toric morphism $\zeta: X_{\Sigma^{\prime}} \rightarrow X_{\Delta^{\prime}}$ associated to the triangulation of $\Delta^{\prime *}$. Recall from Proposition 2.1.4 and its proof that the exceptional locus of $\zeta: X_{\Sigma^{\prime}} \rightarrow X_{\Delta^{\prime}}$ is a union of the orbit closures of $X_{\Sigma^{\prime}}$ associated to primitive cones in $\Sigma^{\prime}$ with respect to $\zeta$ and that, over an orbit $O_{\sigma^{\prime \prime}}$ of $X_{\Delta^{\prime}}, \zeta^{-1}\left(O_{\sigma^{\prime \prime}}\right)=O_{\sigma^{\prime \prime}} \times X\left(\sigma^{\prime \prime}\right)$ for some possibly reducible toric variety $X\left(\sigma^{\prime \prime}\right)$ and is the union of toric orbits $\cup_{\sigma^{\prime} \subset \operatorname{Int}\left(\sigma^{\prime \prime}\right)} O_{\sigma^{\prime}}$ in $X_{\Sigma^{\prime}}$. Consider now the restriction to hypersurfaces $\zeta: Y^{\prime} \rightarrow Y^{\prime \prime}$. For $\sigma^{\prime} \subset \operatorname{Int}\left(\sigma^{\prime \prime}\right), Y_{\sigma^{\prime}}^{\prime}=\zeta^{-1}\left(Y^{\prime \prime}\right) \cap O_{\sigma^{\prime}}=Y_{\sigma^{\prime \prime}}^{\prime \prime} \times O_{\sigma^{\prime}, \sigma^{\prime \prime}}$, where $O_{\sigma^{\prime}, \sigma^{\prime \prime}}$ is the toric orbit in $X\left(\sigma^{\prime \prime}\right)$ associated to $\sigma^{\prime}$. From this, $Y_{\sigma^{\prime}}^{\prime}$ must be empty if $Y_{\sigma^{\prime \prime}}^{\prime \prime}$ is empty or smooth of codimension 1 in $O_{\sigma^{\prime}}$ if $Y_{\sigma^{\prime \prime}}^{\prime \prime}$ is smooth of codimension 1 in $O_{\sigma^{\prime \prime}}$. This concludes the proof.

\subsection{The computational scheme and a detailed study of an example.}

The discussions in the previous subsections together provide us with a toric computational scheme to study the induced fibration of a toric Calabi-Yau hypersurface, 
as outlined below :

- The polytope $\Delta^{\prime}$ and the toric variety $X_{\Sigma^{\prime}}$ :

- The reflexive polytope $\Delta^{\prime}$.

- The lattice points in $\Delta^{\prime} \cap M^{\prime}$.

- The dual reflexive polytope $\Delta^{\prime *}$ in $N_{\mathbb{R}}^{\prime}$.

- The triangulation of $\Delta^{\prime *}$ that gives $\Sigma^{\prime}$.

- The singular locus of $X_{\Sigma^{\prime}}$.

- The line bundle $\mathcal{L}_{\Delta^{\prime}}$ and its restrictions:

- The restriction of $\mathcal{L}_{\Delta^{\prime}}$ to $V\left(\tau^{\prime}\right)$ for $\tau^{\prime}$ primitive in $\Sigma_{\sigma}^{\prime}$ for some $\sigma \in \Sigma$ :

* the polytope $\Delta_{\tau^{\prime}}^{\prime}$ in $\tau_{\mathbb{R}}^{\prime \perp}$, up to translation.

* the lattice points in $\Delta_{\tau^{\prime}}^{\prime} \cap \tau^{\prime \perp}$.

- The irreducible component $F_{\sigma}^{\tau^{\prime}}$ of a generic fiber of $\widetilde{\varphi}: V\left(\tau^{\prime}\right) \rightarrow V(\sigma)$.

- The restriction $\mathcal{L}_{F_{\sigma}^{\tau^{\prime}}}$ of $\mathcal{L}$ to $F_{\sigma}^{\tau^{\prime}}$ :

* the polytope $\overline{\Delta_{\tau^{\prime}}^{\prime}}$ in $\tau^{\prime \perp} / \varphi^{\dagger}\left(\sigma^{\perp}\right)$.

* The lattice points of the polytope $\overline{\Delta_{\tau^{\prime}}^{\prime}}$ in $\tau^{\perp \perp} / \varphi^{\dagger}\left(\sigma^{\perp}\right)$.

- The discriminant locus associated to $\overline{\Delta_{\tau^{\prime}}^{\prime}}$ in $\tau^{\prime \perp} / \varphi^{\dagger}\left(\sigma^{\perp}\right)$. (I.e. the discriminant locus of the family of sections of the line bundle $\mathcal{L}_{\overline{\Delta_{\tau^{\prime}}^{\prime}}}$ over $F_{\sigma}^{\tau^{\prime}}$.)

- The $\Sigma^{\prime}$-regular sections in $\mathcal{L}_{\Delta^{\prime}}$ and their restrictions :

- A $\Sigma^{\prime}$-regular section $s$ of $\mathcal{L}_{\Delta^{\prime}}$ and its zero-locus, the Calabi-Yau hypersurface $Y^{\prime}$.

- The singular loci of $Y^{\prime}$.

- The restriction of $s$ to $V\left(\tau^{\prime}\right)$ for $\tau^{\prime}$ primitive in $\Sigma_{\sigma}^{\prime}$ for some $\sigma \in \Sigma$.

- The restriction of $s$ to $F_{\sigma}^{\tau^{\prime}}$ :

* description of the irreducible components of a generic fiber of $\tilde{\varphi}: Y^{\prime} \rightarrow X_{\Sigma}$ over each of the toric orbits of $X_{\Sigma}$.

* restriction of a section of $\mathcal{L}_{\Delta^{\prime}}$ to $V\left(\tau^{\prime}\right)$ and then rewrite it in the fibred form with respect to a map $\xi_{\sigma}^{\tau^{\prime}}: N / N_{\sigma} \rightarrow N^{\prime} / N_{\tau^{\prime}}^{\prime}$.

- The induced fibration $Y^{\prime} \rightarrow X_{\Sigma}$ and the stratification of the discriminant locus of the fibration.

- Beyond :

- Note that before resolving the singularities of $Y^{\prime}$, all the fibers, generic and special alike, are described as hypersurfaces of the (perhaps reducible) fiber toric varieties.

- Fibers after crepant resolution of the singularities of $Y^{\prime}$. Note that now some of the fibers may not be realizable as hypersurfaces of fibers of $X_{\Sigma^{\prime}} \rightarrow X_{\Sigma}$.

- Monodromy of the fibration.

- Other details.

The sub-items in Item Beyond are not discussed in general in the current work. We will study some part of it in the specific example below. The purely toric items of this scheme should be programmable, (cf. Problem 5.1 in Sec. 5). 
We now apply this to the example of elliptic Calabi-Yau 4-folds in [B-C-dlO-G].

Example 4.2.1 [B-C-dlO-G]. (Continuing Example 2.2.2.) Let us now consider the induced fibration of a 4-dimensional Calabi-Yau hypersurface $Y^{\prime}$ in $X_{\Sigma^{\prime}}$ over $X_{\Sigma}$ from Example 2.2.2. All the notations follow from that example.

- The polytope $\Delta^{\prime}$ and the toric variety $X_{\Sigma^{\prime}}$ :

- The reflexive polytope $\Delta^{\prime}$ :

(1) $\Delta^{\prime}$ is the convex hull in $M_{\mathbb{R}}^{\prime}$ of the following set of 14 generating vertices in $M^{\prime}$ :

$$
\left\{\begin{array}{ll}
m_{1}^{\prime}=(-22,-14,4,1,1), & m_{2}^{\prime}=(-22,6,4,1,1), \\
m_{3}^{\prime}=(-10,-6,2,-1,1), & m_{4}^{\prime}=(-10,2,2,-1,1), \\
m_{5}^{\prime}=(-6,-6,0,1,1), & m_{6}^{\prime}=(0,0,0,-2,1), \\
m_{7}^{\prime}=(0,0,0,1,-1), & m_{8}^{\prime}=(2,-6,2,-1,1), \\
m_{9}^{\prime}=(2,2,2,-1,1), & m_{10}^{\prime}=(6,-14,4,1,1), \\
m_{11}^{\prime}=(6,-6,0,1,1), & m_{12}^{\prime}=(6,3,-3,1,1), \\
m_{13}^{\prime}=(6,6,-3,1,1), & m_{14}^{\prime}=(6,6,4,1,1)
\end{array}\right\} .
$$

(2) There are 9 codimension-1 faces, i.e. the maximal polytope of the boundary $\partial \Delta^{\prime}$ : (For brevity of notaion, only the indices $i$ of the generating $m_{i}^{\prime}$ are indicated. Their dual vertices in $N^{\prime}$ are labelled as subscripts.)

$$
\left\{\begin{array}{ll}
{[6,7,8,9,10,11,12,13,14]_{v_{1}^{\prime}},} & {[2,4,6,7,9,13,14]_{v_{2}^{\prime}},} \\
{[3,4,6,7,8,9,]_{c_{1}^{\prime}},} & {[1,2,3,4,7,8,9,10,14]_{c_{2}^{\prime}},} \\
{[1,2,5,7,10,11,12,13,14]_{v_{4}^{\prime}},} & {[1,2,3,4,5,6,8,9,10,11,12,13,14]_{v_{5}^{\prime}},} \\
{[1,3,5,6,7,8,10,11,]_{f^{\prime}},} & {[5,6,7,11,12]_{g^{\prime}},} \\
{[1,2,3,4,5,6,7,12,13]_{v_{6}^{\prime}}} &
\end{array}\right\} .
$$

Faces of higher codimensions are obtained by the intersection of subcollections of the above faces.

- The lattice points in $\Delta^{\prime} \cap M^{\prime}$ :

The cardinality $\left|\Delta^{\prime} \cap M^{\prime}\right|=3365$. Some sample lattice points in $\Delta^{\prime} \cap M^{\prime}$ are

$$
\begin{array}{lllll} 
& (-22,-14,4,1,1), & (-22,-13,4,1,1), & (-22,-12,4,1,1), & \ldots \\
\cdots, & (0,0,0,-1,1), & (0,0,0,0,0), & (0,0,0,0,1), & \ldots \\
\ldots, & (6,6,2,1,1), & (6,6,3,1,1), & (6,6,4,1,1) . &
\end{array}
$$

- The dual reflexive polytope $\Delta^{\prime *}$ in $N_{\mathbb{R}}^{\prime}$ :

(1) $\Delta^{\prime *}$ is the convex hull in $N_{\mathbb{R}}^{\prime}$ of the following set of 9 generating vertices in $N^{\prime}:\left\{v_{1}^{\prime}, v_{2}^{\prime}, c_{1}^{\prime}, c_{2}^{\prime}, v_{4}^{\prime}, v_{5}^{\prime}, f^{\prime}, g^{\prime}, v_{6}^{\prime}\right\}$. 
(2) There are 14 codimension-1 faces: (Their dual vertices in $M^{\prime}$ are labelled as subscripts.)

$$
\left\{\begin{array}{ll}
{\left[c_{2}^{\prime}, v_{4}^{\prime}, v_{5}^{\prime}, f^{\prime}, v_{6}^{\prime}\right]_{m_{1}^{\prime}},} & {\left[v_{2}^{\prime}, c_{2}^{\prime}, v_{4}^{\prime}, v_{5}^{\prime}, v_{6}^{\prime}\right]_{m_{2}^{\prime}},} \\
{\left[c_{1}^{\prime}, c_{2}^{\prime}, v_{5}^{\prime}, f^{\prime}, v_{6}^{\prime}\right]_{m_{3}^{\prime}},} & {\left[v_{2}^{\prime}, c_{1}^{\prime}, c_{2}^{\prime}, v_{5}^{\prime}, v_{6}^{\prime}\right]_{m_{4}^{\prime}},} \\
{\left[v_{4}^{\prime}, v_{5}^{\prime}, f^{\prime}, g^{\prime}, v_{6}^{\prime}\right]_{m_{5}^{\prime}},} & {\left[v_{1}^{\prime}, v_{2}^{\prime}, c_{1}^{\prime}, v_{5}^{\prime}, f^{\prime}, g^{\prime}, v_{6}^{\prime}\right]_{m_{6}^{\prime}},} \\
{\left[v_{1}^{\prime}, v_{2}^{\prime}, c_{1}^{\prime}, c_{2}^{\prime}, v_{4}^{\prime}, f^{\prime}, g^{\prime}, v_{6}^{\prime}\right]_{m_{7}^{\prime}},} & {\left[v_{1}^{\prime}, c_{1}^{\prime}, c_{2}^{\prime}, v_{5}^{\prime}, f^{\prime}\right]_{m_{8}^{\prime}},} \\
{\left[v_{1}^{\prime}, v_{2}^{\prime}, c_{1}^{\prime}, c_{2}^{\prime}, v_{5}^{\prime}\right]_{m_{9}^{\prime}},} & {\left[v_{1}^{\prime}, c_{2}^{\prime}, v_{4}^{\prime}, v_{5}^{\prime}, f^{\prime}\right]_{m_{10}^{\prime}},} \\
{\left[v_{1}^{\prime}, v_{4}^{\prime}, v_{5}^{\prime}, f^{\prime}, g^{\prime}\right]_{m_{11}^{\prime}},} & {\left[v_{1}^{\prime}, v_{4}^{\prime}, v_{5}^{\prime}, g^{\prime}, v_{6}^{\prime}\right]_{m_{12}^{\prime}},} \\
{\left[v_{1}^{\prime}, v_{2}^{\prime}, v_{4}^{\prime}, v_{5}^{\prime}, v_{6}^{\prime}\right]_{m_{13}^{\prime}},} & {\left[v_{1}^{\prime}, v_{2}^{\prime}, c_{2}^{\prime}, v_{4}^{\prime}, v_{5}^{\prime},\right]_{m_{14}^{\prime}}^{\prime}}
\end{array}\right\} .
$$

Faces of higher codimensions are obtained by the intersection of subcollections of the above faces.

- The triangulation of $\Delta^{\prime *}$ that gives $\Sigma^{\prime}$ :

(0) In general, triangulations of a polytope by lattice points can be generated, using the packages "PUNTOS", developed by Loera ([Lo]) and "PORTA", developed by Christof ([Ch1-2] and [Zi]). ${ }^{1}$ In this example, the triangulation we use is given in Example 2.2.2 in Sec. 2.2, following [B-C-dlO-V].

(1) The vertices $b^{\prime}, e_{1}^{\prime}, e_{2}^{\prime}$, and $e_{3}^{\prime}$ that lie in the boundary of $\Delta^{\prime *}$ are added to the vertex set to give a triangulation of $\Delta^{\prime *}$, the cones - with apex at the origin - over which form the fan $\Sigma^{\prime}$.

(2) The relation of $b^{\prime}, e_{1}^{\prime}, e_{2}^{\prime}$, and $e_{3}^{\prime}$ with the generating vertices of $\Delta^{\prime *}$ is given by $b^{\prime}=\left(v_{1}^{\prime}+4 c_{1}^{\prime}+v_{6}^{\prime}\right) / 6, e_{1}^{\prime}=\left(v_{1}^{\prime}+c_{1}^{\prime}+v_{6}^{\prime}\right) / 3$, $e_{2}^{\prime}=\left(v_{1}^{\prime}+v_{6}^{\prime}\right) / 2$, and $e_{3}^{\prime}=\left(v_{1}^{\prime}+2 v_{5}^{\prime}+v_{6}^{\prime}\right) / 4$.

- The singular locus of $X_{\Sigma^{\prime}}$ :

(1) The multiplicity mult $\left(\sigma^{\prime}\right)$ of $\sigma^{\prime} \in \Sigma^{\prime}$ is in the set $\{1,2,3\}$. Since 2 and 3 are primes and $\Sigma^{\prime}$ is a simplicial fan, if $\operatorname{mult}\left(\tau^{\prime}\right)>1$ and $\tau^{\prime} \prec \sigma^{\prime}$, then $\operatorname{mult}\left(\tau^{\prime}\right)=\operatorname{mult}\left(\sigma^{\prime}\right)$.

(2) Cones in $\Sigma^{\prime}$ whose multiplicity $>1$ but all of whose faces have multiplicity 1 are given by :

$$
\begin{array}{ll}
\text { multiplicity } 2: & v_{5}^{\prime} b^{\prime} ; \\
\text { multiplicity } 3: & v_{4}^{\prime} b^{\prime}, v_{4}^{\prime} e_{1}^{\prime} e_{2}^{\prime} .
\end{array}
$$

From the fan structure of $\Sigma^{\prime}$, the three orbit closurs $V\left(v_{5}^{\prime} b^{\prime}\right)$, $V\left(v_{4}^{\prime} b^{\prime}\right)$, and $V\left(v_{4}^{\prime} e_{1}^{\prime} e_{2}^{\prime}\right)$ are disjoint from each other. Their union forms the singular locus of $X_{\Sigma^{\prime}}$. Any other cone with multiplicity $>1$ contains exactly one of cones $v_{5}^{\prime} b^{\prime}, v_{4}^{\prime} b^{\prime}, v_{4}^{\prime} e_{1}^{\prime} e_{2}^{\prime}$ in its face and hence is contained in the corrresponding orbit closure of the latter. (FIGURE 4-2-1.)

\footnotetext{
${ }^{1}$ We would like to thank Shinobu Hosono for providing us with these codes and many helpful guidances of their use.
} 
(3) The toric geometry of the singular locus and the transverse singularity along it is given below :

(3.1) $V\left(v_{5}^{\prime} b^{\prime}\right):$ A toric 3-variety whose fan is given by $\Sigma$ (recall the fan of the base). The transverse singularity of $X_{\Sigma^{\prime}}$ along the open dense orbit of $V\left(v_{5}^{\prime} b^{\prime}\right)$ is given by the surface $A_{1}$-singularity.

(3.2) $V\left(v_{4}^{\prime} b^{\prime}\right):$ A toric 3-variety whose fan is also given by $\Sigma$. The transverse singularity of $X_{\Sigma^{\prime}}$ along the open dense orbit of $V\left(v_{5}^{\prime} b^{\prime}\right)$ is given by the surface $A_{2}$-singularity.

(3.3) $V\left(v_{4}^{\prime} e_{1}^{\prime} e_{2}^{\prime}\right)$ : A toric 2-variety whose fan is given by

$$
\{(1,0),(0,1),(-1,0),(0,-1)\} .
$$

This is $\mathbb{C P}^{1} \times \mathbb{C P}^{1}$. The transverse singularity of $X_{\Sigma^{\prime}}$ along the open dense orbit of $V\left(v_{4}^{\prime} e_{1}^{\prime} e_{2}^{\prime}\right)$ is given by the quotient $\mathbb{C}^{3} / \mathbb{Z}_{3}$, where $\mathbb{Z}_{3}$ acts on $\mathbb{C}^{3}$ by $\left(z_{1}, z_{2}, z_{3}\right) \mapsto$ $\left(\omega z_{1}, \omega z_{2}, \omega z_{3}\right), \omega^{3}=1$.

(3.4) Since $b^{\prime}, v_{4}^{\prime}, v_{5}^{\prime}$ are all mapped to zero under $\varphi, V\left(v_{5}^{\prime} b^{\prime}\right)$ and $V\left(v_{4}^{\prime} b^{\prime}\right)$ are indeed two sections of the fibration $\tilde{\varphi}$. They correspond to the two singular points of the generic fiber $\mathrm{W} \mathbb{C P}^{2}(1,2,3)$ - one $A_{1}$-singularity and the other $A_{2}$-singularity. Similarly, since the cone $v_{4}^{\prime} e_{1}^{\prime} e_{2}^{\prime}$ is mapped to $r_{1}, V\left(v_{4}^{\prime} e_{1}^{\prime} e_{2}^{\prime}\right)$ sits only over $V\left(r_{1}\right)$.

The structure of these orbit closures are obtained from the fact that these cones come from extensions of the 3-dimensional fan $\Sigma^{\prime \prime}$ in Example 2.2.2. The transverse singularities follow from $[\mathrm{Ba} 2]$ and $[\mathrm{Fu}]$ and the symmetry of the fans involved with respect to the lattice structure. Along their lower dimensional orbits, it is the product

$$
\text { (the above singularity) } \times \mathbb{C}^{\text {codimension of that orbit }} \text {. }
$$
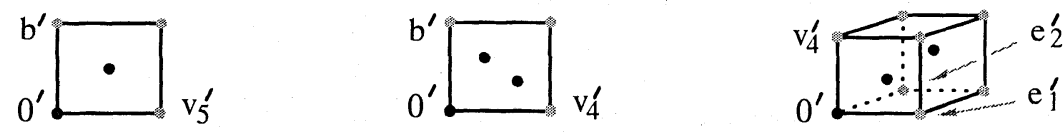

Figure 9: Figure 4-2-1. Multiplicity of $\left[v_{5}^{\prime}, b^{\prime}\right],\left[v_{4}^{\prime}, b^{\prime}\right]$, and $\left[v_{4}^{\prime}, e_{1}^{\prime}, e_{2}^{\prime}\right]$.

- The line bundle $\mathcal{L}_{\Delta^{\prime}}$ and its restrictions:

- $\mathcal{L}_{\Delta^{\prime}}$ has 3365 independent holomorphic sections. 
- The restriction of $\mathcal{L}_{\Delta^{\prime}}$ to $V\left(\tau^{\prime}\right)$ for $\tau^{\prime}$ primitive in $\Sigma_{\sigma}^{\prime}$ for some $\sigma \in \Sigma$ :

* the polytope $\Delta_{\tau^{\prime}}^{\prime}$ in $\tau_{\mathbb{R}}^{\prime \perp}$, up to translation: These are the convex hull of a subset of $\left\{m_{1}^{\prime}, \ldots, m_{14}^{\prime}\right\}$ determined by $\tau^{\prime}$. They are listed in the $3 k+2$ rows of TABLE $4-2-2$.

* the lattice points in $\Delta_{\tau^{\prime}}^{\prime} \cap \tau^{\prime \perp}$ : Their numbers are listed in the $3 k$ rows of TABLE 4-2-2.

- The irreducible component $F_{\sigma}^{\tau^{\prime}}$ of a generic fiber of $\tilde{\varphi}: V\left(\tau^{\prime}\right) \rightarrow V(\sigma)$ : These are given in Example 2.2.2, last culumn of TABLE 2-2-2. The intersection patterns for these are discussed in that example also, cf. FIGURE 2-2-3.

- The restriction $\mathcal{L}_{F_{\sigma}^{\tau^{\prime}}}$ of $\mathcal{L}$ to $F_{\sigma}^{\tau^{\prime}}$ :

* the polytope $\overline{\Delta_{\tau^{\prime}}^{\prime}}$ in $\tau^{\prime^{\perp}} / \varphi^{\dagger}\left(\sigma^{\perp}\right)$ : It turns out that, in this example, $\overline{\Delta_{\tau^{\prime}}^{\prime}}$ and hence $\mathcal{L}_{F_{\sigma}^{\tau^{\prime}}}$ depend only on the toric geometry of $F_{\sigma}^{\tau^{\prime}}$. They are given in Figure 4-2-3. From the $\overline{\Delta_{\tau^{\prime}}^{\prime}}$ worked out, one concludes that except for $F_{\sigma}^{\tau^{\prime}}$ that is isomorphic to the generic fiber $\mathrm{W} \mathbb{C P}^{2}(1,2,3)$, the restriction $\mathcal{L}_{F_{\sigma}^{\tau^{\prime}}}$ of $\mathcal{L}_{\Delta^{\prime}}$ to $F_{\sigma}^{\tau^{\prime}}$ is not the anticanonical line bundle of $F_{\sigma}^{\tau^{\prime}}$. One also observe that the various $\overline{\Delta_{\tau^{\prime}}^{\prime}}$ all come from some chopping of the polytope $\overline{\Delta_{\{0\}}^{\prime}}$ associated to the generic fiber $\mathrm{W} \mathbb{C P}^{2}(1,2,3)$.

* The lattice points of the polytope $\overline{\Delta_{\tau^{\prime}}^{\prime}}$ in $\tau^{\prime^{\perp}} / \varphi^{\dagger}\left(\sigma^{\perp}\right)$ : The polytopes are indicated in FIGURE 4-2-3. The numbers of lattice points they contain are listed below:

\begin{tabular}{|cccccc|}
\hline $\mathrm{W} \mathbb{C P}^{2}(1,2,3)$ & $X(4)$ & $\mathbb{C P}^{2}$ & $X(5)$ & $\mathrm{WCP}^{2}(1,1,3)$ & $\mathbb{F}_{2}$ \\
\hline 7 & 4 & 6 & 2 & 5 & 4 \\
\hline
\end{tabular}




\begin{tabular}{|c|c|c|c|}
\hline$O^{\prime}(54)$ & $v_{1}^{\prime}(27)$ & $v_{2}^{\prime}(24)$ & $c_{1}^{\prime}(16)$ \\
\hline all of $m_{i}^{\prime}$ & {$[6,7,8,9,10,11,12,13,14]$} & {$[2,4,6,7,9,13,14]$} & {$[3,4,6,7,8,9]$} \\
\hline 3365 & 227 & 262 & 154 \\
\hline$c_{2}^{\prime}(12)$ & $e_{1}^{\prime}(20)$ & $e_{2}^{\prime}(12)$ & $e_{3}^{\prime}(16)$ \\
\hline$[1,2,3,4,7,8,9,10,14]$ & {$[6,7]$} & {$[6,7,12,13]$} & {$[6,12,13]$} \\
\hline 1242 & 2 & 11 & 10 \\
\hline$f^{\prime}(16)$ & $g^{\prime}(20)$ & $v_{6}^{\prime} \quad(27)$ & $f^{\prime} c_{1}^{\prime}(8)$ \\
\hline$[1,3,5,6,7,8,10,11]$ & {$[5,6,7,11,12]$} & {$[1,2,3,4,5,6,7,12,13]$} & {$[3,6,7,8]$} \\
\hline 237 & 64 & 227 & 22 \\
\hline$f^{\prime} c_{2}^{\prime}(6)$ & $f^{\prime} g^{\prime}(6)$ & $g^{\prime} e_{1}^{\prime} \quad(10)$ & $g^{\prime} e_{2}^{\prime} \quad(6)$ \\
\hline$[3,7,8,10]$ & {$[5,6,7,11]$} & {$[6,7]$} & {$[6,7,12]$} \\
\hline 67 & 39 & 2 & 5 \\
\hline$g^{\prime} e_{3}^{\prime} \quad(8)$ & $v_{2}^{\prime} e_{1}^{\prime} \quad(10)$ & $v_{2}^{\prime} e_{2}^{\prime} \quad(6)$ & $v_{2}^{\prime} e_{3}^{\prime} \quad(8)$ \\
\hline$[6,12]$ & {$[6,7]$} & {$[6,7,13]$} & {$[6,13]$} \\
\hline 4 & 2 & 5 & 4 \\
\hline$v_{2}^{\prime} c_{1}^{\prime}(8)$ & $v_{2}^{\prime} c_{2}^{\prime} \quad(6)$ & $v_{1}^{\prime} v_{2}^{\prime}(12)$ & $v_{1}^{\prime} c_{1}^{\prime}(8)$ \\
\hline$[4,6,7,9]$ & {$[2,4,7,9,14]$} & {$[6,7,9,13,14]$} & {$[6,7,8,9]$} \\
\hline 22 & 86 & 26 & 16 \\
\hline$v_{1}^{\prime} c_{2}^{\prime} \quad(6)$ & $v_{1}^{\prime} e_{1}^{\prime} \quad(10)$ & $v_{1}^{\prime} e_{2}^{\prime} \quad(6)$ & $v_{1}^{\prime} e_{3}^{\prime}(8)$ \\
\hline$[7,8,9,10,14]$ & {$[6,7]$} & {$[6,7,12,13]$} & {$[6,12,13]$} \\
\hline 62 & 2 & 11 & 10 \\
\hline$v_{1}^{\prime} f^{\prime}(8)$ & $v_{1}^{\prime} g^{\prime} \quad(10)$ & $v_{6}^{\prime} v_{2}^{\prime} \quad(12)$ & $v_{6}^{\prime} c_{1}^{\prime} \quad(8)$ \\
\hline$[6,7,8,10,11]$ & {$[6,7,11,12]$} & {$[2,4,6,7,13]$} & {$[3,4,6,7]$} \\
\hline 19 & 14 & 26 & 16 \\
\hline $\begin{array}{ll}v_{6}^{\prime} c_{2}^{\prime} \quad(6) \\
\end{array}$ & $v_{6}^{\prime} e_{1}^{\prime} \quad(10)$ & $v_{6}^{\prime} e_{2}^{\prime}(6)$ & $\overline{v_{6}^{\prime} e_{3}^{\prime} \quad(8)}$ \\
\hline$[1,2,3,4,7]$ & {$[6,7]$} & {$[6,7,12,13]$} & {$[6,12,13]$} \\
\hline 62 & 2 & 11 & 10 \\
\hline$v_{6}^{\prime} f^{\prime}(8)$ & $v_{6}^{\prime} g^{\prime} \quad(10)$ & $v_{1}^{\prime} f^{\prime} c_{1}^{\prime}(4)$ & $\overline{v_{1}^{\prime} f^{\prime} c_{2}^{\prime} \quad(3)}$ \\
\hline$[1,3,5,6,7]$ & {$[5,6,7,12]$} & {$[6,7,8]$} & {$[7,8,10]$} \\
\hline 19 & 14 & 4 & 6 \\
\hline$v_{1}^{\prime} f^{\prime} g^{\prime} \quad(3)$ & $v_{1}^{\prime} g^{\prime} e_{1}^{\prime}(5)$ & $v_{1}^{\prime} g^{\prime} e_{2}^{\prime} \quad(3)$ & $\overline{v_{1}^{\prime} g^{\prime} e_{3}^{\prime} \quad(4)}$ \\
\hline$[6,7,11]$ & {$[6,7]$} & {$[6,7,12]$} & {$[6,12]$} \\
\hline 7 & 2 & 5 & 4 \\
\hline$v_{1}^{\prime} v_{2}^{\prime} e_{1}^{\prime} \quad(5)$ & $v_{1}^{\prime} v_{2}^{\prime} e_{2}^{\prime} \quad(3)$ & $\overline{v_{1}^{\prime} v_{2}^{\prime} e_{3}^{\prime} \quad(4)}$ & $\overline{v_{1}^{\prime} v_{2}^{\prime} c_{1}^{\prime}(4)}$ \\
\hline$[6,7]$ & {$[6,7,13]$} & {$[6,13]$} & {$[6,7,9]$} \\
\hline 2 & 5 & 4 & 4 \\
\hline$v_{1}^{\prime} v_{2}^{\prime} c_{2}^{\prime} \quad(3)$ & $v_{6}^{\prime} f^{\prime} c_{1}^{\prime} \quad(4)$ & $v_{6}^{\prime} f^{\prime} c_{2}^{\prime} \quad(3)$ & $\overline{v_{6}^{\prime} f^{\prime} g^{\prime} \quad(3)}$ \\
\hline$[7,9,14]$ & {$[3,6,7]$} & {$[1,3,7]$} & {$[5,6,7]$} \\
\hline 6 & 4 & 6 & 7 \\
\hline$v_{6}^{\prime} g^{\prime} e_{1}^{\prime} \quad(5)$ & $v_{6}^{\prime} g^{\prime} e_{2}^{\prime} \quad(3)$ & $v_{6}^{\prime} g^{\prime} e_{3}^{\prime} \quad(4)$ & $\overline{v_{6}^{\prime} v_{2}^{\prime} e_{1}^{\prime}(5)}$ \\
\hline$[6,7]$ & {$[6,7,12]$} & {$[6,12]$} & {$[6,7]$} \\
\hline 2 & 5 & 4 & 2 \\
\hline$v_{6}^{\prime} v_{2}^{\prime} e_{2}^{\prime} \quad(3)$ & $v_{6}^{\prime} v_{2}^{\prime} e_{3}^{\prime} \quad(4)$ & $v_{6}^{\prime} v_{2}^{\prime} c_{1}^{\prime}(4)$ & $\overline{v_{6}^{\prime} v_{2}^{\prime} c_{2}^{\prime} \quad(3)}$ \\
\hline$[6,7,13]$ & {$[6,13]$} & {$[4,6,7]$} & {$[2,4,7]$} \\
\hline 5 & 4 & 4 & 6 \\
\hline
\end{tabular}

TABLE 4-2-2. Primitive cones $\tau^{\prime}$ with respect to $\varphi$ are listed in the $3 k+1$ rows. The toric combinatorial complexity of $V\left(\tau^{\prime}\right)$ is indicated by the number of the maximal cones of its fan (in parenthesis). The generating vertices for the polytope $\Delta_{\tau^{\prime}}^{\prime}$ are indicated in the $3 k+2$ rows and the number of lattice points contained in it on the $3 k$ rows. 


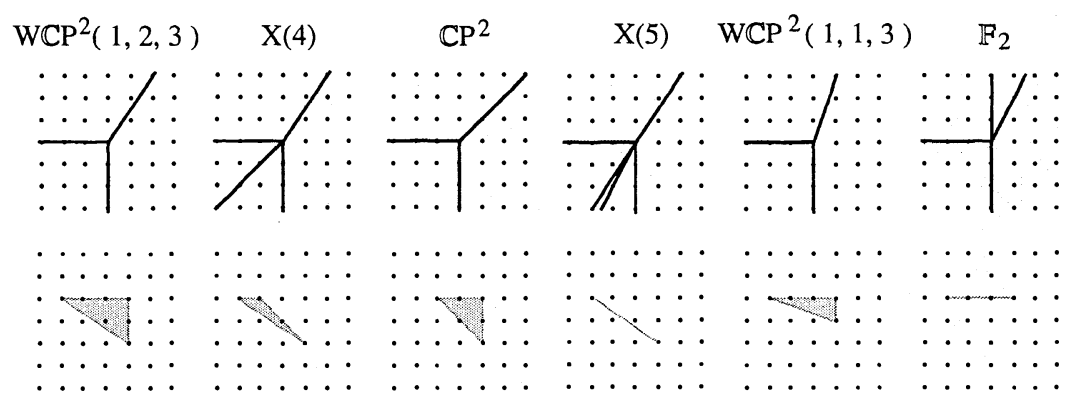

Figure 10: Figure 4-2-3. Tha fan for the six different types of irreducible components $F_{\sigma}^{\tau^{\prime}}$ of fibers of $\widetilde{\varphi}$ are listed in the first row. The restriction $\mathcal{L}_{F_{\sigma}^{\tau^{\prime}}}$ of $\mathcal{L}_{\Delta^{\prime}}$ to these fibers are described by the polytope $\overline{\Delta_{\tau^{\prime}}^{\prime}}$ indicated in the second row. Observe that the fan for $F_{\sigma}^{\tau^{\prime}}$ is a refinement of the normal fan of $\overline{\Delta_{\tau^{\prime}}^{\prime}}$ and that all the polytopes $\overline{\Delta_{\tau^{\prime}}^{\prime}}$ are some chopping of $\overline{\Delta_{\{0\}}^{\prime}}$.

- The discriminant associated to $\overline{\Delta_{\tau^{\prime}}^{\prime}}$ in $\tau^{\prime \perp} / \varphi^{\dagger}\left(\sigma^{\perp}\right)$ : They are related to the discriminant locus of the family of sections of the line bundle $\mathcal{L}_{\overline{\Delta_{\tau^{\prime}}^{\prime}}}$ over $F_{\sigma}^{\tau^{\prime}}$.

(1) For $\overline{\Delta_{\tau^{\prime}}^{\prime}}$ associated to $\mathrm{WCP}^{2}(1,2,3)$ : After the integral affine transformation on $M^{\prime}$ such that $(1,1) \rightarrow(0,0),(-2,1) \rightarrow(3,0),(1,-1) \rightarrow$ $(0,2)$, the local polynomial form of a section becomes

$$
f(x, y)=a_{00}+a_{10} x+a_{20} x^{2}+a_{30} x^{3}+a_{01} y+a_{11} x y+a_{02} y^{2} .
$$

Its discriminant is given by ([G-K-Z3] and [La])

$$
\begin{aligned}
\delta_{\overline{\Delta_{\tau^{\prime}}^{\prime}}} & =-27 \cdot 16 a_{00}^{2} a_{02}^{3} a_{30}^{2}-64 a_{00} a_{20}^{3} a_{02}^{3}-64 a_{10}^{3} a_{02}^{3} a_{30}-27 a_{01}^{4} a_{02} a_{30}^{2} \\
& +a_{00} a_{11}^{6}+16 a_{10}^{2} a_{20}^{2} a_{02}^{3}+16 a_{01}^{2} a_{20}^{3} a_{02}^{2}+a_{02} a_{10}^{2} a_{11}^{4}-a_{01} a_{10} a_{11}^{5} \\
& +a_{01}^{2} a_{11}^{4} a_{20}-a_{01}^{3} a_{11}^{3} a_{30}+32 \cdot 9 a_{00} a_{02}^{3} a_{10} a_{20} a_{30} \\
& +48 a_{00} a_{02}^{2} a_{11}^{2} a_{20}^{2}+8 \cdot 27 a_{00} a_{01}^{2} a_{02}^{2} a_{30}^{2},-72 a_{01}^{2} a_{02}^{2} a_{10} a_{20} a_{30} \\
& -72 a_{00} a_{02}^{2} a_{10} a_{11}^{2} a_{30}-16 a_{01} a_{02}^{2} a_{10} a_{11} a_{20}^{2}-8 a_{02}^{2} a_{10}^{2} a_{11}^{2} a_{20} \\
& +96 a_{01} a_{02}^{2} a_{10}^{2} a_{11} a_{30}-144 a_{00} a_{01} a_{02}^{2} a_{11} a_{20} a_{30}-12 a_{00} a_{02} a_{11}^{4} a_{20} \\
& +8 a_{01} a_{02} a_{10} a_{11}^{2} a_{20}-8 a_{01}^{2} a_{02} a_{11}^{2} a_{20}^{2}-30 a_{01}^{2} a_{02} a_{10} a_{11}^{2} a_{30} \\
& +36 a_{01}^{3} a_{02} a_{11} a_{20} a_{30}+36 a_{00} a_{01} a_{02} a_{11}^{3} a_{30}
\end{aligned}
$$

(2) For $\overline{\Delta_{\tau^{\prime}}^{\prime}}$ associated to $X(4)$ : After the integral affine transformation on $M^{\prime}$ such that $(-1,1) \rightarrow(0,0),(-2,1) \rightarrow(0,1),(-2,1) \rightarrow(2,0)$, the local polynomial form of a section becomes $f(x, y)=a_{00}+$ $a_{10} x+a_{20} x^{2}+a_{01} y$. Its discriminant is given by ([G-K-Z3]) $\delta_{\overline{\Delta_{\tau^{\prime}}^{\prime}}}=$ 1.

(3) For $\overline{\Delta_{\tau^{\prime}}^{\prime}}$ associated to $\mathbb{C P}^{2}$ : After the integral affine transformation on $M^{\prime}$ such that $(1,1) \rightarrow(0,0),(-1,1) \rightarrow(2,0),(1,-1) \rightarrow(0,2)$, 
the local polynomial form of a section becomes

$$
f(x, y)=a_{00}+a_{10} x+a_{20} x^{2}+a_{01} y+a_{11} x y+a_{02} y^{2} .
$$

Its discriminant is ([G-K-Z3])

$$
\delta_{\overline{\Delta_{\tau^{\prime}}^{\prime}}}=a_{20} a_{01}^{2}+a_{10}^{2} a_{02}+a_{11}^{2} a_{00}-a_{11} a_{10} a_{01}-4 a_{20} a_{02} a_{00} .
$$

(4) For $\overline{\Delta_{\tau^{\prime}}^{\prime}}$ associated to $X(5)$ : After an integral affine transformation on $M^{\prime}$ such that $(-2,1) \rightarrow(0,0),(1,-1) \rightarrow(1,0)$, the local polynomial form of a section becomes $f(x)=a_{0}+a_{1} x$. Its discriminant is given by ([G-K-Z3]) $\delta_{\overline{\Delta_{\tau^{\prime}}^{\prime}}}=a_{1}$.

(5) For $\overline{\Delta_{\tau^{\prime}}^{\prime}}$ associated to $\mathrm{WCP}^{2}(1,1,3)$ : After the integral affine transformation on $M^{\prime}$ such that $(1,1) \rightarrow(0,0),(-2,1) \rightarrow(3,0),(1,0) \rightarrow$ $(0,1)$, the local polynomial form of a section becomes

$$
f(x, y)=a_{00}+a_{10} x+a_{20} x^{2}+a_{30} x^{3}+a_{01} y .
$$

Its discriminant is given by $([\mathrm{G}-\mathrm{K}-\mathrm{Z} 3]) \delta_{\overline{\Delta_{\tau^{\prime}}^{\prime}}}=1$.

(6) For $\overline{\Delta_{\tau^{\prime}}^{\prime}}$ associated to $\mathbb{F}_{2}$ : After an integral affine transformation on $M^{\prime}$ such that $(-2,1) \rightarrow(0,0),(1,1) \rightarrow(3,0)$, the local polynomial form of a section becomes $f(x)=a_{0}+a_{1} x+a_{2} x^{2}+a_{3} x^{3}$. Its discriminant is given by ([G-K-Z3]) $\delta_{\overline{\Delta_{\tau^{\prime}}^{\prime}}}=27 a_{0}^{2} a_{3}^{2}+4 a_{0} a_{2}^{3}+$ $4 a_{1}^{3} a_{3}-a_{1}^{2} a_{2}^{2}-18 a_{0} a_{1} a_{2} a_{3}$.

- The $\Sigma^{\prime}$-regular sections in $\mathcal{L}_{\Delta^{\prime}}$ and their restrictions:

- A $\Sigma^{\prime}$-regular section s of $\mathcal{L}_{\Delta^{\prime}}$ and its zero-locus, the Calabi-Yau hypersurface $Y^{\prime}$ : The Hodge numbers of these Calabi-Yau hypersurfaces were computed in [B-C-dlO-G]: $h^{1,1}=8, h^{3,1}=2897, h^{2,1}=1$, and $h^{2,2}=11662$. The dimension of the polynomial complex moduli space $\mathcal{M}_{\text {poly }}$ is computed by the formula ([Ba2] and [C-K])

$$
\operatorname{dim}\left(\mathcal{M}_{\text {poly }}\right)=\left|\Delta^{\prime} \cap M^{\prime}\right|-6-\sum_{\Theta^{\prime}} l\left(\Theta^{\prime}\right)=2897,
$$

where $\Theta^{\prime}$ are the codimension-1 faces of $\Delta^{\prime}$ and $l\left(\Theta^{\prime}\right)$ is the number of lattice points in the relative interior of $\Theta^{\prime}$. Thus, the zero-locus of the 3365 -parameter family of sections of $\mathcal{L}_{\Delta^{\prime}}$ gives a 2897-parameter family of Calabi-Yau 4-varieties.

Remark. The triangulation of $\Delta^{\prime *}$ used so far missed 4 points in $\Delta^{\prime *} \cap N^{\prime}$. The latter come in when $X_{\Sigma^{\prime}}$ and hence $Y^{\prime}$ are desingularized. The above counting of moduli is more related to what comes after desingularization. 
- The singular loci of $Y^{\prime}$ : Under the assumption that $Y^{\prime}$ is $\Sigma^{\prime}$-regular, the singular locus $\operatorname{Sing}\left(Y^{\prime}\right)$ of $Y^{\prime}$ are induced from that of the ambient $X_{\Sigma^{\prime}}$. Thus, $\operatorname{Sing}\left(Y^{\prime}\right)=Y_{\left[v_{5}^{\prime} b^{\prime}\right]}^{\prime} \sqcup Y_{\left[v_{4}^{\prime} b^{\prime}\right]}^{\prime} \sqcup Y_{\left[v_{4}^{\prime} e_{1}^{\prime} e_{2}^{\prime}\right]}$. The first two components, if non-empty, have co-dimension 2 in $Y^{\prime}$ while the last component, if nonempty, has co-dimension 3.

- The restriction of $s$ to $V\left(\tau^{\prime}\right)$ for $\tau^{\prime}$ primitive in $\Sigma_{\sigma}^{\prime}$ for some $\sigma \in \Sigma$ : Suppose $s$ is given by

$$
s=\sum_{m^{\prime} \in \Delta^{\prime} \cap M^{\prime}} a_{m^{\prime}} \chi^{m^{\prime}}, \quad \text { (a total of } 3365 \text { summands). }
$$

Then

$$
\left.s\right|_{V\left(\tau^{\prime}\right)}=\sum_{m^{\prime} \in \Delta_{\tau^{\prime}}^{\prime} \cap M^{\prime}} a_{m^{\prime}} \chi^{m^{\prime}}, \quad \begin{aligned}
& \text { (only summands related to } \\
& \text { lattice points in } \Delta_{\tau^{\prime}}^{\prime} \text { remain). }
\end{aligned}
$$

The character $\chi^{m^{\prime}}, m^{\prime} \in \Delta_{\tau^{\prime}}^{\prime}$, is then rewritten as a character related to the lower dimensional torus associated to $V\left(\tau^{\prime}\right)$ by projecting it to $\tau^{\prime \perp}$. Let us give a concrete example.

Demonstration for $\left[v_{1}^{\prime}, e_{2}^{\prime}\right]$. Let $\tau^{\prime}=\left[v_{1}^{\prime}, e_{2}^{\prime}\right]$, then

$$
\Delta_{\left[v_{1}^{\prime}, e_{2}^{\prime}\right]}^{\prime}=\left[m_{6}^{\prime}, m_{7}^{\prime}, m_{12}^{\prime}, m_{13}^{\prime}\right]
$$

(cf. TABLE 4-2-2) and

$$
\Delta_{\left[v_{1}^{\prime}, e_{2}^{\prime}\right]}^{\prime} \cap M^{\prime}=\left\{\begin{array}{lll}
(0,0,0,-2,1), & (0,0,0,1,-1), & (2,1,-1,-1,1), \\
(2,2,-1,-1,1), & (4,2,-2,0,1), & (4,3,-2,0,1), \\
(4,4,-2,0,1), & (6,3,-3,1,1), & (6,4,-3,1,1), \\
(6,5,-3,1,1), & (6,6,-3,1,1) &
\end{array}\right\} .
$$

Fix a basis, e.g. $((2,0,-1,1,0),(0,1,0,0,0),(6,0,-3,0,2))$, for $\left[v_{1}^{\prime}, e_{2}^{\prime}\right]^{\perp} \cap$ $M^{\prime}$ and take, say $(0,0,0,-2,1)$, as the origin. Then

$$
\Delta_{\left[v_{1}^{\prime}, e_{2}^{\prime}\right]}^{\prime} \cap M^{\prime}=\left\{\begin{array}{llll}
(0,0,0), & (3,0,-1), & (1,1,0), & (1,2,0) \\
(2,2,0), & (2,3,0), & (2,4,0), & \\
(3,3,0), & (3,4,0), & (3,5,0), & (3,6,0)
\end{array}\right\} .
$$

and

$$
\begin{aligned}
& \left.s\right|_{V\left(\left[v_{1}^{\prime}, e_{2}^{\prime}\right]\right)}=a_{(0,0,0)}+a_{(3,0,-1)} t_{1}^{3} t_{3}^{-1}+a_{(1,1,0)} t_{1} t_{2}+a_{(1,2,0)} t_{1} t_{2}^{2} \\
& \quad+a_{(2,2,0)} t_{1}^{2} t_{2}^{2}+a_{(2,3,0)} t_{1}^{2} t_{2}^{3}+a_{(2,4,0)} t_{1}^{2} t_{2}^{4}+a_{(3,3,0)} t_{1}^{3} t_{2}^{3}+a_{(3,4,0)} t_{1}^{3} t_{2}^{4} \\
& \quad+a_{(3,5,0)} t_{1}^{3} t_{2}^{5}+a_{(3,6,0)} t_{1}^{3} t_{2}^{6} .
\end{aligned}
$$

To understand the meaning of this, fix a transverse dual basis, say $(0,0,0,1,0),(0,1,0,0,0),(0,0,-1,-1,-1))$, to the cone $\left[v_{1}^{\prime}, e_{2}^{\prime}\right]$ in $N^{\prime}$. The 6 maximal cones (cf. Table 4-2-2) in $\Sigma^{\prime}$ that contains $\left[v_{1}^{\prime}, e_{2}^{\prime}\right]$ in the face are

$$
v_{1}^{\prime} e_{3}^{\prime} e_{1}^{\prime} g^{\prime} e_{2}^{\prime}, \quad v_{1}^{\prime} e_{3}^{\prime} v_{4}^{\prime} g^{\prime} e_{2}^{\prime}, \quad v_{1}^{\prime} e_{1}^{\prime} v_{4}^{\prime} g^{\prime} e_{2}^{\prime}, \quad v_{1}^{\prime} e_{3}^{\prime} e_{1}^{\prime} v_{2}^{\prime} e_{2}^{\prime}, \quad v_{1}^{\prime} e_{3}^{\prime} v_{2}^{\prime} v_{4}^{\prime} e_{2}^{\prime}, \quad v_{1}^{\prime} e_{1}^{\prime} v_{2}^{\prime} v_{4}^{\prime} e_{2}^{\prime} \text {. }
$$

They project to the following six maximal cones in $\operatorname{Star}\left(\left[v_{1}^{\prime}, e_{2}^{\prime}\right]\right)$ :

$$
\bar{e}_{3}^{\prime} \bar{e}_{1}^{\prime} \bar{g}^{\prime}, \quad \bar{e}_{3}^{\prime} \bar{v}_{4}^{\prime} \bar{g}^{\prime}, \quad \bar{e}_{1}^{\prime} \bar{v}_{4}^{\prime} \bar{g}^{\prime}, \quad \bar{e}_{3}^{\prime} \bar{e}_{1}^{\prime} \bar{v}_{2}^{\prime}, \quad \bar{e}_{3}^{\prime} \bar{v}_{2}^{\prime} \bar{v}_{4}^{\prime}, \quad \bar{e}_{1}^{\prime} \bar{v}_{2}^{\prime} \bar{v}_{4}^{\prime}
$$


where

$\bar{e}_{1}^{\prime}=(1,0,3), \bar{e}_{3}^{\prime}=(0,0,-1), \bar{v}_{2}^{\prime}=(2,-1,6), \bar{v}_{4}^{\prime}=(-1,0,0), \bar{g}^{\prime}=(-1,1,-3)$.

In this way, $\left.\mathcal{L}\right|_{V\left(\left[v_{1}^{\prime}, e_{2}^{\prime}\right]\right)}$ is described directly by the 3 -dimensional polytope : the convex hull of $(0,0,0),(3,0,-1),(3,3,0)$, and $(3,6,0)$ and $\left.s\right|_{V\left(\left[v_{1}^{\prime} e_{2}^{\prime}\right]\right)}$ as given is a combination of characters on the 3 -dimensional torus $\left(\mathbb{C}^{\times}\right)^{3}$ associated to the cone: the origin of the rank 3 lattice in describing $\operatorname{Star}\left(\left[v_{1}^{\prime}, e_{2}^{\prime}\right]\right)$.

- The restriction of $s$ to $F_{\sigma}^{\tau^{\prime}}$ :

* description of the irreducible components of a generic fiber of $\widetilde{\varphi}$ : $Y^{\prime} \rightarrow X_{\Sigma}$ over each of the 33 toric orbits of $X_{\Sigma}$ :

(1) The essential feature depends only on the toric geometry of the fiber $F_{\sigma}$.

(2) For $F_{\sigma}=X(4) \cup \mathbb{C P}^{2}$ : With labels in Figure 2-2-3,

$$
\begin{array}{llrlrl}
\mathcal{L}_{c_{1}^{\prime}}=\mathcal{O}\left(D_{1}+D_{2}+D_{4}\right) & \text { and } & \left(D_{1}+D_{2}+D_{4}\right) \cdot D_{3}=2 & \text { on } X(4) ; \\
\mathcal{L}_{c_{2}^{\prime}}=\mathcal{O}\left(D_{5}+D_{6}\right) & \text { and } & & \left(D_{5}+D_{6}\right) \cdot D_{3}=2 & \text { on } \mathbb{C P}^{2} .
\end{array}
$$

These data restrict the generic degenerate fiber of $Y^{\prime}$ that lies in $X(4) \cup \mathbb{C P}^{2}$. (To be continued in Item: Beyond.)

(3) For $F_{\sigma}=X(5) \cup \mathrm{W} \mathbb{C P}^{2}(1,1,3) \cup \mathbb{F}_{2}$ : With labels in FiguRE 2-2-3,

$$
\begin{aligned}
& \mathcal{L}_{e_{1}^{\prime}}=\mathcal{O}\left(D_{1}+D_{2}-D_{3}+D_{5}\right) \text { and } \begin{array}{l}
\left(D_{1}+D_{2}-D_{3}+D_{5}\right) \cdot D_{3}=4 \\
\left(D_{1}+D_{2}-D_{3}+D_{5}\right) \cdot D_{4}=0 \\
\text { on } X(5) ;
\end{array} \\
& \mathcal{L}_{e_{2}^{\prime}}=\mathcal{O}\left(-D_{3}+D_{6}+D_{7}\right) \quad \text { and } \quad\left\{\begin{array}{l}
\left(-D_{3}+D_{6}+D_{7}\right) \cdot D_{3}=1 \\
\left(-D_{3}+D_{6}+D_{7}\right) \cdot D_{7}=3
\end{array}\right. \\
& \text { on } \mathrm{WCP}^{2}(1,1,3) \text {; } \\
& \mathcal{L}_{e_{3}^{\prime}}=\mathcal{O}\left(-D_{7}+D_{8}+D_{9}\right) \quad \text { and }\left\{\begin{array}{l}
\left(-D_{7}+D_{8}+D_{9}\right) \cdot D_{4}=0 \\
\left(-D_{7}+D_{8}+D_{9}\right) \cdot D_{7}=3 \\
\text { on } \mathbb{F}_{2} .
\end{array}\right.
\end{aligned}
$$

These data restrict the generic degenerate fiber of $Y^{\prime}$ that lies in $X(5) \cup \mathbb{W C P}^{2}(1,1,3) \cup \mathbb{F}_{2}$. (To be continued in Item: Beyond.)

* restriction of a section of $\mathcal{L}_{\Delta^{\prime}}$ to $V\left(\tau^{\prime}\right)$ and then rewrite it in the fibred form with respect to a map $\xi_{\sigma}^{\tau^{\prime}}: N / N_{\sigma} \rightarrow N^{\prime} / N_{\tau^{\prime}}^{\prime}$ : The details are already explained in the theory part. Let us give an explicit computation here for $\left[v_{1}^{\prime}, e_{2}^{\prime}\right]$ as an example.

Continuing Demonstration for $\left[v_{1}^{\prime}, e_{2}^{\prime}\right]$. Recall that $\varphi:\left[v_{1}^{\prime}, e_{2}^{\prime}\right] \mapsto$ $\left[d_{4}, r_{1}\right]$. Choose a compatible basis, say, $\left(d_{4}, r_{1},(0,1,0)\right)$ for $N$ and 
its dual basis for $M$. Extend the transverse basis to $\left[v_{1}^{\prime}, e_{2}^{\prime}\right]$ to the full basis, say $(0,0,0,1,0),(0,1,0,0,0),(0,0,-1,-1,-1),(0,0,2,2,3)$, $(1,0,0,-2,-3)$ for $N^{\prime}$ and take its dual basis for $M^{\prime}$. Then (treating vectors as column vectors)

$$
\varphi=\left[\begin{array}{rrrrr}
0 & 0 & 0 & 0 & -1 \\
0 & 0 & -1 & 2 & 0 \\
0 & 1 & 0 & 0 & 0
\end{array}\right]
$$

and the induced map on quotients $\bar{\varphi}_{d_{4} r_{1}}^{v_{1}^{\prime} e_{2}^{\prime}}: N^{\prime} / N_{v_{1}^{\prime} e_{2}^{\prime}}^{\prime} \rightarrow N / N_{d_{4} r_{1}}$ is given by $\bar{\varphi}_{d_{4} r_{1}}^{v_{1}^{\prime} e_{2}^{\prime}}=(0,1,0)$ and the dual $\bar{\varphi}_{d_{4} r_{1}}^{v_{1}^{\prime} e_{2}^{\prime}{ }^{\dagger}}:\left[d_{4}, r_{1}\right]^{\perp} \rightarrow v_{1}^{\prime} e_{2}^{\prime \perp}$ is given by $\bar{\varphi}_{d_{4} r_{1}}^{v_{1}^{\prime} e_{2}^{\prime}}=(0,1,0)^{t}$. From these, one obtains that

$$
\overline{\Delta_{\left[v_{1}^{\prime}, e_{2}^{\prime}\right]}^{\prime} \cap M^{\prime}}=\{(0,0),(1,0),(2,0),(3,0),(3,-1)\},
$$

as indicated in FiguRE 4-2-3, up to an integral affine transforma-


$\operatorname{Star}\left(\left[v_{1}^{\prime}, e_{2}^{\prime}\right]\right)$ that lie in $\bar{\varphi}_{d_{4} r_{1}}^{v_{1}^{\prime} e_{2}^{\prime}-1}(0)$ are given by $\bar{e}_{3}^{\prime} \bar{e}_{1}^{\prime}, \bar{e}_{3}^{\prime} \bar{v}_{4}^{\prime}, \bar{e}_{1}^{\prime} \bar{v}_{4}^{\prime}$, and their faces. Since $\bar{e}_{1}^{\prime} \rightarrow(1,3), \bar{e}_{3}^{\prime} \rightarrow(0,-1), \bar{v}_{4}^{\prime} \rightarrow(-1,0)$ in the 2-dimensional description, this fan describes $\operatorname{WCP}^{2}(1,1,3)$. Now choose $\xi_{d_{4} r_{1}}^{v_{1}^{\prime} e_{2}^{\prime}}: N / N_{d_{4} r_{1}} \rightarrow N^{\prime} / N_{v_{1}^{\prime} e_{2}^{\prime}}^{\prime}$ to be, say, $(0,1,0)^{t}$. This induces a rewriting of $\left.s\right|_{V\left(\left[v_{1}^{\prime}, e_{2}^{\prime}\right]\right)}$ as

$$
\begin{aligned}
\left.s\right|_{V\left(\left[v_{1}^{\prime}, e_{2}^{\prime}\right]\right)}=a_{(0,0,0)}+a_{(3,0,-1)} t_{1}^{3} t_{3}^{-1}+a_{(1,1,0)} t_{1} t_{2}+a_{(1,2,0)} t_{1} t_{2}^{2} \\
+a_{(2,2,0)} t_{1}^{2} t_{2}^{2},+a_{(2,3,0)} t_{1}^{2} t_{2}^{3}+a_{(2,4,0)} t_{1}^{2} t_{2}^{4}+a_{(3,3,0)} t_{1}^{3} t_{2}^{3} \\
\quad+a_{(3,4,0)} t_{1}^{3} t_{2}^{4}+a_{(3,5,0)} t_{1}^{3} t_{2}^{5}+a_{(3,6,0)} t_{1}^{3} t_{2}^{6} . \\
=a_{(0,0,0)}+\left(a_{(1,1,0)} t_{2}+a_{(1,2,0)} t_{2}^{2}\right) t_{1} \\
\quad+\left(a_{(2,2,0)} t_{2}^{2}+a_{(2,3,0)} t_{2}^{3}+a_{(2,4,0)} t_{2}^{4}\right) t_{1}^{2} \\
\quad+\left(a_{(3,3,0)} t_{2}^{3}+a_{(3,4,0)} t_{2}^{4}+a_{(3,5,0)} t_{2}^{5}+a_{(3,6,0)} t_{2}^{6}\right) t_{1}^{3} \\
\quad+a_{(3,0,-1)} t_{1}^{3} t_{3}^{-1} \\
=b_{(0,0)}\left(t_{2}\right)+b_{(1,0)}\left(t_{2}\right) t^{1}+b_{(2,0)}\left(t_{2}\right) t_{1}^{2}+b_{(3,0)}\left(t_{2}\right) t_{1}^{3} \\
\quad+b_{(3,-1)}\left(t_{2}\right) t_{1}^{3} t_{3}^{-1} .
\end{aligned}
$$

This is an expression of a section in $\left.\mathcal{L}\right|_{F_{d_{4} r_{1}}^{v_{1}^{\prime} e_{2}^{\prime}}}$ with coefficient functions $b_{(\cdot, \cdot)}\left(t_{2}\right)$ depending on the base parametrized by $t_{2} \in \mathbb{C}^{\times}$.

- The induced fibration $Y^{\prime} \rightarrow X_{\Sigma}$ and the stratification of the discriminant locus of the fibration: Once the section $s$ is $X_{\Sigma}$-orbit-wise written in the form of sections of $\mathcal{L}_{F_{\sigma}^{\tau^{\prime}}}$ with coefficients functions of $O_{\sigma}$, using the discriminant associated to $\overline{\Delta_{\tau^{\prime}}^{\prime}}$, one morally can study the stratification of the discriminant 
locus of the fibration. Unfortunately, for the current example, the number of parameters involved are so huge that it is above any tool known to us to analyze it.

- Beyond: Here we select only the issue of desingularization of this example for some discussions.

(1) Recall that

$\operatorname{Sing}\left(X_{\Sigma^{\prime}}\right)=V\left(\left[v_{5}^{\prime} b^{\prime}\right]\right) \sqcup V\left(\left[v_{4}^{\prime} b^{\prime}\right]\right) \sqcup V\left(\left[v_{4}^{\prime} e_{1}^{\prime} e_{2}^{\prime}\right]\right) \simeq X_{\Sigma} \sqcup X_{\Sigma} \sqcup \mathbb{C P}^{1} \times \mathbb{C P}^{1}$.

To desingularize $X_{\Sigma^{\prime}}$, one adds

$$
\begin{aligned}
& b_{3}^{\prime}=\left(v_{5}^{\prime}+b^{\prime}\right) / 2=(0,0,0,1,1) \\
& b_{1}^{\prime}=\left(v_{4}^{\prime}+2 b^{\prime}\right) / 3=(0,0,0,1,2), \quad b_{2}^{\prime}=\left(2 v_{4}^{\prime}+b^{\prime}\right) / 3=(0,0,0,0,1) \\
& e_{4}^{\prime}=\left(v_{4}^{\prime}+e_{1}^{\prime}+e_{2}^{\prime}\right) / 3=(0,0,1,1,2)
\end{aligned}
$$

to $\Sigma^{\prime}(1)$. Since these lattice points lie in $\Delta^{\prime *}$, they determine a unique refined triangulation of $\Delta^{\prime *}$, which in turn determines a refinement $\widetilde{\Sigma^{\prime}}$ of $\Sigma^{\prime}$. The following rules tell us how a simplicial cone in $\Sigma^{\prime}$ splits to simplicial cones in $\widetilde{\Sigma^{\prime}}$ :

$$
\begin{aligned}
& {\left[\cdots, v_{5}^{\prime}, b^{\prime}\right] \longrightarrow\left[\cdots, v_{5}^{\prime}, b_{3}^{\prime}\right]+\left[\cdots, b_{3}^{\prime}, b^{\prime}\right]} \\
& {\left[\cdots, v_{4}^{\prime}, b^{\prime}\right] \longrightarrow\left[\cdots, v_{4}^{\prime}, b_{2}^{\prime}\right]+\left[\cdots, b_{2}^{\prime}, b_{1}^{\prime}\right]+\left[\cdots, b_{1}^{\prime}, b^{\prime}\right]} \\
& {\left[\cdots, v_{4}^{\prime}, e_{1}^{\prime}, e_{2}^{\prime}\right] \longrightarrow\left[\cdots, e_{1}^{\prime}, e_{2}^{\prime}, e_{4}^{\prime}\right]+\left[\cdots, v_{4}^{\prime}, e_{2}^{\prime}, e_{4}^{\prime}\right]} \\
& +\left[\cdots, v_{4}^{\prime}, e_{1}^{\prime}, e_{4}^{\prime}\right]
\end{aligned}
$$

All other types of cones remain the same. Denote $\widetilde{X_{\Sigma^{\prime}}}$ the toric variety associated to the refined fan. One can check that all the new maximal cones have multiplicity 1 , thus $\widetilde{X_{\Sigma^{\prime}}}$ is a smooth 5fold. Since $Y^{\prime}$ is $\Sigma^{\prime}$-regular, its proper transform $\widetilde{Y^{\prime}}$ in $\widetilde{X_{\Sigma^{\prime}}}$ is also smooth.

(2) Since $\varphi: b_{3}^{\prime}, b_{1}^{\prime}, b_{2}^{\prime} \rightarrow 0, e_{4}^{\prime} \rightarrow r_{1}$, the set of primitive cones over $\left[\cdots, r_{1}\right]$ with respect to $\varphi$ now becomes

$$
\left\{\left[\cdots^{\prime}, e_{1}^{\prime}\right],\left[\cdots^{\prime}, e_{2}^{\prime}\right],\left[\cdots^{\prime}, e_{3}^{\prime}\right],\left[\cdots^{\prime}, e_{4}^{\prime}\right]\right\}
$$

The primitive cones over all other types of cones in $\Sigma$ remain the same.

(3) Following the same argument in earlier parts of the example, one has the following proper transform/resolution of components of fibers 
of $\tilde{\varphi}$ :

$$
\begin{aligned}
& \mathrm{W} \mathbb{C P}^{2}(1,2,3) \longleftarrow \widetilde{\mathrm{WCP}(1,2,3)}=X_{\{(1,1),(2,3),(1,2),(0,1),(-1,0),(0,-1)\}} \\
& \simeq \mathbb{C P}^{2} \sharp 3 \overline{\mathbb{C P}^{2}} \\
& X(4) \longleftarrow \widetilde{X(4)}=X_{\{(1,1),(2,3),(1,2),(0,1),(-1,0),(-1,-1),(0,-1)\}} \\
& \simeq \mathbb{C P}^{2} \sharp 4 \overline{\mathbb{C P}^{2}} \\
& \mathbb{C P}^{2} \longleftarrow \mathbb{C P}^{2} \\
& X(5) \longleftarrow \widetilde{X(5)}=X_{\{(1,1),(2,3),(1,2),(0,1),(-1,0),(-1,-1),(-2,-3),(-1,-2),(0,-1)\}} \\
& \simeq \mathbb{C P}^{2} \sharp 6 \overline{\mathbb{C P}^{2}} \\
& \left.\mathrm{~W} \mathbb{C P}^{2}(1,1,3) \longleftarrow \quad \widetilde{W} \widetilde{P^{2}(1,1}, 3\right)=X_{\{(1,3),(0,1),(-1,0),(0,-1)\}} \\
& \simeq \mathbb{F}_{3} \\
& \mathbb{F}_{2} \quad \longleftarrow \mathbb{F}_{2} \\
& \longleftarrow \mathbb{C P}^{2}
\end{aligned}
$$

The intersection relations of these components are induced from the original components and are indicated in FIgURE 4-2-4.

(4) The restriction of the pullback line bundle on $\widetilde{X_{\Sigma^{\prime}}}$, still denoted by $\mathcal{L}$, to these fibers are still described by the same polytopes, except for the new fiber component $\mathbb{C P}^{2}$ from the relative stars $\operatorname{Star}_{\left[\cdots, r_{1}\right]}\left[\cdots^{\prime}, e_{4}^{\prime}\right]$, the restriction of $\mathcal{L}$ to which is trivial. Note that none of the restriction is the anticanonical bundle except for the generic fiber $\widehat{\mathrm{W}} \widehat{\mathrm{P}^{2}(1,2,3)}$.

(4.1) For $F_{\sigma}=\widetilde{X(4)} \cup \mathbb{C P}^{2}$ : With labels in Figure 4-2-4,

$$
\begin{aligned}
& \mathcal{L}_{c_{1}^{\prime}}=\mathcal{O}\left(D_{1}+D_{2}+D_{4}+E_{1}+E_{2}+E_{3}\right) \\
& \quad \text { and }\left(D_{1}+D_{2}+D_{4}+E_{1}+E_{2}+E_{3}\right) \cdot D_{3}=2 \text { on } \widetilde{X(4)} ; \\
& \mathcal{L}_{c_{2}^{\prime}}=\mathcal{O}\left(D_{5}+D_{6}\right) \\
& \quad \text { and }\left(D_{5}+D_{6}\right) \cdot D_{3}=2 \text { on } \mathbb{C P}^{2} .
\end{aligned}
$$

(4.2). For $\left.F_{\sigma}=\widetilde{X(5)} \cup \mathrm{W} \widetilde{\mathbb{C P}^{2}(1,1}, 3\right) \cup \mathbb{F}_{2} \cup \mathbb{C P}^{2}$ : With labels in FIGURE 4-2-4,

$$
\begin{aligned}
& \mathcal{L}_{e_{1}^{\prime}}=\mathcal{O}\left(D_{1}+D_{2}-D_{3}+D_{5}+E_{1}+E_{2}+E_{3}\right) \\
& \text { and }\left\{\begin{array}{l}
\left(D_{1}+D_{2}-D_{3}+D_{5}+E_{1}+E_{2}+E_{3}\right) \cdot D_{3}=1 \\
\left(D_{1}+D_{2}-D_{3}+D_{5}+E_{1}+E_{2}+E_{3}\right) \cdot D_{4}=0
\end{array} \quad \text { on } \widetilde{X(5)} ;\right. \\
& \mathcal{L}_{e_{2}^{\prime}}=\mathcal{O}\left(-D_{3}+D_{6}+D_{7}\right) \\
& \text { and }\left\{\begin{array}{l}
\left(-D_{3}+D_{6}+D_{7}\right) \cdot D_{3}=1 \\
\left(-D_{3}+D_{6}+D_{7}\right) \cdot D_{7}=3
\end{array} \text { on } \widetilde{\mathrm{CP}^{2}(1,1,3) ;}\right. \\
& \mathcal{L}_{e_{3}^{\prime}}=\mathcal{O}\left(-D_{7}+D_{8}+D_{9}\right) \\
& \text { and }\left\{\begin{array}{l}
\left(-D_{7}+D_{8}+D_{9}\right) \cdot D_{4}=0 \\
\left(-D_{7}+D_{8}+D_{9}\right) \cdot D_{7}=3
\end{array} \quad \text { on } \mathbb{F}_{2} ;\right. \\
& \mathcal{L}_{e_{4}^{\prime}}=\mathcal{O} \text { on } \mathbb{C P}^{2} .
\end{aligned}
$$

(5) Recall the adjunction formula $g=1+\frac{1}{2}\left(K_{S}+C\right) \cdot C$ that computes the genus $g$ of a smooth embedded curve $C$ in the divisor class $[C]$ 
of a smooth surface $S$ via the intersection number of the canonical divisor $K_{S}$ of $S$ and the self-intersection number of $C$. One can check that all the smooth curves in $F_{\sigma}^{\widetilde{\tau^{\prime}}}$ cut out by sections of $\mathcal{L}_{F_{\sigma}} \widetilde{\tau^{\prime}}$, where $\widetilde{\tau^{\prime}}$ is any primitive cone in $\widetilde{\Sigma^{\prime}}$, are rational, as they should be. Since each type of fibers appear over a codimension- 1 stratum of the discriminant locus of the fibration $\widetilde{Y^{\prime}} \rightarrow X_{\Sigma}$, from the intersection numbers computed in Sub-item (4) above and the Kodaira's table of singular elliptic fibers of a 1-parameter family elliptic fibration, one concludes that: For the elliptic fibration $\widetilde{Y^{\prime}} \rightarrow X_{\Sigma}$ of Calabi-Yau 4-fold induced from the toric morphism $X_{\widetilde{\Sigma^{\prime}}} \rightarrow X_{\Sigma}$, the generic degenerate fiber associated to the $\widetilde{X(4)} \cup \mathbb{C P}^{2}$ toric fiber has two irreducible components and is of type III (or $\widetilde{A_{1}}$ ) in Kodaira's table while the generic degenerate fiber associated to the

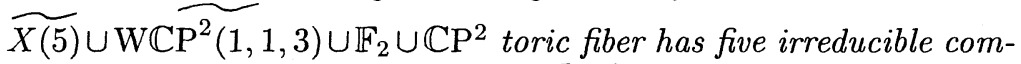
ponents (one in $\widetilde{X(5)}$, one in $\mathrm{W} \widetilde{\mathrm{CP}^{2}(1,1,3)}$ and three in $\mathbb{F}_{2}$ ) and is of type $I_{0}^{*}$ (or $\widetilde{D_{4}}$ ).
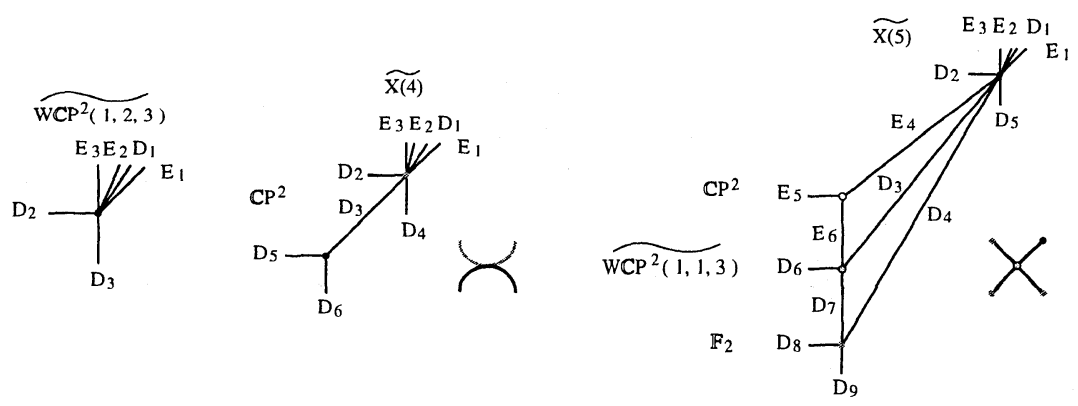

Figure 11: Figure 4-2-4. The toric divisors associated to 1-cones in the toric fiber of $\widetilde{X_{\Sigma^{\prime}}} \rightarrow X_{\Sigma}$ and the Kodaira labels for the generic fiber and the generic degenerate fibers of the elliptic Calabi-Yau hypersurface $Y^{\prime}$ after toric resolution of the singularities.

This concludes our long discussions of the example.

\section{Remarks on further study.}

At the end, we list five problems from our list for further pursuit. 
Problem 5.1 [link to Kreuzer-Skarke]. Elaboration of the work of Kreuzer and Skarke ([K-S1] and [K-S2]), which classifies higher dimensional reflexive polytopes that admit fibration, with the computational scheme presented in this paper. This would give further information of fibrations in their list.

Problem 5.2 [fibred Calabi-Yau variety]. The above huge number of concrete examples may provide us a hint for deeper understanding of general fibred CalabiYau varieties - toric and non-toric alike -. These examples may also shed some light on the generalization of Kodaira's table of elliptic fibrations to higher dimensional families.

Problem 5.3 [complete intersection]. Extension of the current work to higher rank equivariant vector bundles over a toric variety to take care of fibred Calabi-Yau varieties from complete intersections of hypersurfaces in a toric variety and a toric morphism. Realization of this will complete the first step toward understanding fibred Calabi-Yau variety in the toric category.

Problem 5.4 [effect on quantum cohomology]. In view of the goal that understanding a fibration is meant to understanding the original space in terms of lower dimensional ones and the data how they pile together, one would like to work out a generalized quantum Künneth formula for the quantum cohomology of fibred toric variety (cf. [Bal] and [K-M]) and also for the hypersurfaces therein. This problem currently looks extremely difficult.

Problem 5.5 [Het/F-theory duality]. Calabi-Yau hierarchy from toric morphisms: a purely toric realization of the Het/F-theory duality. This will be the analogue of the work of Batyrev on mirror symmetry of Calabi-Yau manifolds of the same dimensions. This problem is a final dream of all the participants of the heterotic-string/F-theiry duality in the toric category.

With these problems in mind for further investigations, let us conclude this paper and turn to another new pursuit. 


\section{References}

[A-G-M1] P.S. Aspinwall, B. Greene, and D.R. Morrison, Multiple mirror manifolds and topology change in string theory, Phys. Lett. B303 (1993), pp. 249 - 259.

[A-G-M2] — The monomial-divisor mirror map, Intern. Math. Res. Notices, (1993), no. 12, pp. 319 - 337.

[A-K-M] P.S. Aspinwall, S. Katz, and D.R. Morrison, Lie groups, Calabi-Yau threefolds, and F-theory, Adv. Theo. Math. Phys. 4 (2000), pp. 95 - 126.

[A-K-M-S] A.C. Avram, M. Kreuzer, M. Mandelberg, and H. Skarke, Searching for K3 fibrations, Nucl. Phys. B494 (1997), pp. 567 - 589.

[A-M] P.S. Aspinwall and D.R. Morrison, Point-like instantons on K3 orbifolds, Nucl. Phys. B503 (1997), pp. 533 - 564.

[Ba1] - Quantum cohomology rings of toric manifolds, Journées de Géométrie algébrique d'Orsay - Juillet 1992, Astérisque 218 (1993), pp. 9 - 34.

[Ba2] - Dual polyhedra and mirror symmetry for Calabi-Yau hypersurfaces in toric varieties, J. Alg. Geom. 3 (1994), pp. 493 - 535.

[Bo] L.A. Borisov, Towards the mirror symmetry for Calabi-Yau intersections in Gorenstein toric Fano varieties, alg-geom/9310001.

[B-B] V.V. Batyrev and L.A. Borisov, On Calabi-Yau complete intersections in toric varieties, in Higher-dimensional complex varieties, Trento 1994, M. Andreatta and T. Peternell eds., pp. 39 - 65, Walter de Gruyter, 1996.

[BB-S] A. Białynicki-Birula and A.J. Sommese, Quotients by $\mathbb{C}^{*}$ and $S L(2, \mathbb{C})$ actions, Trans. Amer. Math. Soc. 279 (1983), pp. 773 - 800.

[B-C-dlO-G] V. Braun, P. Candelas, X. de la Ossa, and A. Grassi, Toric Calabi-Yau fourfolds, duality between $N=1$ theories, and divisors that contribute to the superpotential, hep-th/0001208.

[B-D] V.V. Batyrev and D.I. Dais, Strong MaKay correspondence, string-theoretic Hodge numbers and mirror symmetry, Topology 35 (1996), pp. 901 - 929.

[B-I-K-M-S-V] M. Bershadsky, K. Intriligator, S. Kachru, D.R. Morrison, V. Sadov, and C. Vafa, Geometric singularities and enhanced gauge symmetries, Nucl. Phys. B481 (1996), pp. 215 - 252.

[B-J-P-S] M. Bershadsky, A. Johansen, T. Pantev, and V. Sadov, On four-dimensional compactifications of F-theory, Nucl. Phys. B505 (1997), pp. 165 - 201.

[B-M] P. Berglund and P. Mayr, Heterotic string/F-theory duality from mirror symmetry, Adv. Theo. Math. Phys. 2 (1998), pp. 1307 - 1372.

[B-P-VV] W. Barth, C. Peters, abd A. Van de Ven, Compact complex surfaces, Springer-Verlag, 1984.

[B-S] L.J. Billera and B. Sturmfels, Fiber polytopes, Ann. Math. 135 (1992), pp. 527 - 549.

[Ch1] T. Christof, Ein Verfahren zur Transformation zwischen Polyederdarstellungen, Thesis, Universität Augsburg, 1991.

[Ch2] - PORTA - A polyhedron representation transformation algorithm, written by T. Christof; revised by A. Loebel and M. Stoer (ZIB Berlin); available from the ZIB electronic library ELIB via elib@zib-berlin.de or by anolomous ftp from elib.zib-berlin.de directory "/pub/mathprog".

[Co] D.A. Cox, The homogeneous coordinate ring of a toric variety, J. Alg. Geom. 4 (1995), pp. $17-50$.

[C-F] P. Candelas and A. Font, Duality between the web of heterotic and type II vacua, Nucl. Phys. B511 (1998), pp. 295 - 325.

[C-K] D.A. Cox and S. Katz, Mirror symmetry and algebraic geometry, Math. Surv. Mono. 68, AMer. Math. Soc. 1999.

[C-P-R] P. Candelas, E. Perevalov, and G. Rajesh, Toric geometry and enhanced gauge symmetry of F-theory/heterotic vacua, Nucl. Phys. B507 (1997), pp. 445 - 474. 
[Da] V.I. Danilov, The geometry of toric varieties, Russian Math. Surveys 33 (1978), pp. $97-154$.

[Do] I. Dolgachev, Introduction to invariant theory, course given at the Department of Mathematica, Harvard University, fall 1999; lecture notes obtainable from the web site: 'www.math.lsa.umich.edu/ idolga/lecturenotes.html'.

[D-H] I.V. Dolgachev and Y. Hu, Variation of geometric invariant theory quotients, with an appendix by Nicolas Ressayre, I.H.E.S. Publ. Math. 87 (1998), pp. 5 - 56.

[D-R] D.-E. Diaconescu and G. Rajesh, Geometrical aspects of fivebranes in heterotic/Ftheory duality in four dimensions, J. High Energy Phys. (1999), hep-th/9903104.

[Ei] D. Eisenbud, Commutative algebra with a view toward algebraic geometry, GTM 150, Springer-Verlag 1995.

[Ew] G. Ewald, Combinatorial convexity and algebraic geometry, GTM 168, SpringerVerlag, 1996.

[E-H] D. Eisenbud and J. Harris, The geometry of schemes, GTM 197, Springer-Verlag, 2000.

[Fu] W. Fulton, Introduction to toric varieties, Ann. Math. Study 131, Princeton Univ. Press, 1993.

[F-M-W] R. Friedman, J. Morgan, and E. Witten, Vector bundles and F theory, Commun. Math. Phys. 187 (1997), pp. 679 - 743.

[Gr] B.R. Greene, String theory on Calabi-Yau manifolds, in Fields, strings, and duality, TASI 1996, C. Efthimiou and B.R. Greene eds., pp. 543 - 726, World Scientific, 1998.

[G-K-Z1] I.M. Gelfand, M.M. Kapranov, and A.V. Zelevinsky, Equations of hypergeometric type and toric varieties, Funct. Anal. Appl. 23 (1989), pp. 94 - 106.

[G-K-Z2] — Discriminants of polynomials in several variables and triangulations of Newton polytopes, Algebra i Analiz 2 no. 3 (1990), pp. 1-62.

[G-K-Z3] —, Discriminants, resultants, and multidimensional determinants, Birkhäuser, 1994.

[G-M-P] B.R. Greene, D.R. Morrison, and M.R. Plesser, Mirror manifolds in higher dimensions, Commun. Math. Phys. 173 (1995), pp. 559 - 598.

[G-MP] M. Goresky and R. MacPherson, On the topology of algebraic torus actions, in Algebraic groups, Utrecht 1986, A.M. Cohen, W.H. Hesselink, W.L.J. Van der Kallen, J.R. Strooker eds., pp. 73 - 90, Lect. Notes Math. 1271, Springer-Verlag, 1987.

[Ha] R. Hartshorne, Algebraic geometry, GTM 52, Springer-Verlag, 1977.

[Hu1] Y. Hu, The geometry and topology of quotient varieties of torus actions, Duke Math. J. 68 (1992), pp. 151 - 184.

[Hu2] — Combinatorics and quotients of toric varieties, Preprint 1998.

[Hun] B. Hunt, K3-fibred Calabi-Yau threefolds II, Singular fibers, Intern. J. Math. 10 (1999), pp. $871-896$.

[Hü] T. Hübsch, Calabi-Yau manifolds - A bestiary for physicists, World Scientific, 1991.

[H-L-Y] S. Hosono, B.H. Lian, and S.-T. Yau, Calabi-Yau varieties and pencils of K3 surfaces, alg-geom/9603020.

[H-S] B. Hunt and R. Schimmrigk, K3-fibred Calabi-Yau threefolds I, The twist map, Intern. J. Math. 10 (1999), pp. 833 - 869.

[Ka] T. Kaneyama, On equivariant vector bundles on an almost homogeneous varieties, Nagoya Math. J. vol 57 (1975), pp. 65 - 86.

[Ke] S. Keel, Toric varieties, course given at the Department of Mathematics, U.T. Austin, spring 1999; also private discussions.

[K-L-M] A. Klemm, W. Lerche, and P. Mayr, K3-fibrations and heterotic-type II string duality, Phys. Lett. $\mathbf{B 3 5 7}$ (1995), pp. 313 - 322.

[K-L-R-Y] A. Klemm, B.H. Lian, S.-S. Roan, and S.-T. Yau, Calabi-Yau fourfolds for M- and F-theory compactifications, Nucl. Phys. B518 (1998), pp. 515 - 574. 
[K-M] M. Kontsevich and Yu. Manin, Quantum cohomology of a product (with appendix by R. Kauffman, Invent. Math. 124 (1996), pp. 313 - 339.

[K-S1] M. Kreuzer and H. Skarke, Calabi-Yau 4-folds and toric fibrations, J. Geom. Phys. 26(1998), pp. 272 - 290.

[K-S2] _ Reflexive polyhedra, weights and toric Calabi-Yau fibrations, math.AG/0001106.

[K-S-Z1] M.M. Kapranov, B. Sturmfels, nad A.V. Zelevinsky, Quotients of toric varieties, Math. Ann. 290 (1991), pp. 643 - 655.

[K-S-Z2] — Chow polytopes and general resultants, Duke Math. J. 67 (1992), pp. 189 - 218.

[La] S. Lang, Elliptic functions, 2nd ed. GTM 112, Springer-Verlag, 1987.

[Lo] J.A. de Loera, Triangulations of polytopes and computational algebra, thesis, Applied Mathematics at Cornell University, 1995.

[Mo1] R. Morelli, A theory of polyhedra. Adv. Math. 97 (1993), pp. 1 - 73.

[Mo2] - The K-theory of a toric variety, Adv. Math. 100 (1993), pp. 154 - 182.

[M-F-K] D. Mumford, J. Fogarty, and F. Kirwan, Geometric invariant theory, Ergeb. Math. Grenz. 34, Springer-Verlag, 1994.

[M-S] D. Mumford and K. Suominen, Introduction to the theory of moduli, in Algebraic geometry, Oslo 1970, F. Oort ed., pp. 171 - 222, Wolters-Noordhoff Publ. Groningen, 1972.

[M-V] D. Morrison and C. Vafa, Compactifications of F-theory on Calabi-Yau threefolds: I, Nucl. Phys. B473 (1996), pp. 74 - 92; II, Nucl. Phys. B476 (1996), pp. 437 - 469.

[Od1] T. Oda, Lectures on torus embeddings and applications, Tata Inst. Fund. Res. Lect. Math. Phys. 57, Springer-Verlag, 1978.

[Od2] - Convex bodies and algebraic geometry - an introduction to the theory of toric varieties, Springer-Verlag, 1988.

[Og1] K. Oguiso, On algebraic fiber space structures on a Calabi-Yau 3-fold, International J. Math. 4 (1993), pp. 439 - 465.

[Og2] - On certain rigid fibered Calabi-Yau threefolds, Math. Z. 221 (1996), pp. 437 448.

[Og3] - Calabi-Yau threefolds of quasi-product type, Doc. Math. 1 (1996), pp. 417 - 447.

[Po] J. Polchinski, String theory, I: An introduction to the bosonic string, II: Superstring theory and beyond, Cambridge Univ. Press, 1998.

[P-S] E. Perevalov and H. Skarke, Enhanced gauge symmetry in type II and F-theory compactifications: Dynkin diagrams from polyhedra, Nucl. Phys. B505 (1997), pp. 679 700.

[Ra] G. Rejesh, Toric geometry and F-theory/heterotic duality in four dimensions, J. High Energy Phys. (1998), hep-th/9811240.

[Re] M. Reid, Decomposition of toric morphisms, in Arithmetic and geometry: Papers dedicated to I.R. shafarevich on the occasion of his sixtieth birthday, vol. II, Geometry, M. Artin and J. Tate eds., pp. 395 - 418, Prog. Math. 36, Birkhäuser, 1983.

[Va1] C. Vafa, Evidence for F-theory, Nucl. Phys. B469 (1996), pp. 403 - 418

[Va2] - Lectures on strings and dualities, hep-th/9702201.

[Va3] - Mathematical aspects of string theory, course given at the Department of Mathematics, Harvard University, fall 1999.

[Zi] G.M. Ziegler, Lectures on polytopes, GTM 152, Springer-Verlag, 1995. 
